Historic, Archive Document

Do not assume content reflects current scientific knowledge, policies, or practices. 



\section{WHOLESALE TRADE}

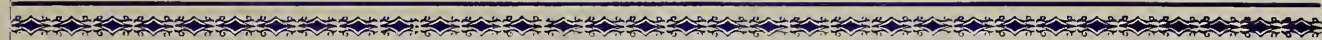
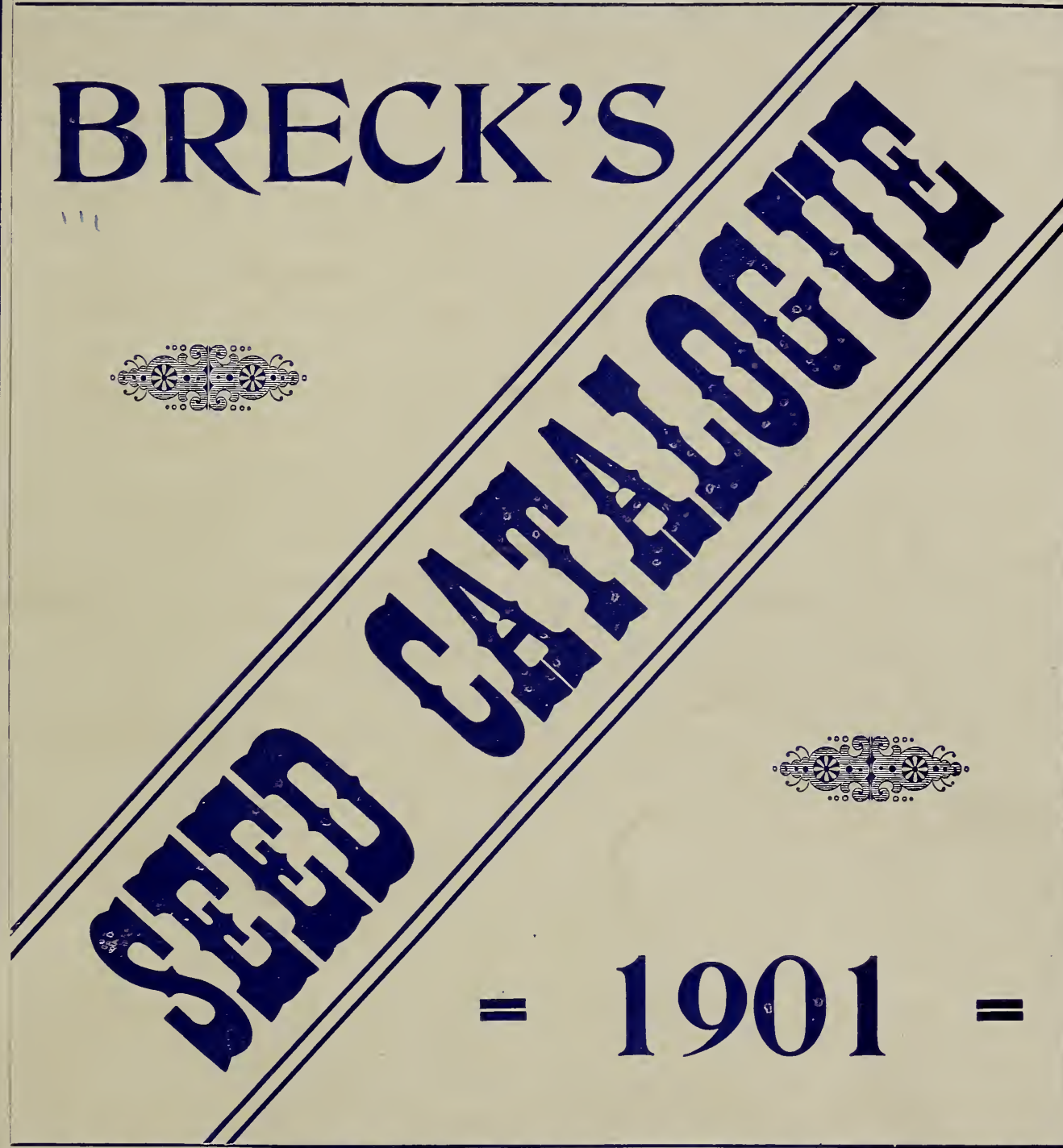

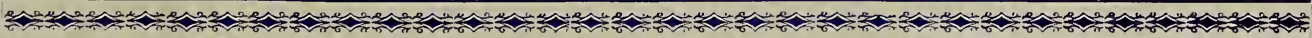

\section{Joseph Breck \& Sons (CORPORATION)}

47 to 54 NORTH MARKET STREET, BOSTON, MASS. 


\section{Code for Quantities.}

Quantity or number represented by the pound or bushel code word set opposite.

\begin{tabular}{|c|c|c|c|c|c|}
\hline $\begin{array}{l}\text { Quantity } \\
\text { or No. }\end{array}$ & $\begin{array}{c}\text { Pound } \\
\text { Code Word. }\end{array}$ & $\begin{array}{l}\text { Bushel } \\
\text { Code Word. }\end{array}$ & $\begin{array}{l}\text { Quantity } \\
\text { or No. }\end{array}$ & $\begin{array}{c}\text { Pound } \\
\text { Code Word. }\end{array}$ & $\begin{array}{l}\text { Bushel } \\
\text { Code Word. }\end{array}$ \\
\hline$\frac{1}{8}$ & Quanode & Quaphis & 28 & Quaoland & Quappend \\
\hline$\frac{1}{4}$ & Quanoint & Quaphony & 29 & Quaolast & Quappgar \\
\hline$\frac{1}{2}$ & Quanoka & Quaphote & 30 & Quaoler & Quappian \\
\hline$\frac{3}{4}$ & Quanold & Quapiary & 35 & Quaorcum & Quapsad \\
\hline 1 & Quanolis & Quapices & 40 & Quaostos & Quaptate \\
\hline 2 & Quanomia & Quapist & 45 & Quaouta & Quaqua \\
\hline 3 & Quanomy & Quapitz & 50 & Quapepsy & Quarado \\
\hline 4 & Quanon & Q sapium & 55 & Quaslade & Quattune \\
\hline 5 & Quanopsy & Quaplin & 60 & Quaspan & Quadit \\
\hline 6 & Quanotta & Quaplome & 65 & Quaspman & Quant \\
\hline 7 & Quanoura & Quapocan & 70 & Quassent & Quaurum \\
\hline 8 & Quanow & Quapoco & 75 & Quaston & Quaverse \\
\hline 9 & Quanrobe & Quapodal & 80 & Quaswoon & Quavel \\
\hline 10 & Quanrush : & Quapode & 85 & Quatar & Quavoke \\
\hline 11 & Quanser & Quapoets & 90 & Quatilt & Quavowed \\
\hline 12 & Quanside & Quapogee & 95 & Quatomy & Quawful \\
\hline 13 & Quansing & Quapogon & 100 & Quatrium & Quaxil \\
\hline 14 & Quanspet & Quapollo & 200 & Quatrous & Quaxilla \\
\hline 15 & Quanta & Quapolus & 300 & Quattar & Quazunge \\
\hline 16 & Quanteal & Quapopga & 400 & Quattain & Quaxiom \\
\hline 17 & Quantelo & Quaporio & 500 & Quattask & Qubaal \\
\hline 18 & Quantes & Quaporon & 600 & Quattent & Qubabble \\
\hline 19 & Quanther & Quapotol & 700 & Quatter & Qubabel \\
\hline 20 & Quantic & Quapozem & 800 & Quattest & Qubabery \\
\hline 21 & Quantler & Quappair & 900 & Quattic & Qubabish \\
\hline 22 & Quanvil & Quappal & 1000 & Quattire & Qubablah \\
\hline 23 & Quanwold & Quappavi & 2000 & Quattive & Qubaboon \\
\hline 24 & Quaobald & Quappay & 3000 & Quattorn & Qubacca \\
\hline 25 & Quaocus & Quappeal & 4000 & Quattrap & Qubacorn \\
\hline 26 & Quaoglus & Quappedo & 5000 & Qubasand & Qubasyle \\
\hline 27 & Quaogul & Quappelt & & & \\
\hline
\end{tabular}

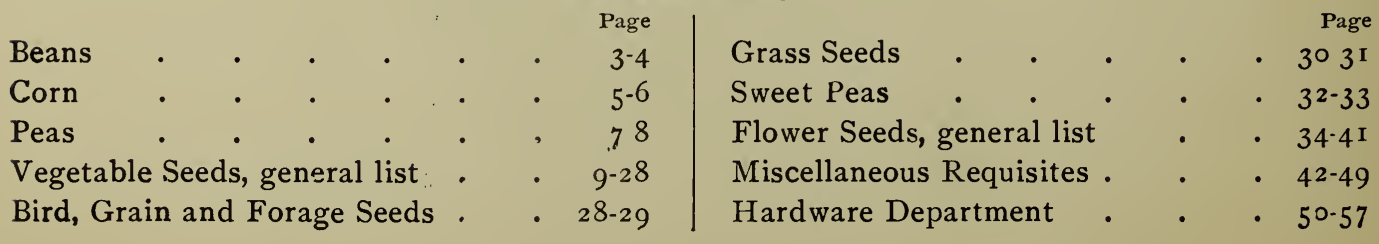




\section{Wholesale Price List}

. ๑ ๑F ...

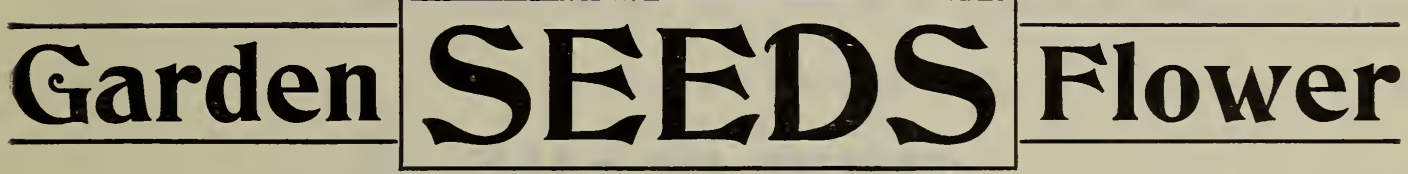

Offered by

\section{JOSEPH BRECK \& SONS \\ (Corporation)}

SEED GROWERS AND WHOLESALE MERCHANTS

47 to 54 North Market Street, 


\section{Wholesale Trade List.}

It is generally known that unfavorable conditions at home and abroad during the growing and harvesting season, have seriously reduced the supply of many varieties of seeds, and that prices as a result are somewhat higher. We believe the actual shortage is not yet realized, and that there will be a seed famine before the end of the planting season of rgor. We therefore recommend early buying and the obtaining of better profits at retail than have prevailed for some years.

We also wish to state that while our stocks are limited, we have on hand at present a fair supply of most kinds of Beans, Peas, Corn and other vegetable seeds, and will promptly fill, at reasonable market prices, all orders sent us so long as supplies permit.

\section{TERMS OF SALE.}

VEGETABLE AND FLOWER SEEDS. - Net cash, June I. A discount of I $\%$ per month for unexpired time allowed for cash.

FIELD PEAS, GRAINS, GRASS, BIRD and other MISCELLANEOUS SEEDS. Net cash 30 days, without any discount whatever. All bills not paid at maturity are subject to sight draft.

Remittances must be made direct to our Boston office.

The prices named are F. O. B., Boston, and are subject to change without notice. We do not bind ourselves to quantity. Orders will be filled at lowest prices the market warrants at date of shipment. Firm quotations made when desired.

All claims for shortage, errors or overcharge, must be made within five days after receipt of goods.

NOTICE. - While we exercise the greatest care to have all Seeds, Plants, Bulbs and Nursery Stock of the best kind, we do not give, and our agents and employees are forbidden to give any warranty, express or implied. If you do not accept these goods on these terms, you must return them at once, and all payments therefor will be refunded.

\section{JOSEPH BRECK \& SONS, (CORPORATION.)}

The Telegraphic Cipher attached to each variety, if used in connection with the code for quantities, will enable customers to wire us orders at small expense. Prefix the word, Freight, Express or Mail, to designate the manner of shipment desired. 'The code word for quantities must always precede the words for seeds, although if the same quantity of several varieties is wanted, the quantity word need only be placed before the word for the first article ordered. For Example, "Freight Quapode, Blacklink, Baker, Beauty, Quanopsy, Brabast, Capture, Claubble," means, "Send immediately by first freight ten bushels each Farly Carmine Podded Horticultural, Early Round Yellow Six Weeks and Yellow Cranberry Dwarf Beans, also five pounds each Arlington Early Turnip Blood Beet, All Season's Cabbage, and Early Half Long Scarlet Carrot." “Express Quapepsy, Brew," means, "Send inmediately by express fifty pounds Improved Mammoth Long Red Mangel Wurzel." "Mail Quanomia, Chemic," means, "Send by first mail post paid, two pounds Premium Late Flat Dutch Cabbage."

NOTE. - Code used in this list is adopted by the American Seed Trade Association. 
Beans, Corn and Peas, pages 7 to 12. Alphabetical List begins page 12.

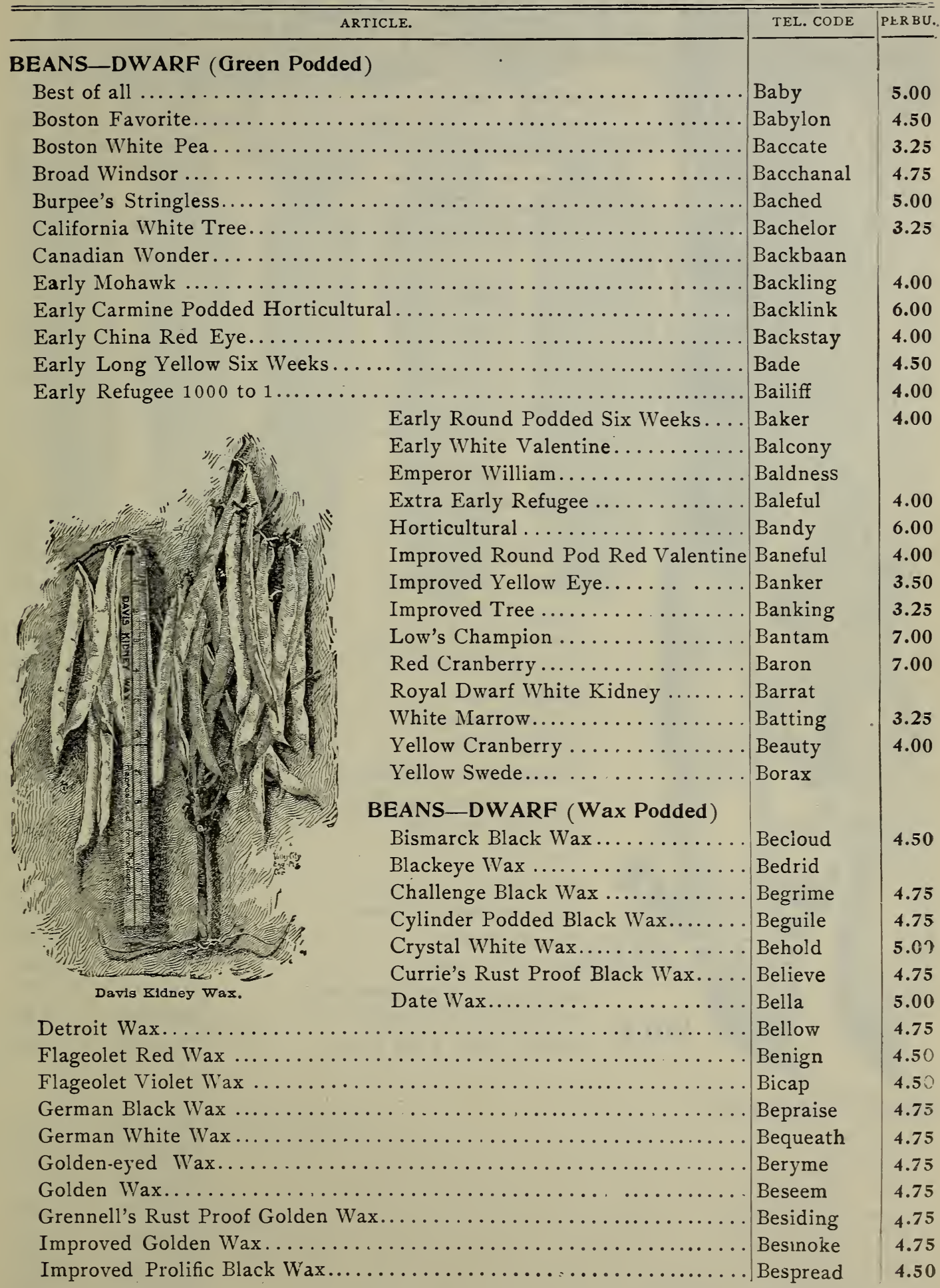




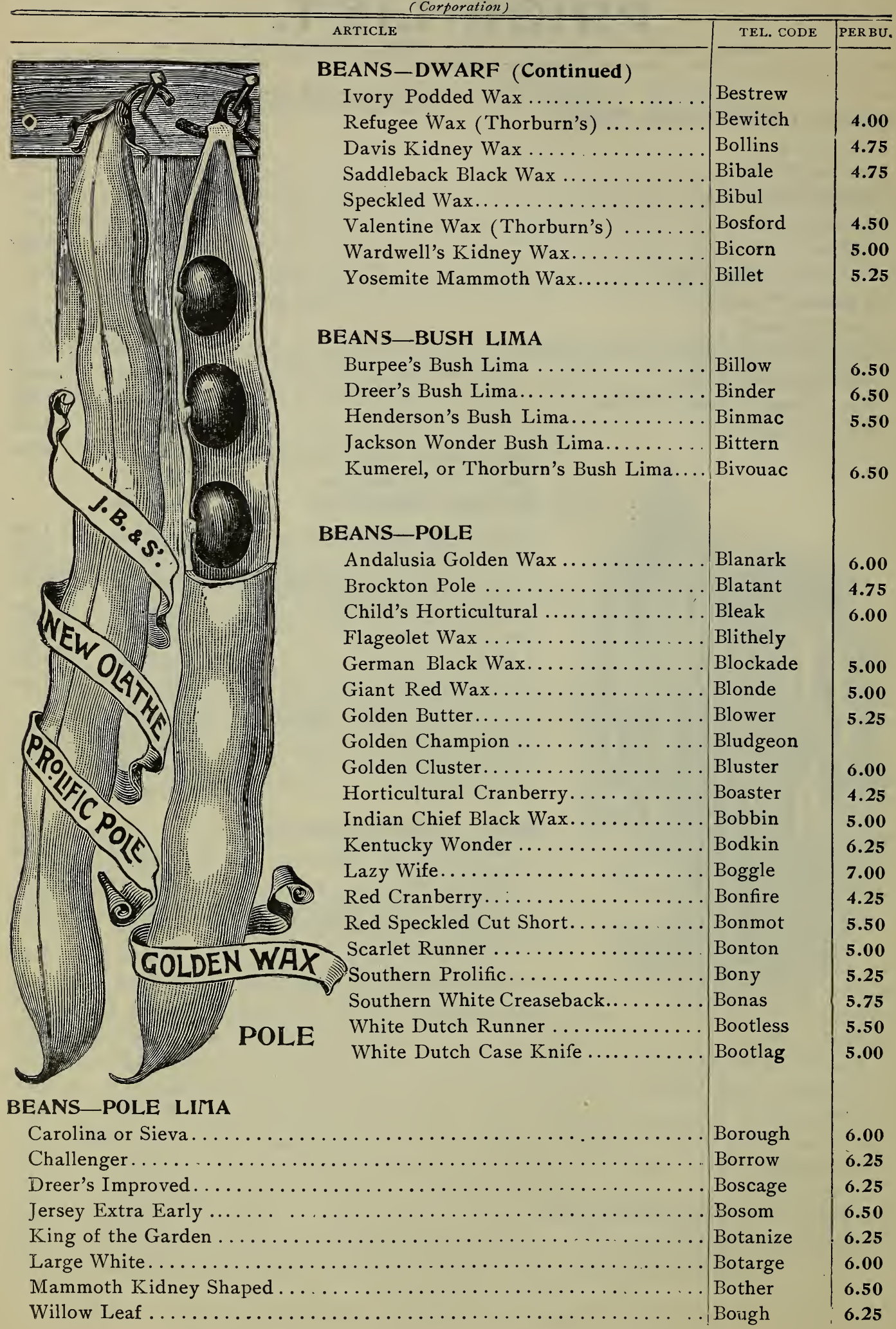




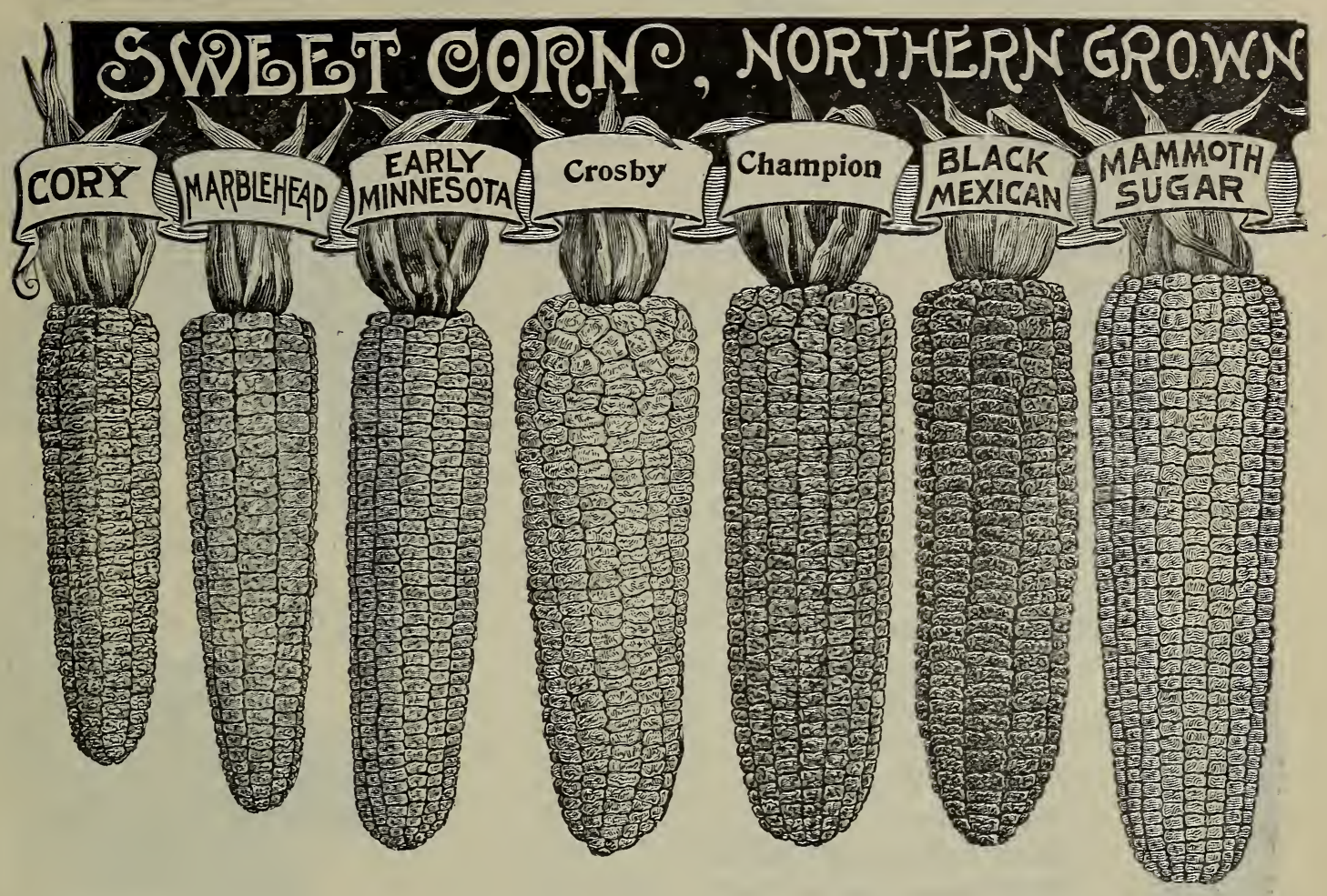

\begin{tabular}{|c|c|c|}
\hline ARTICLE & TEL. CODE & PER BU. \\
\hline Adams' Early. & Confab & 1.75 \\
\hline Adams' Extra Early. & Confect & 1.80 \\
\hline$\ldots \ldots \ldots \ldots \ldots \ldots \ldots \ldots$ & Confection & 2.25 \\
\hline$\ldots \ldots \ldots \ldots \ldots \ldots \ldots \ldots \ldots \ldots \ldots \ldots \ldots \ldots \ldots \ldots$ & Confeder & 2.25 \\
\hline$\ldots \ldots \ldots$ & Confer & 2.25 \\
\hline$\ldots \ldots$ & ence & 2.50 \\
\hline $\mathrm{Br}$ & $\mathrm{Co}$ & 2.50 \\
\hline$\ldots \ldots \ldots \ldots \ldots \ldots \ldots$ & & 2.50 \\
\hline $\mathrm{Bu}$ & Cor & 2.25 \\
\hline$\ldots \ldots \ldots$ & & 2.50 \\
\hline 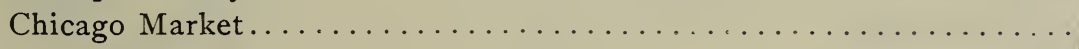 & Co & 2.25 \\
\hline 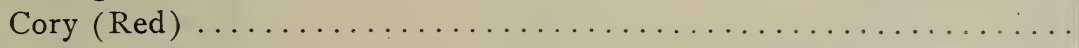 & & 2.25 \\
\hline $\mathrm{Co}$ & $\mathrm{Co}$ & 2.25 \\
\hline . & ent & 2.25 \\
\hline $\mathrm{Ea}$ & $\mathrm{Co}$ & 2.25 \\
\hline & & 2.25 \\
\hline gton Market....... & Co & 2.00 \\
\hline vergreen & & 2.50 \\
\hline Go & Co & 2.50 \\
\hline $\mathrm{Hi}$ & Co & 2.25 \\
\hline & $\tan t$ & 2.25 \\
\hline te Cory. . & & 2.75 \\
\hline & & 2.00 \\
\hline $\mathrm{F}$ & & 2.25 \\
\hline Old Colony ....... & Continent & 2.25 \\
\hline
\end{tabular}




\begin{tabular}{|c|c|c|c|}
\hline \multicolumn{2}{|r|}{ ARTICLE } & TEL. CODE & PER BU. \\
\hline \multicolumn{4}{|c|}{ CORN_SWEET (Continued) } \\
\hline \multirow{2}{*}{\multicolumn{2}{|c|}{ 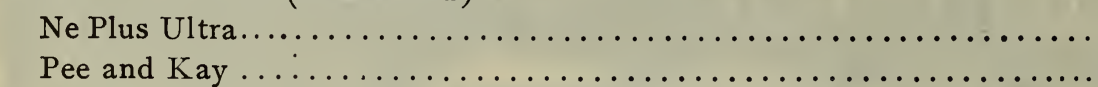 }} & Contagion & 2.25 \\
\hline & & Contour & 2.25 \\
\hline \multicolumn{2}{|c|}{ 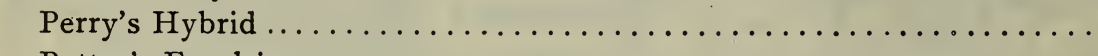 } & Cont & 2.25 \\
\hline \multicolumn{2}{|c|}{ 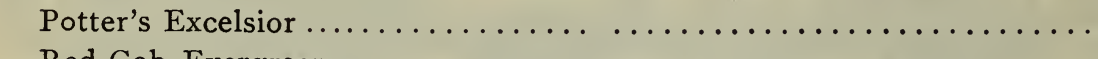 } & Contribe & 2.25 \\
\hline \multicolumn{2}{|c|}{ 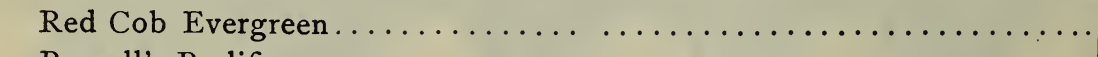 } & Cor & 2.25 \\
\hline \multicolumn{2}{|c|}{ 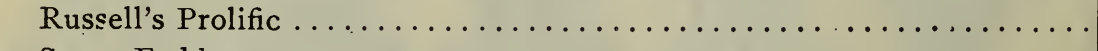 } & Conv & 2.25 \\
\hline \multicolumn{2}{|c|}{ 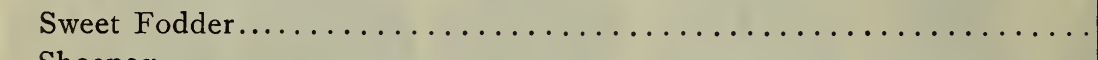 } & Co & 2.25 \\
\hline \multicolumn{2}{|r|}{ 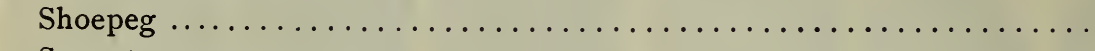 } & $\mathrm{Co}$ & 1.10 \\
\hline \multicolumn{2}{|c|}{ 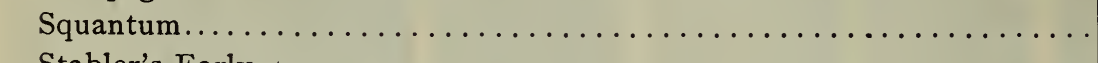 } & Cor & 2.25 \\
\hline \multicolumn{2}{|c|}{ 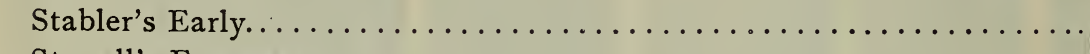 } & $\mathrm{Cos}$ & 2.25 \\
\hline \multicolumn{2}{|c|}{ 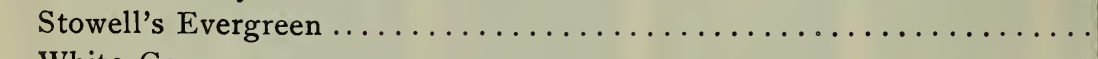 } & Cooler & 2.00 \\
\hline \multicolumn{2}{|r|}{ ( } & $\mathrm{Co}$ & 2.50 \\
\hline \multicolumn{2}{|c|}{ 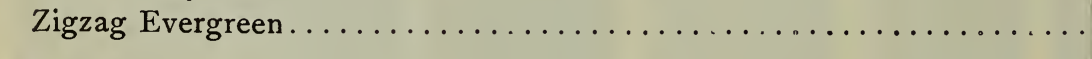 } & Copious & 2.75 \\
\hline \multicolumn{4}{|c|}{ CORN-FIELD } \\
\hline (20) & Angel of Midnight . & Coppice & 1.25 \\
\hline & t's $\mathrm{P}$ & Cop & 1.00 \\
\hline & $\ldots \ldots \ldots \ldots \ldots \ldots$ & $\mathrm{Coq}$ & 1.25 \\
\hline 23 & $\mathrm{Pe}$ & Cor & 1.00 \\
\hline Sen & $\mathrm{Da}$ & $\mathrm{Co}$ & 1.00 \\
\hline (6) & Eight-rowed C & Cor & 1.25 \\
\hline & Early Huron Yellow Dent ..... & Cornet & 1.00 \\
\hline this & $\mathrm{Ea}$ & Cor & 1.00 \\
\hline Sy & Wisconsin Yellow Dent.... & Corrag & 1.00 \\
\hline (2) & 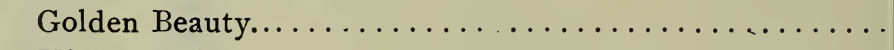 & $\mathrm{Co}$ & .90 \\
\hline 5 & $\mathrm{Hic}$ & Cosmic & 1.00 \\
\hline 3 & $\operatorname{ming} \ldots \ldots \ldots \ldots$ & $\mathrm{Co}$ & .90 \\
\hline 30 & Iowa Gold M & Costape & 1.00 \\
\hline 13: & 然 & $\operatorname{Cos} t$ & .90 \\
\hline 35 & ellow Flint............ & Cou & 1.00 \\
\hline 85 & $\mathrm{Mi}$ & Co & 1.00 \\
\hline & 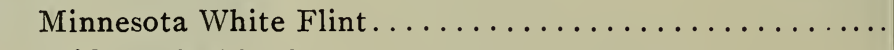 & Cor & 1.00 \\
\hline Sto & Torth...... & Cousin & .90 \\
\hline -1) & Cob En & Cover & .85 \\
\hline all & Riley's Favo & Covertly & .90 \\
\hline & Sanford, or Long White & Covey & .90 \\
\hline & Sheep's Tooth Ensilage........ & Coward & .85 \\
\hline & St. Charles' White............ & Cowhonk & .80 \\
\hline & W & Cowma & .80 \\
\hline 39 & ite Flint & Cowm & .80 \\
\hline & Wisconsin Yellow Dent... & Cowmug & .90 \\
\hline $\mathrm{RN}-\mathrm{P}$ & & & PER LE \\
\hline & & & .05 \\
\hline White & & Craunch & .05 \\
\hline Pearl. & & & .05 \\
\hline Red I & & Crawl & .06 \\
\hline
\end{tabular}




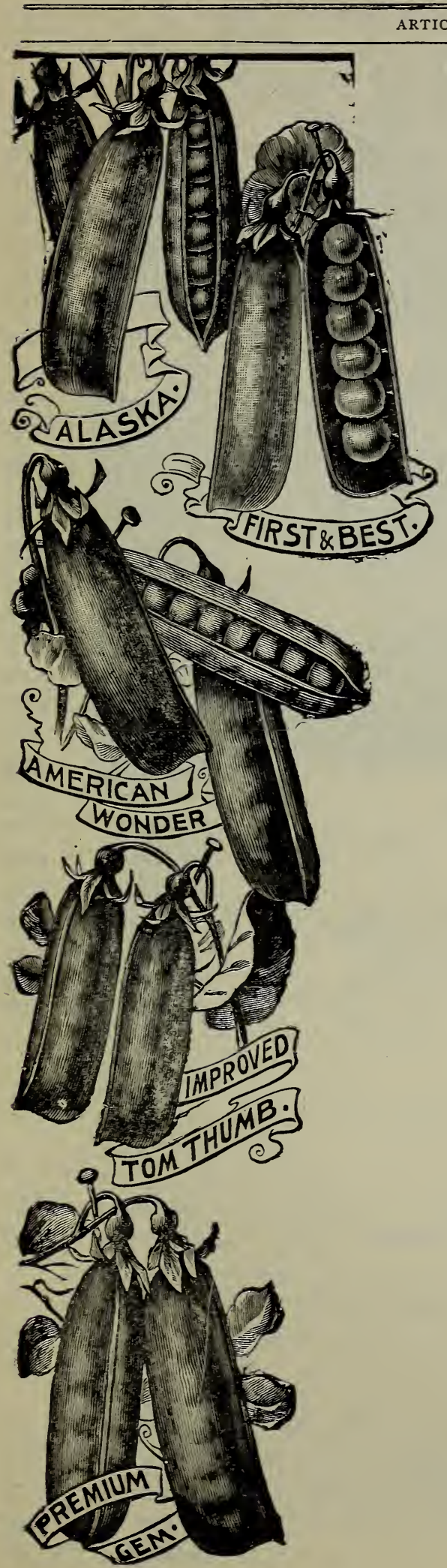

\section{PEAS}

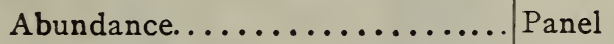

Admiral................... Panic

3.25

Alpha ................. Panoply

2.75

American Champion .......... Panorama

3.25

American Wonder............ Pantaloon

3.75

Bergen Fleetwing.............. Pantofle

5.50

Blackeyed Marrowfat .......... Pantomime

3.25

Blue Beauty................ Papacy

Blue Imperial. . . . . . . . . . Papal

1.75

3.50

Blue Peter................ Papist

3.00

Blue Perfection.............. Paper

3.50

Boston Extra Early........... Parable

3.50

Breck's Excelsior............. Parade

Breck's New Life............ Paradigm

3.25

3.25

British Queen............. Paradise

4.00

Burpee's Quantity........... Parallox

3.25

4.00

Canada Field, Blue Prussian $\mathscr{E}_{0}$. Parallel

1.50

Canada Field, Golden Vine Paramount

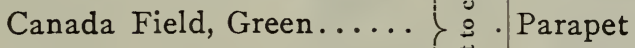

1.25

Canada Field, Scotch ..... Paraquet

1.50

Canada Field, White......

1.50

Caractacus................ Parasol

Carter's First Crop............ Parcel

Carter's Premium Gem......... Parener

Carter's Stratagem ........... Parchment

1.20

3.25

3.25

4.25

4.00

Carter's Telephone........... Pardon

Champion of England......... Parent

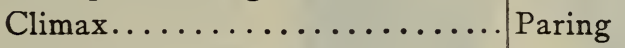

Culverwell's Telegraph........ Parish

Daniel O'Rourke ............. Parlance

Dwarf Champion............ Parol

Dwarf White Marrowfat ....... Paroling

Duke of Albany ............ Parotid

Dwarf Telephone ............ Phonedwf.

4.00

3.00

3.25

3.50

3.25

4.15

1.75

4.15

Daisy ................... Paisyd

Early Frame or May .......... Parsmony

Early Kent ............... Parsonage

Everbearing ............... Partisan

Extra Early Alaskas........... Parschal

Kentish Invicta............ Passenger

Maud " $\mathrm{S}$ "................ Passion

Tom Thumb............... Pastime

First and Best.............. Pasturage

Field Southern Blackeyed Cow ... Patrol

Field Whip-poor-will.......... Patron

French Canner............. Patty

Gradus (New).............. Pattern

Hancock.............. Pavement

5.25

5.25

3.25

3.25

3.25

3.75

3.25

3.25

3.50

3.40

2.25

2.25

2.25 


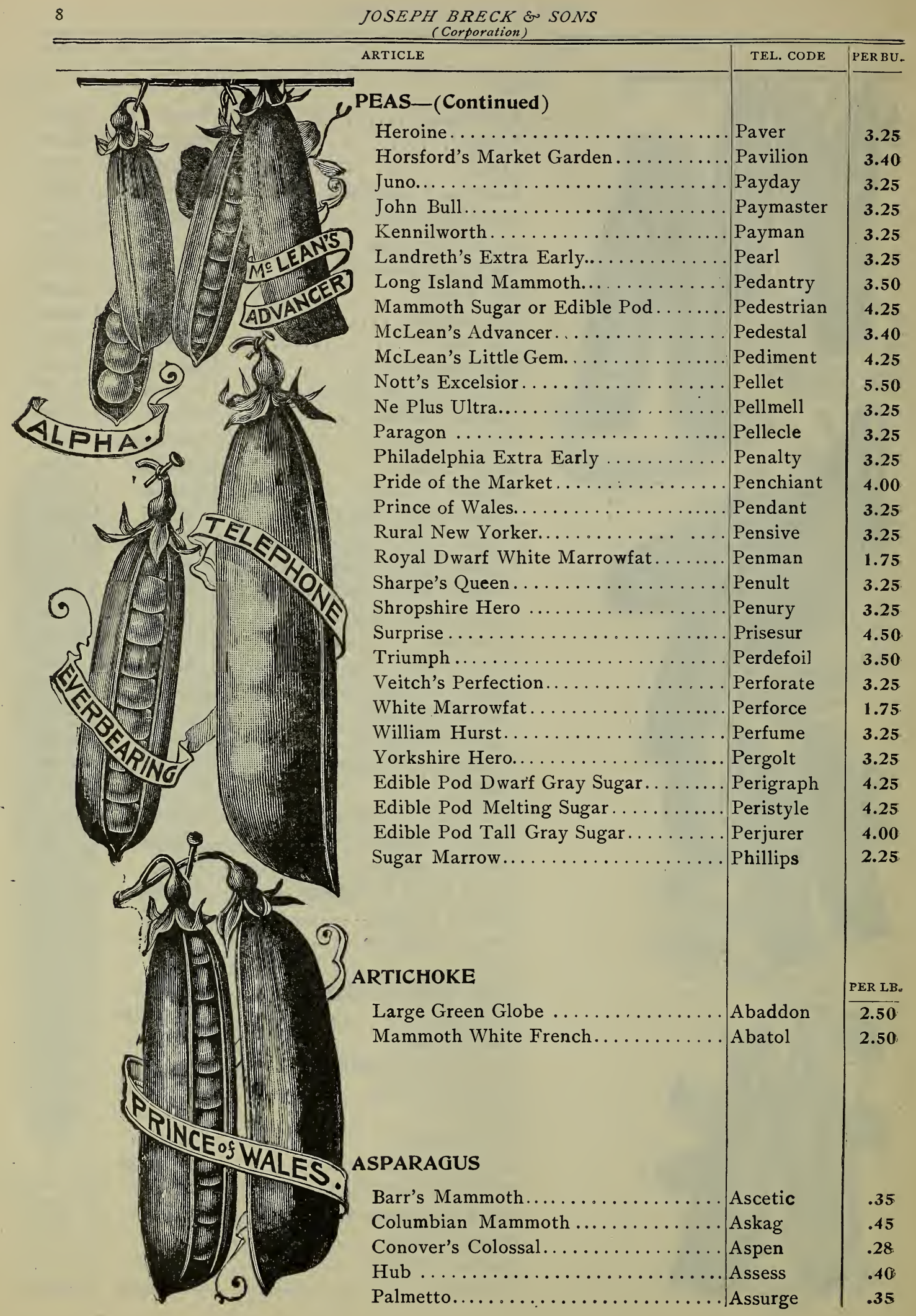




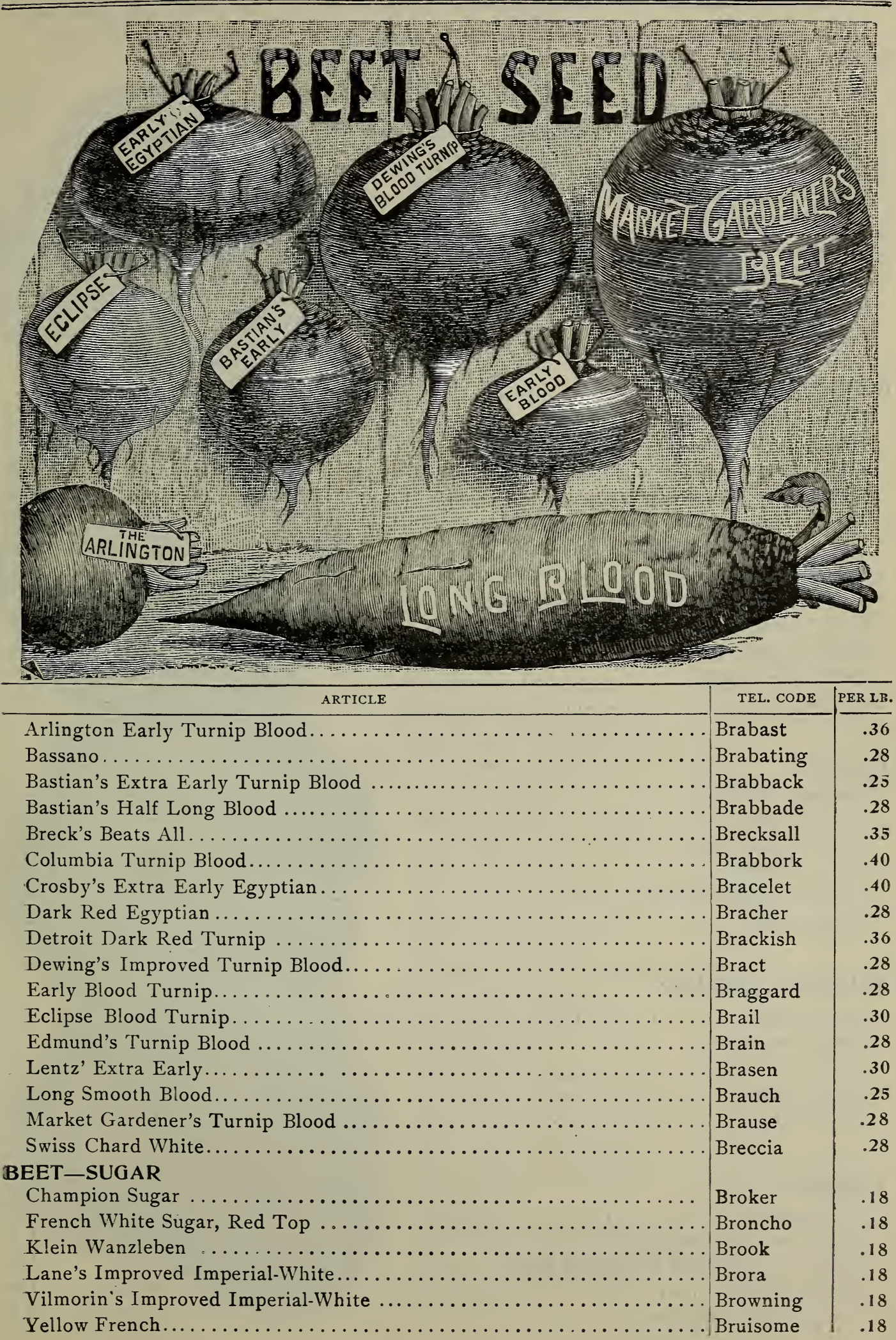




\section{BEET-MANGEL WURZEL}

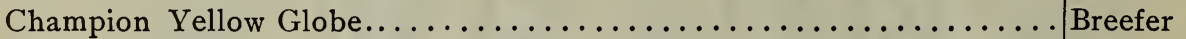

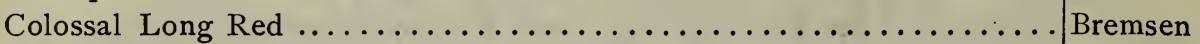

Dignity

ARTICLE

\begin{tabular}{l|r}
\multicolumn{1}{c|}{ TEL. CODE } & PERT. \\
\cline { 1 - 2 } & \\
Breefer & .18 \\
Bremsen & .18 \\
Brenker & .18 \\
Brennage & .18 \\
Breve & .18 \\
Brevier & .18 \\
Brew & .18 \\
Brilliant & .18 \\
Brindle & .18 \\
Brisk & .18 \\
&
\end{tabular}

\section{BORECOLE OR KALE}

Brown German Curled

Dwarf Curled Scotch

Dwarf Green Curled.

Dwarf Green Scotch.

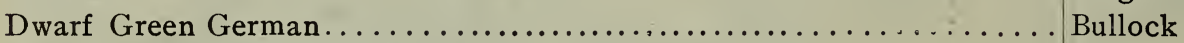

Dwarf Green Curled Erfurt ........................ Bully

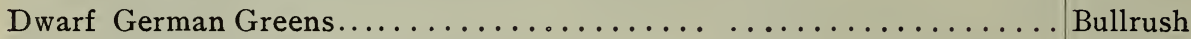

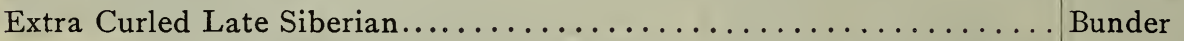

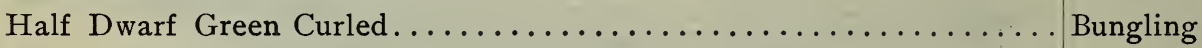

Moss Curled.

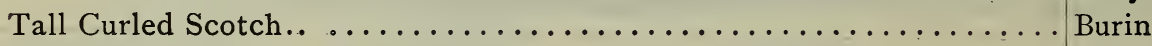

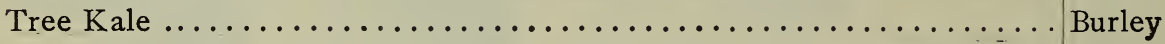

\section{BROCOLI}

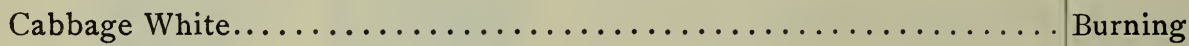

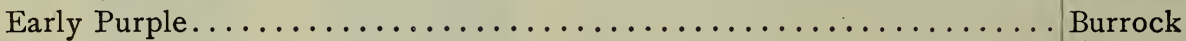

Early Purple Cape $\ldots \ldots \ldots \ldots \ldots \ldots \ldots \ldots \ldots \ldots \ldots \ldots \ldots$ Bursche

3.00

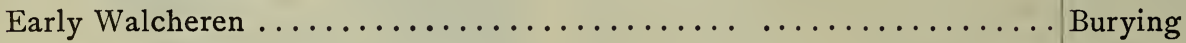

3.00

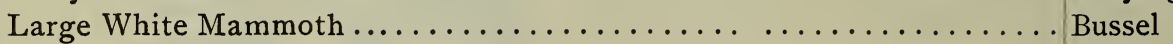

3.50

3.00

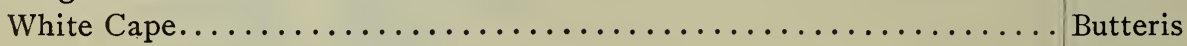

\section{BRUSSELS SPROUTS}

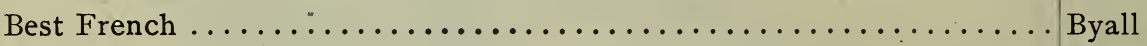

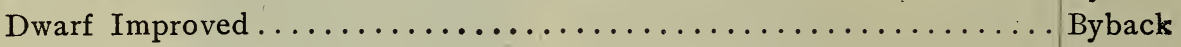

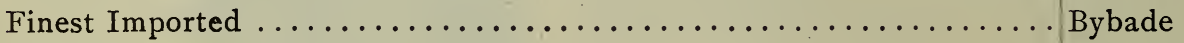

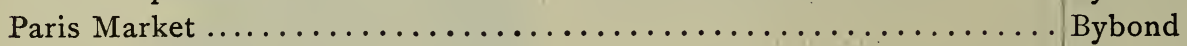

Tall French $\ldots \ldots \ldots \ldots \ldots \ldots \ldots \ldots \ldots \ldots \ldots \ldots \ldots \ldots$ Byway

\section{CABBAGE}

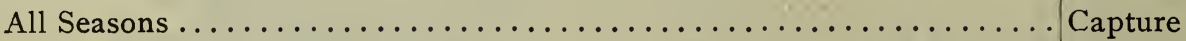

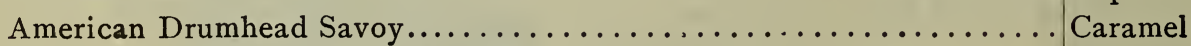

American Perfection Drumhead Savoy.................... Carane 


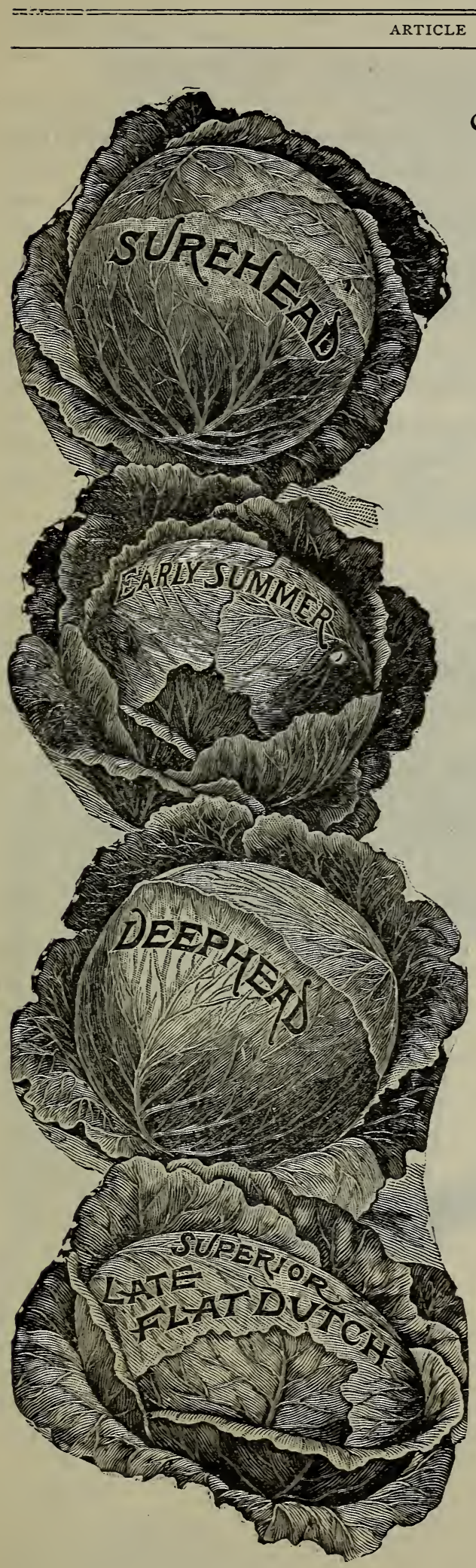

\section{CABBAGE - (Continued)}

Bridgeport Drumhead....... Caravan

Burpee's All Head, Early .... . Caravas

Charleston Large Wakefield ... Carbine

Chase's Excelsior........... Carbisk

Curled Savoy............ Carcan

Danish Ball Head.......... Carcass

Danish Round Winter....... Cardham

Dark Red Dutch .......... Carder

Deephead............. Cardin

Earliest Etampes ....... . . Carnal

Early Drumhead .......... Carpen

1.25

1.50

1.85

Early Dwarf Flat Dutch...... Carrom

Early French Oxheart........ Carte

Early French Jersey Wakefield Carun

EarlyFrench Newark FlatDutch Cascade

Early French Oxheart....... Cascas

Early French Red Drumhead.. Casque

Early French Winningstadt.... Castel

Early French York......... Cathol

Extra Early Express ......... Causer

Faultless............... Cavern

Filderkraut ............. Cavil

Fottler's Early Brunswick .... Cavity

Henderson's Early Summer.... Centime

Henderson's Succession ...... Cento

Holland ............... Central

Hundredweight............ Centric

Large Early Schweinfurt..... . Chalice

Large Early York ........... Chamade

1.85

1.25

1.75

.90

1.00

1.10

1.00

2.00

1.40

1.00

2.00

.85

.80

1.00

1.00

.90

1.10

1.50

1.60

1.85

1.25

1.00

Large Late Drumhead ....... Chancel

.80

1.10

Large Late Flat Dutch....... Chandler

Louisville Drumhead........ Chare

Luxemburg Hard Heading .... Charade

1.00

1.20

Mammoth Rock Red ........ Chariot

1.25

Marblehead Mammoth....... Charity

Peerless Early .......... Checker

Premium Drumhead......... Cheer

Premium Late Flat Dutch .... Chemic

Short Stem Drumhead ....... Childish

2.00

1.25

1.00

1.25

1.10

Stein's Early Flat Dutch ...... Chime

1.40

1.50

Stone Mason Drumhead ...... Chimney

Surehead ............ Chintz

1.25

Vandergaw's Flat Dutch..... Chip

Warren's Improved Drumhead Chival

1.50

1.50

Washington's Wakefield ...... Chock 


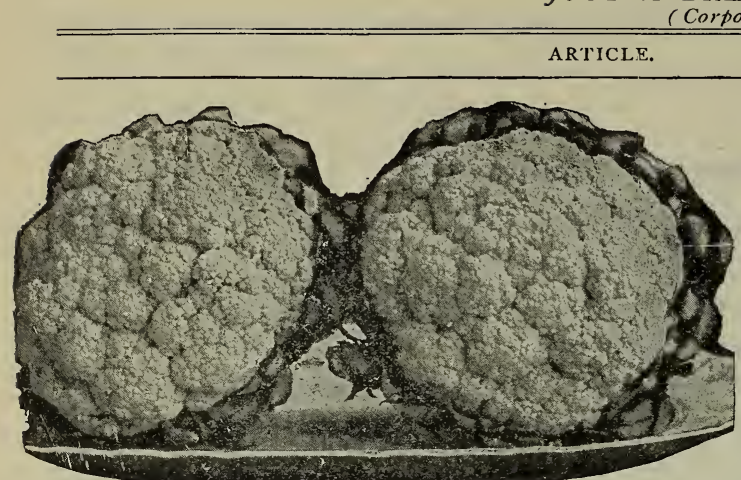

Snowball Oauliflowers.
CAULIFLOWER

Autumn Giant......... Cibank

Breck's White Bouquet ... Cidold

Early Dwarf Erfurt ..... Cibospen

Eariy Dwarf Favorite.... Cibostark

Early Dwarf London.... . Cibotog

Early Dwarf Walcheren ... Cibottom

Extra Early Paris ........ Cicaque

Extra SelectedDwarf Erfurt Cicake

Half Early Paris........ Cicalve

Henderson's Snowball.... Cicame

Cicamord

Large Late Algiers

Le Normand's Short Stem

New Model

CELERY

Boston Market ........

Carter's Dwarf Crimson.

Covent Garden Rose....

Crawford's Half Dwarf..

Dwarf White Solid......

Early Arlington . . . . . . .

Giant Pascal...........

Giant White Solid.......

Golden Heart .........

Golden Self Blanching.

Heartwell Perfection....

Henderson's Half Dwarf

Henderson's Pink Plume

Imcomparable Dwarf

Crimson ...........

Improved White Plume..

Kalamazoo ............

New Rose ............

Solid Ivory...........

Soup Celery..........

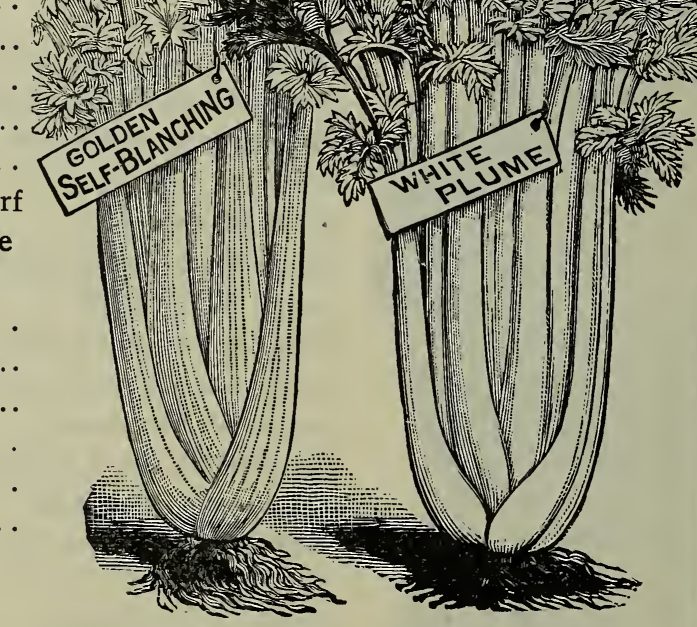

Cidade

Cidaling

Cobble

Cobweb

Cocket

Cockney

Codlin

Codload

Cohert

Coif

Coinage

Comely

Collab

Collabing

College

Collier

Collude

Colly

Comer

Compile

Complex

1.25

1.00

1.00

1.25

1.10

1.00

1.25

1.10

1.50

2.50

1.25

1.25

1.50

1.00

1.50

1.25

1.50

1.10

.12

1.25

1.25

1.25

1.25

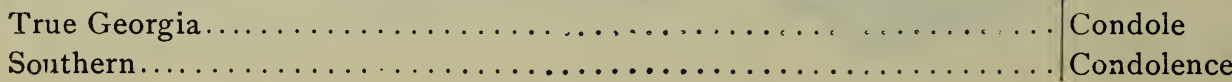




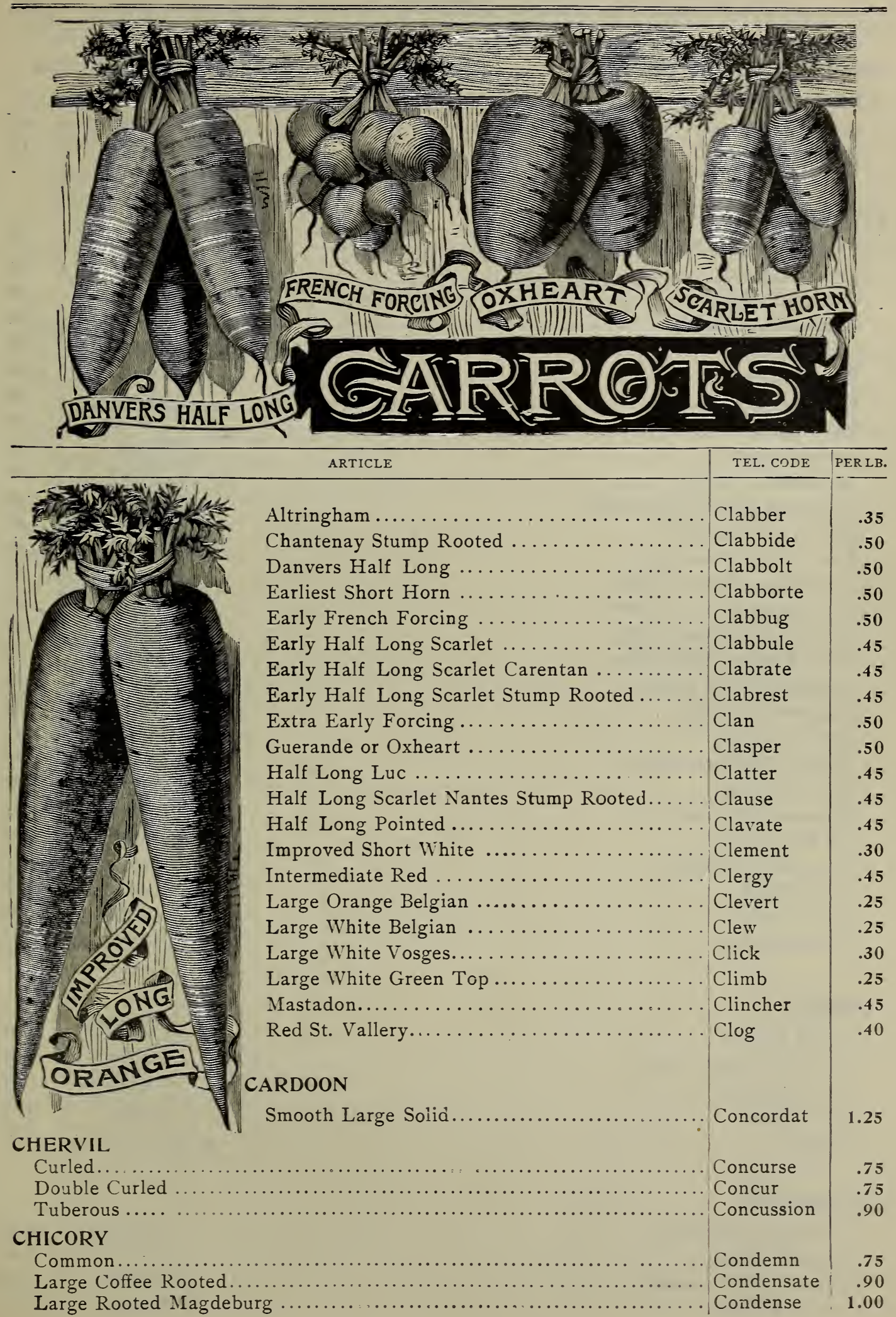




\section{CORN SALAD OR FETTICUS}

Broad Leaved

Large Seeded

Condone

Small Seeded

\section{CUCUMBER}

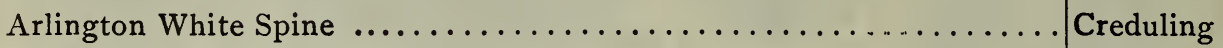

Boston Pickling .

Chicago Pickling

Cremona

Commercial Pickling.

Cool and Crisp

Early Cluster.

Early Frame.

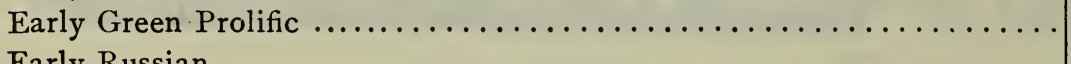

Early Russian

Early Short Green

Crewel

Evergreen Early White Spine

Cribble

Extra Early Siberian

Crimp

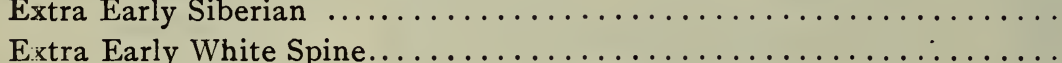

Extra Long Green Prickly.

Cringle

Crinkle

Extra Long Green Smooth

Crinoline

Cripple

Extra Long Green Turkey.

Crisis

Hill's Forcing White Spine

Crocket

Improved White Spine. . .

Crosier

Japanese Climbing

Cross

Jersey Pickling

Crossbol

Nichol's Medium Green

Crucial

Paris Pickling

Crucifix

Peerless White Spine.

Tailby's Hybrid. .

Crural

West India Gherkin

Crust

White Dutch.

White Pearl .

Crustily

Crutch

White Wonder

Crystal

\section{CRESS}

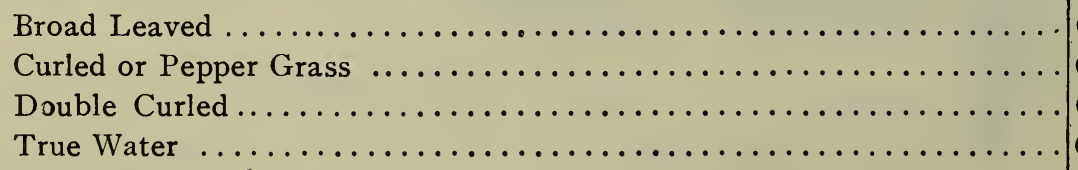

Cumulate

Upland

\section{DANDELION}

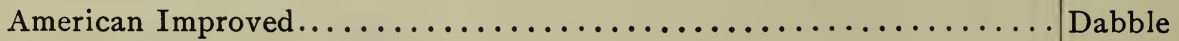

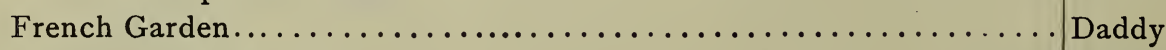

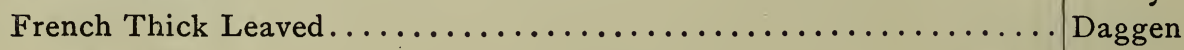




$\overline{\text { ARTICLE }}$

EGG PLANT

Black Pekin

Early Dwarf Purple.

Eager

Eagle

Early Long Purple

Earlap

1.25

Improved Large Purple.

Earing

Easter

Long White

Eccentric

New York Improved

Eclipsing

Pearl

Eden

2.00

2.75

2.50

2.75

3.75

\section{ENDIVE}

Broad Leaved Batavian

Early White Curled...

Fine Moss Curled

Egotist

Eldert

Elephant

Green Broad Leaved

Ember

Large Green Curled.

Emergent

Small Green Curled .

Emulsion

White Curled.

Enactor

\section{GOURDS}

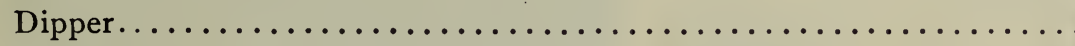

Dishcloth

Galoche

Gambit

Hercules Club

Garmerk

Japanese Nest Egg

Gartol

Japanese Climbing

Gasket

\section{HERBS}

Angelica

Anise

Haberd

Habitat

Balm

Hackle

Basil, Sweet.

Haggard

Belladonna

Halberd

Borage.

Caraway.

Halser

Catnip Halter

Coriander Hamlet

Dill Hamper Harbinger

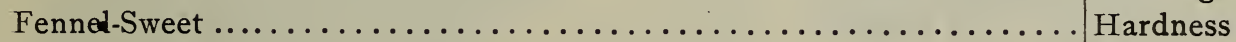

Hop Hauteur

Horehound Haven

Hyssop Havoc

Lavender Hawser

Marjoram, Sweet. Heartburn

Pennyroyal. Hedgerow

Rue.. Heirloom

Rosemary Helical

Saffron 


ARTICLE

\begin{tabular}{l|l}
\multicolumn{1}{c|}{ TEL. CODE } & PER LB \\
\cline { 1 - 2 } & \\
Hercules & $\mathbf{3 . 0 0}$ \\
Hermit & $\mathbf{2 . 0 0}$ \\
Hernia & $\mathbf{2 . 2 5}$ \\
Hiatus & 2.00 \\
& \\
& \\
Keblap & $\mathbf{1 . 7 5}$ \\
Kolpie & $\mathbf{1 . 5 0}$ \\
Kerchief & $\mathbf{1 . 2 5}$ \\
Kildong & $\mathbf{1 . 7 5}$ \\
Knave & $\mathbf{1 . 0 0}$
\end{tabular}

\section{LEEK}

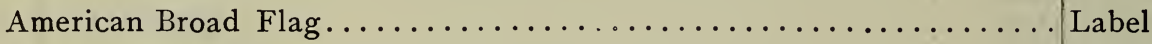

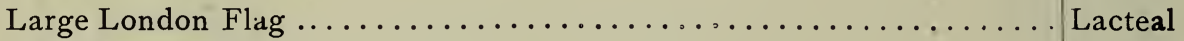

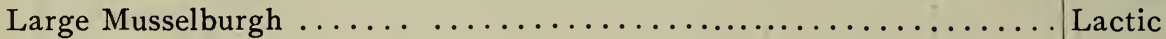

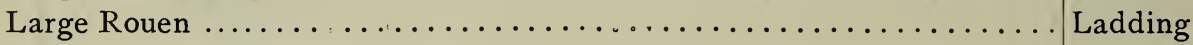

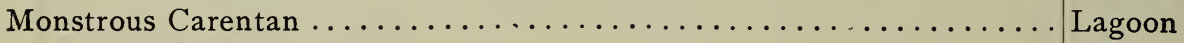
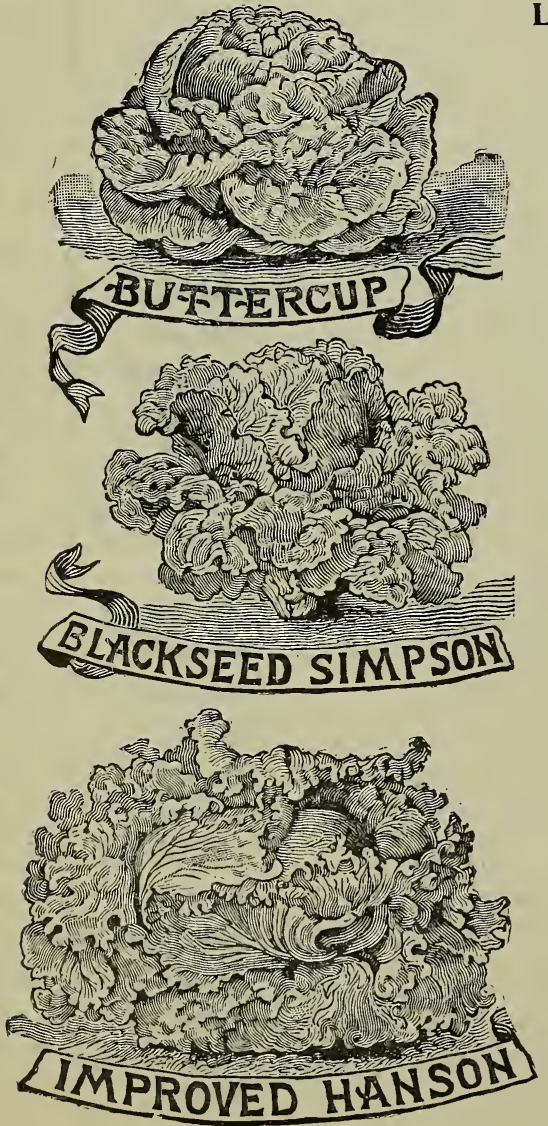

\section{LETTUCE}

All the Year Round............ Lanby

Arlington Tennisball........... Lancard

American Gathering........... Lancer

Big Boston............... Lanchup

Black Seeded Butter........... Landlock

Black Seeded Satisfaction........ Landlord

Blond Blockhead............. Landscape

Boston Curled ............... Landslide

Boston Market.............. Langel

Brown Dutch ............... Lanking

Buttercup ................ Lapidist

California Cream Butter......... Lapstone

Chartier or Brown Curled......... Lares

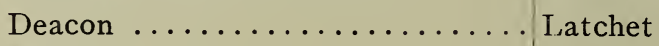

Defiance ................. Lather

Denver Market ............. Lattice

Drumhead or Malta ........... Laudable

Early Curled Silesia............ Laughter

Early Curled Black Seeded Simpson Laureate

Early Curled White Seeded Simpson Laxity

Early Prizehead............. Layman

Early White Cabbage or Butter..... Lazar

Frankfurt Head, Black Seeded ..... Leaven

Golden Ball ............... Ledger

Golden Stonehead ........... Legbail

Grand Rapids Forcing.......... Leggin

Green Fringed.............. Legion
.60

2.00

.55

1.00

.50

.60

.70 .

.75

.60

.60

.60

.70

.60

.60

.75

.60

.50

.50

.60

.55

.55

.60

.60

.60

.60

.60

.60 


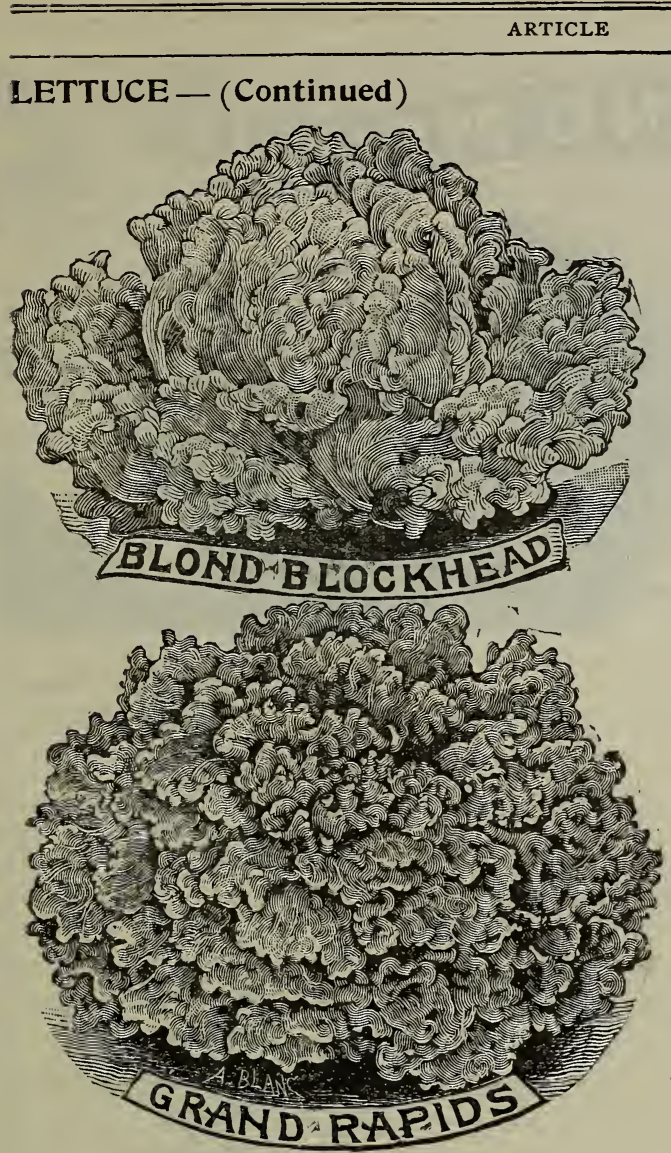

Hardy Green Winter ...... Lengthen Henderson's New York ..... Lengwood Hubbard's Market ......... Lessing Iceberg ............... Lesson Improved Hanson ......... Levant Marblehead Mammoth ..... Lighter Oak Leaved ............. Limpet Paris Green Cos ........ Lichpin Paris White Cos......... Lineal Perpetual .............. Lineart Philadelphia Butter ....... Liner Royal Summer Cabbage .... Liquid St. Louis Market .......... Lizard Salamander............ Loadstar Silver Ball ............. Lobule Tennis Ball, Black Seeded .. Lodgment Tennis Ball, White Seeded.. Logbrook Tilton's White Star ....... Logline Trianon Cos or Celery...... Logman Tomhannock ............ Lordship Victoria Cabbage ......... Lottery Asparagus ............... Logan MARTYNIA Proboscidea, for Pickles.... Maccab

\section{MUSK MELONS}

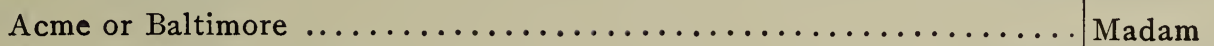

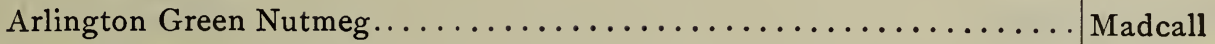

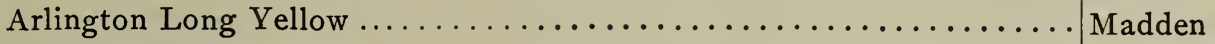

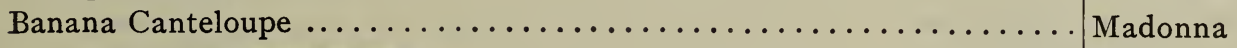

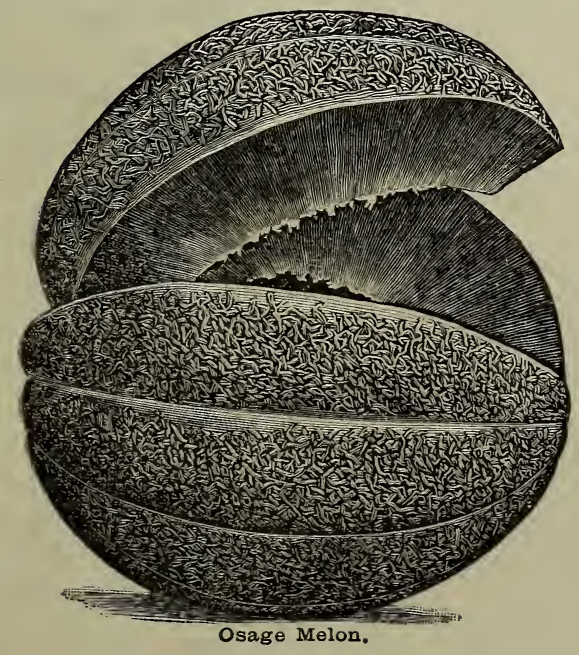

Banquet............... Magian

Bay View Canteloupe ...... Magician

Bird Canteloupe.......... Magnate

California Citron......... Magnetism

California Nectar ......... Magnific

Casaba ............................ Magnifier

Champion Market........ Maihem

Chicago Market Nutmeg.... Main

Columbus............................. Majesty

Cosmopolitan ........... Major

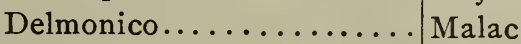

Early Christiana ......... Malapert

Early Hackensack ........ Malcon

Early Jenny Lind ......... Malefactor

Early Nutmeg .......... Maligner

Early Prolific Nutmeg ...... Malson 


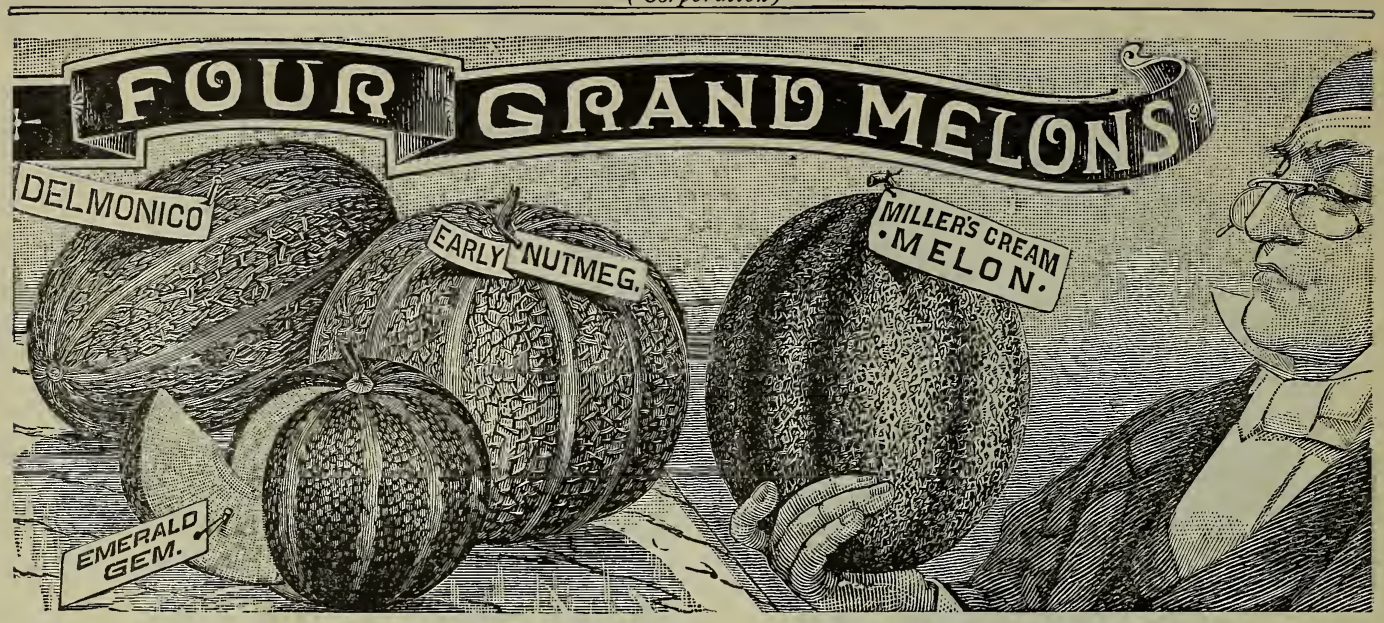

\begin{tabular}{|c|c|c|}
\hline ARTICLE & TEL. CODE & PER LB。 \\
\hline 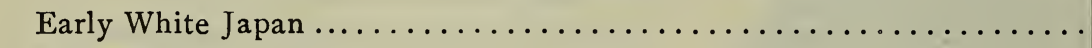 & Mall & .35 \\
\hline 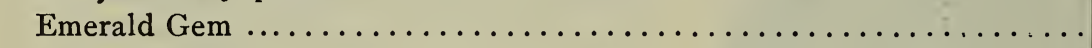 & Mallard & .40 \\
\hline Extra Eraly Green Citron $\ldots \ldots \ldots \ldots \ldots \ldots \ldots \ldots \ldots$ & Maltman & .30 \\
\hline Extra Early Hackensack... & Malster & .40 \\
\hline Golden or Netted Gem $\ldots \ldots \ldots \ldots \ldots \ldots \ldots$ & Manes & .50 \\
\hline Green Citron $\ldots \ldots \ldots \ldots \ldots \ldots \ldots \ldots \ldots$ & Mas & .30 \\
\hline 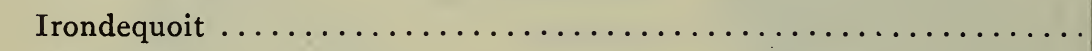 & Maritime & .35 \\
\hline Melon Peach & Mas & .60 \\
\hline 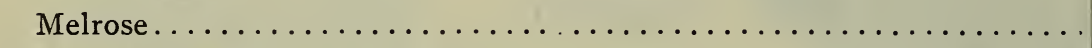 & Mas & .45 \\
\hline 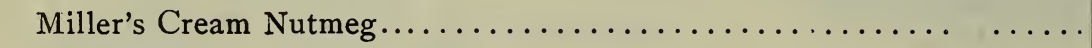 & $\mathrm{Ma}$ & .40 \\
\hline 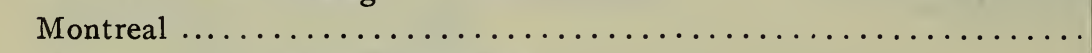 & don & .40 \\
\hline Green Nutmeg .............. & & .35 \\
\hline New Orleans Market & Mas & .40 \\
\hline 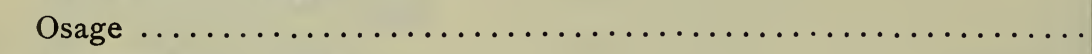 & pole & .35 \\
\hline 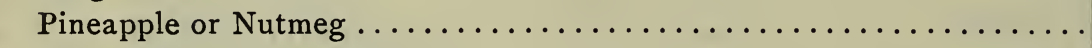 & $\mathrm{M}$ & .40 \\
\hline$\ldots \ldots \ldots \ldots \ldots \ldots \ldots \ldots$ & on & .35 \\
\hline nteloupe $\ldots \ldots \ldots \ldots \ldots \ldots \ldots \ldots \ldots \ldots \ldots$ & $\mathrm{Me}$ & .35 \\
\hline 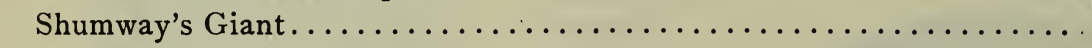 & cine & .35 \\
\hline Skil & $\mathrm{Me}$ & .35 \\
\hline 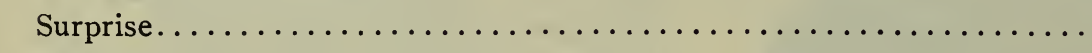 & & .35 \\
\hline $\mathrm{Ti}$ & & .40 \\
\hline Ward's Nectar.. & Memento & .35 \\
\hline \multicolumn{3}{|l|}{ WATER MELONS } \\
\hline Black Spanish & & .30 \\
\hline 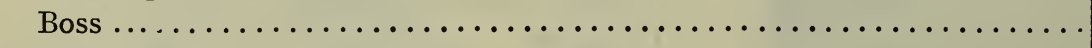 & ful & .40 \\
\hline 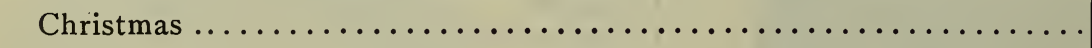 & $\mathrm{Me}$ & .35 \\
\hline 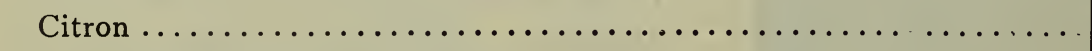 & Merm & .35 \\
\hline Citron Red Seeded.... & Messenger & .35 \\
\hline Cole's Early......... & Messmate & .35 \\
\hline Cuban Queen.......... & Meter & .35 \\
\hline Dark Icing or Ice Rind.... & Metope & .30 \\
\hline Delaware...$\ldots \ldots \ldots \ldots \ldots \ldots \ldots \ldots \ldots \ldots \ldots \ldots \ldots \ldots$ & Metric & .35 \\
\hline$\ldots \ldots \ldots \ldots \ldots \ldots \ldots \ldots \ldots \ldots$ & Metrop & .35 \\
\hline
\end{tabular}




ARTICLE

WATER MELONS - (Continued)

Florida Favorite

Fordhook Early

Midday

Georgia Rattlesnake

Midland

Midrib

Gray Monarch or Long White Icing

Militant

Henderson's Green and Gold.

Minaret

Honey 'Golden)

Minikin

Hoosier King . .

Minion

Hungarian Honey

Minnow

Ice Cream or Peerless

Mintage

Jones Jumbo

Kansas Stock Melon

Misdeed

Misnomer

Missal

Kentucky Wonder

Mission

Kolb's Gem

Moat

Mountain Sprout.

Moible

Mountain Sweet.

Mocker

Phinney's Early.

Molar

Pride of Georgia

Momentous

Rockyford

Monarchy

Ruby Gold

Monetary

Scaly Bark

Monogram

Seminole.

Monologue

Stoke's Extra Early

Mountanie

Sweetheart..

Moddiness

Vick's Extra Early

Moorland

Volga

Moppet

Duke Jones

Morris

\section{MUSTARD}

Black or Brown $\ldots \ldots \ldots \ldots \ldots \ldots \ldots \ldots \ldots \ldots \ldots \ldots \ldots \ldots \ldots \ldots \ldots \ldots$ Morbid

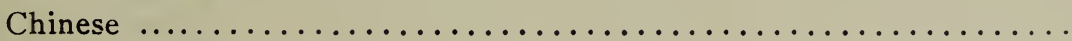

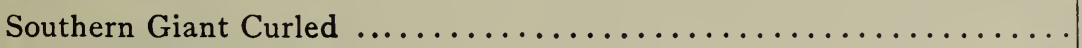

White London.

Morion

.75

.60

\section{MUSHROOM SPAWN}

Best English Milltrack........................... Mumbler

French

Mural

\section{NASTURTIUM OR INDIAN CRESS}

Dwarf Mixed

Tall Crimson

Nadir

Tall Mixed

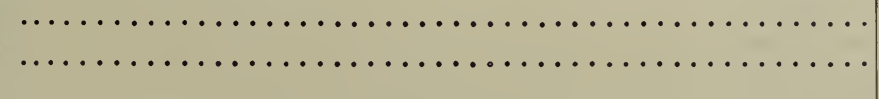

\section{OKRA}

Dwarf Prolific

Dwarf White 


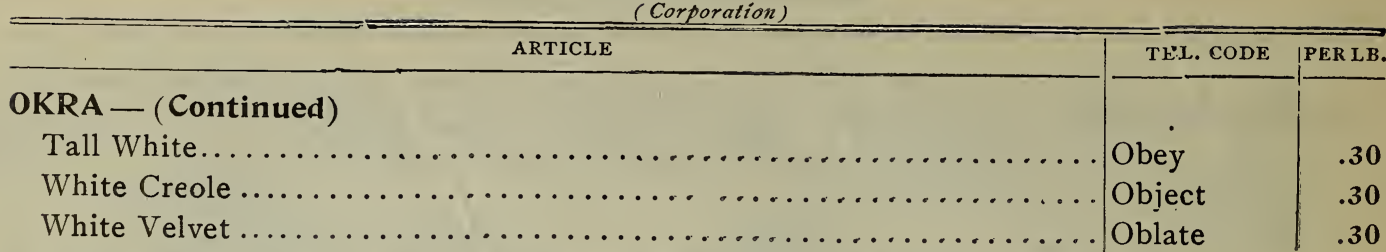

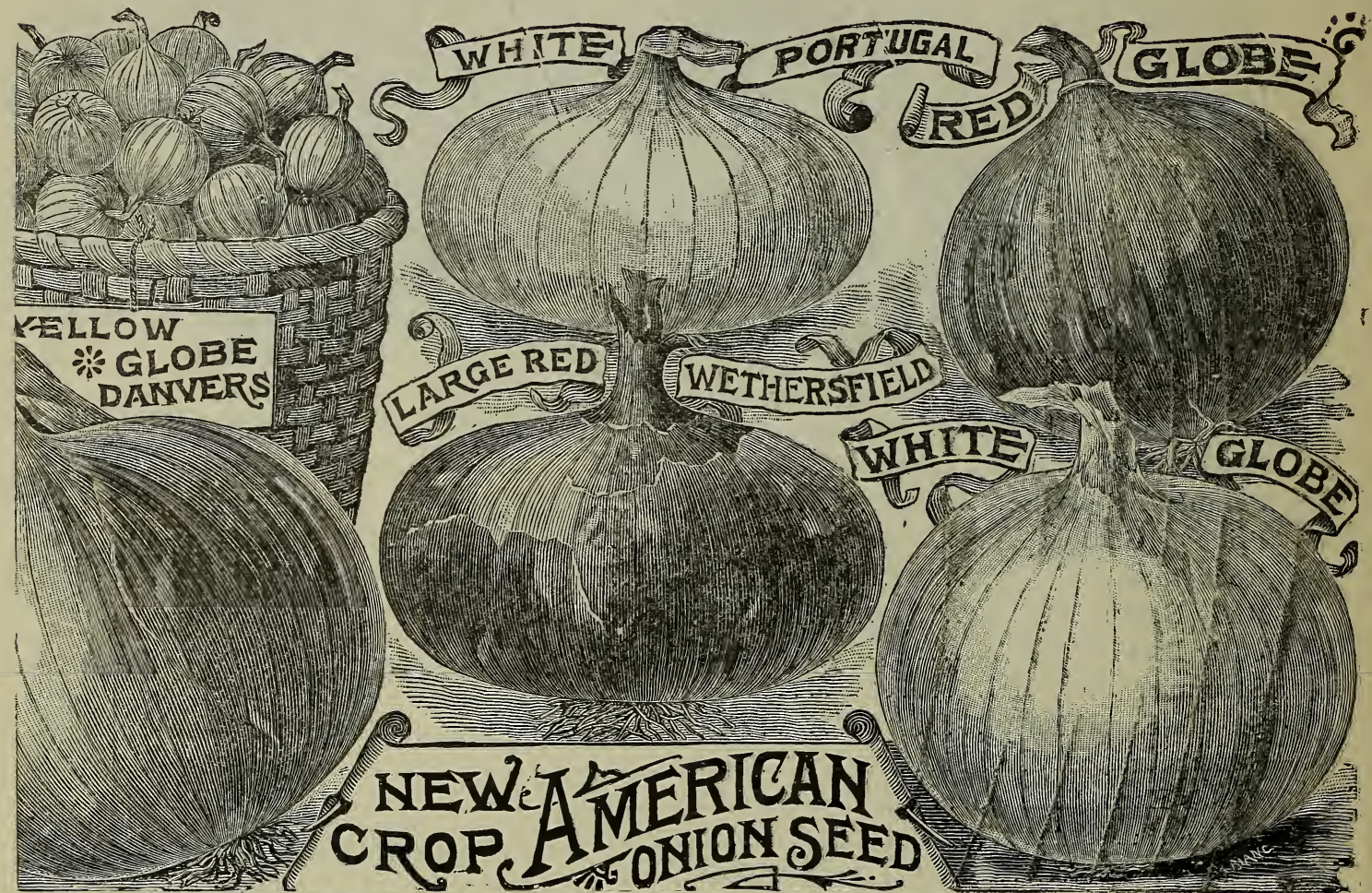

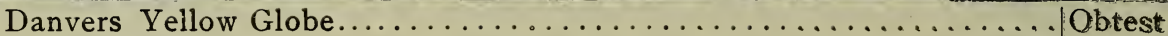

Early Red Globe ............................... Obviate

Early Round White Hard Dutch .................... Occult

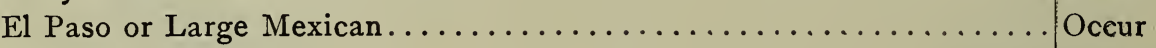

Extra Early Pearl.............................. Octave

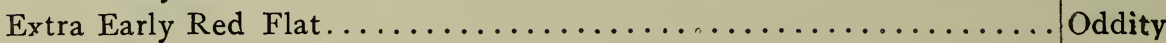

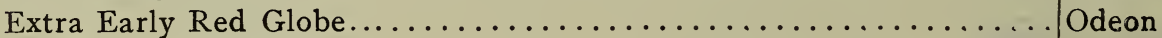

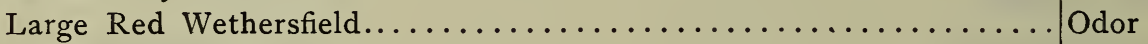

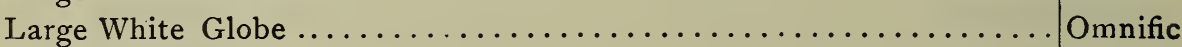

Large Strasburg or Yellow Dutch ...................... Onset

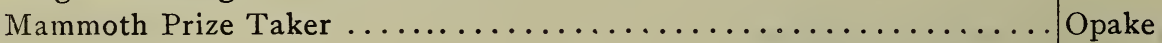

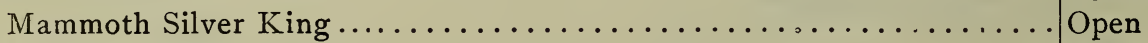

Michigan Yellow Globe............................ Opera

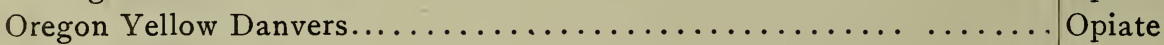

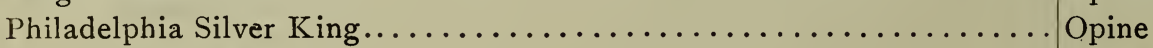

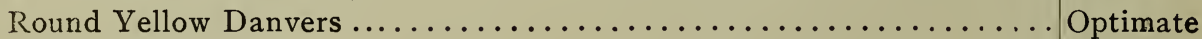

Southport Red Globe ........................... Orbit

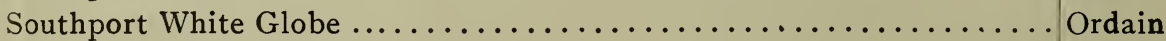

Southport Yellow Globe.$\ldots \ldots \ldots \ldots \ldots \ldots \ldots \ldots \ldots \ldots \ldots$ Ordeal

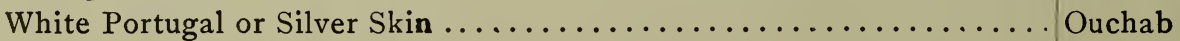

Yellow Flat Danvers............................ Outally 


\section{ONION_FOREIGN VARIETIES}

Bermuda Red

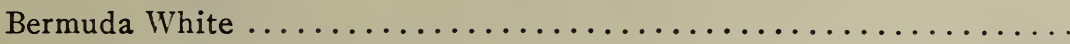

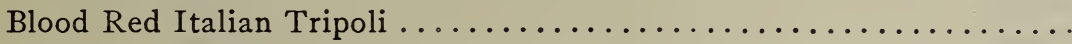

Brown St. Laurent.

Outoking $\quad 2.10$

1.85

Outoller

1.00

Outomb

1.25

Earliest White Queen

Outopping

Outoss

1.75

Early Neapolitan Marzajola

Outway

Early White Flat Italian Tripoli

Orthofic

Giant Brown Rocca

Orthofoc

Giant Rocca (Red).

Oriel

Giant White Garganus

Orison

Giant Yellow Rocca.

Orolong

Giant Vesuvius.

Orphact

Large Italian Red Tripoli . .

Orphalm

Mammoth Pompeii

Orphebe

Mammoth Red Garganus

Orphegot

Mammoth Red Victoria

Orphekle

Paris Pickling

Orphogg

Teneriffe or Bermuda

Ovade

White Bunch

Ovams

\section{PARSLEY}

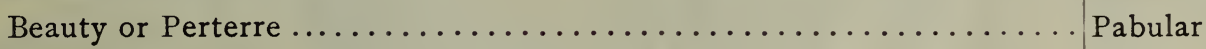

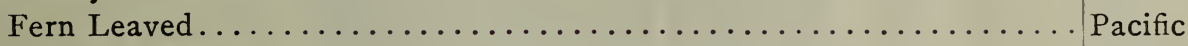

Champion Moss Curled....................................

Double Curled.

Packstaff

Emerald or Dwarf Extra Curled

Pactitions

Fine Triple Curled

Painful

Hamburg or Turnip Rooted

Palace

Plain Leaved or Single

Paleness

\section{PARSNIP}

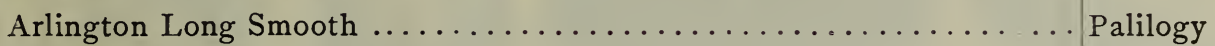

Early Short Round French

Palisade

Hollow Crown or Guernsey

Pallid

Large White or Hollow Crown

Palsical

Maltese

Palter

Magnum Bonum

Pamard

Student

Pamphlet

1.00

1.30

1.85

1.00

1.00

1.50

1.00

1.25

1.00

1.25

1.00

1.50

1.35

1.85

1.25

\section{PEPPER}

Black Nubian

Perpetuate

Cardinal

Perquisite

Celestial

Personage

Coral Gem Bouquet.

Perspire

Cranberry

Persuasion

Giant Emperor 


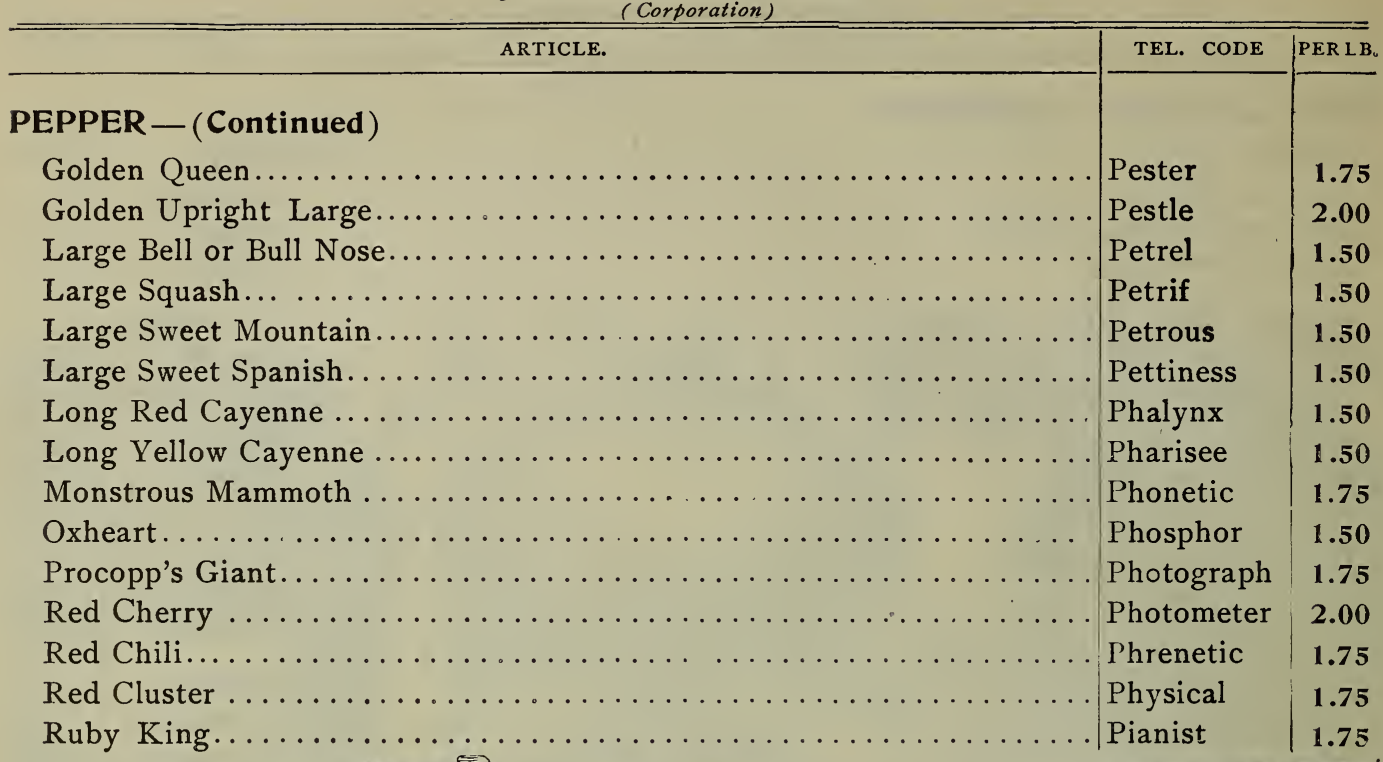

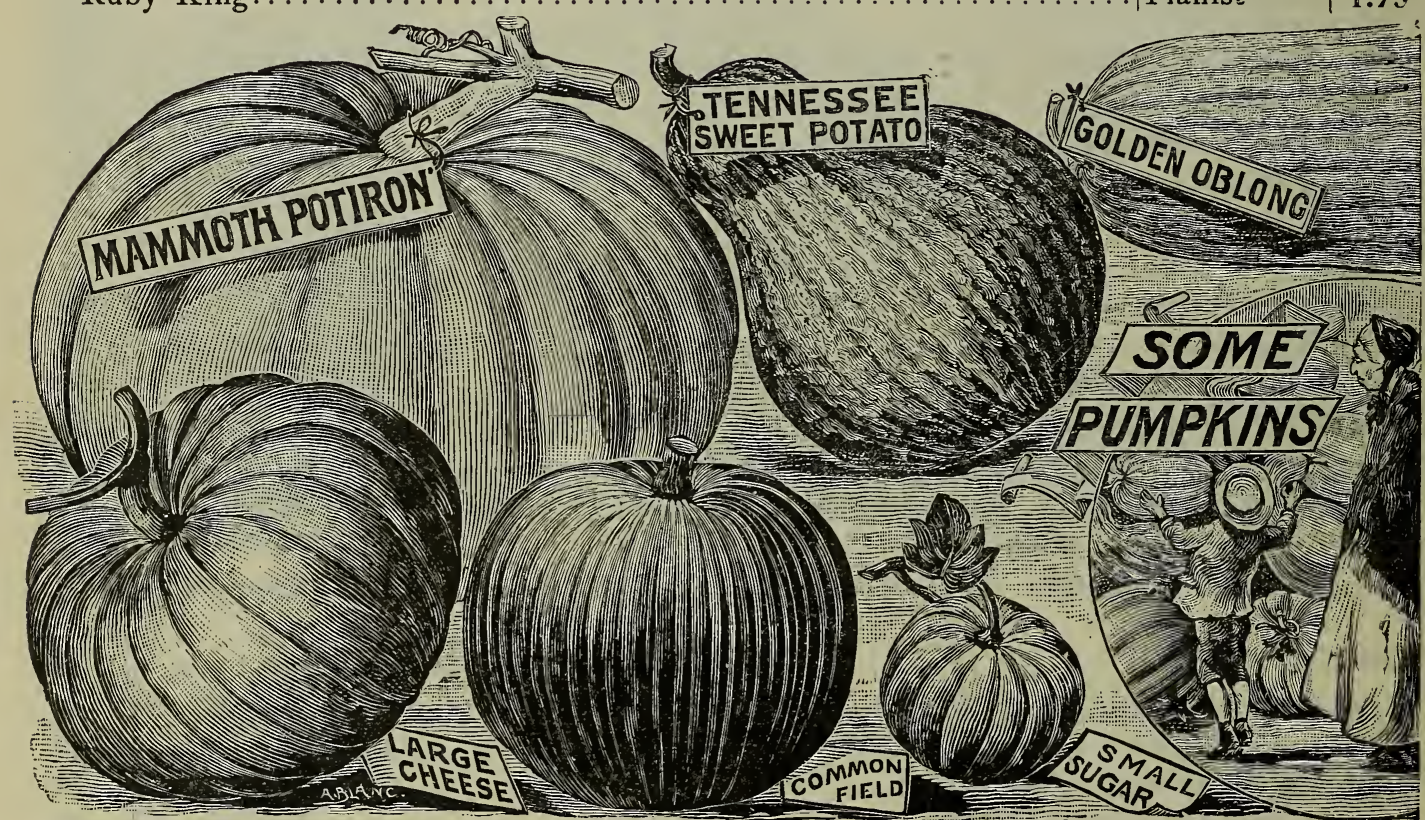

\section{PUMPKIN}

Calhou

Polygram

.40

Connecticut Field.

Polymp

Dunkard Winter.

Etampes Mammoth Red

Polytech

.14

Golden Oblong

Polythist

Improved Cashaw.

Pomifer

Jonathan

Pompac

Kentucky Field .

Pompol

King of the Mammoths

Pond

Pondag

Large Cheese...

Pondal

Mammoth or Jumbo

Pontoon

Mammoth Potiron

Popery 


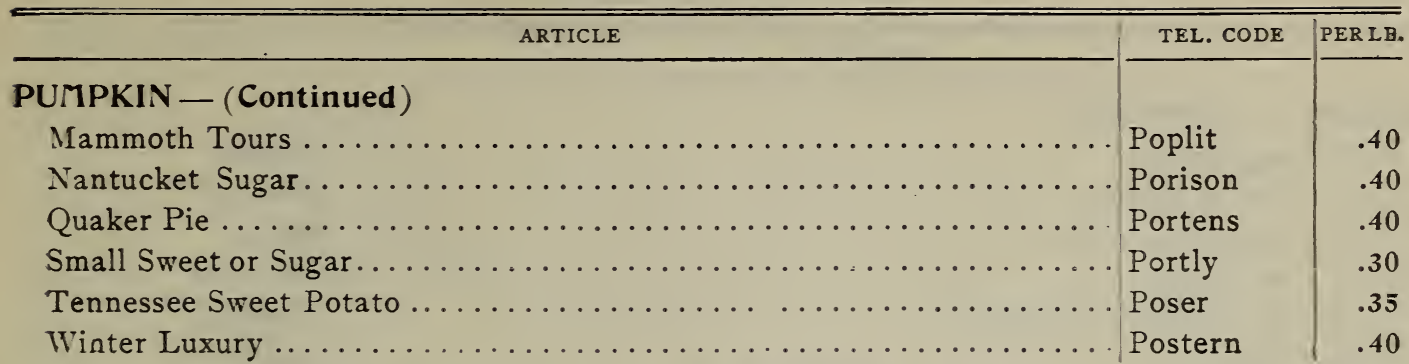

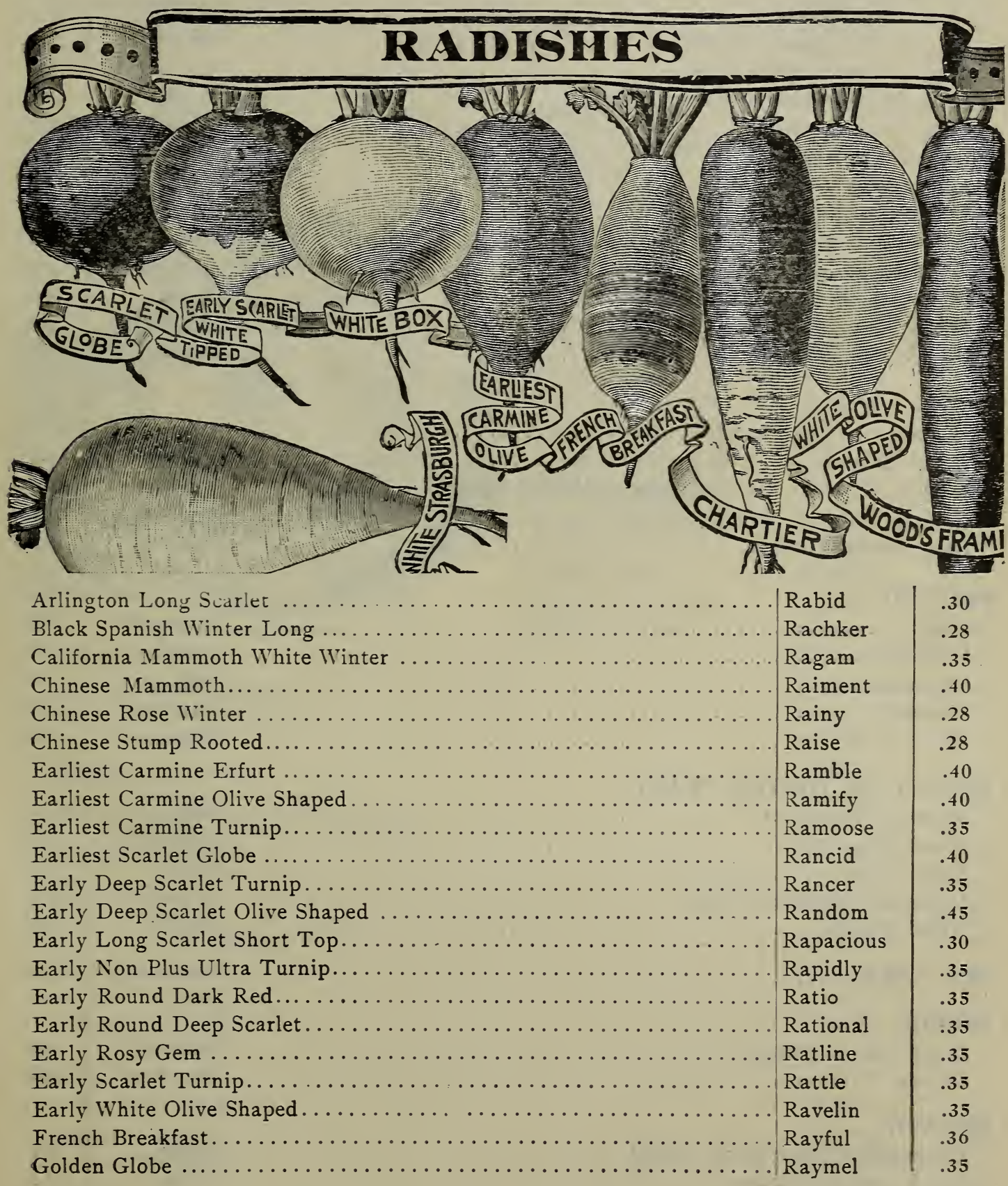




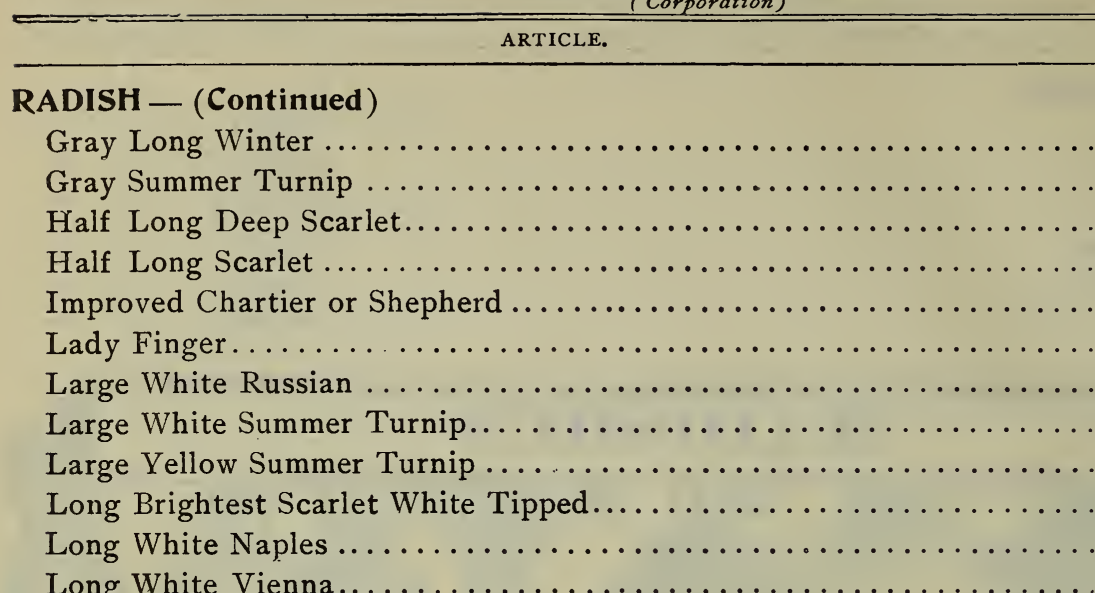

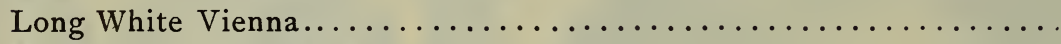

Philadelphia White Box

Raysug

Razure

Reach

Reactive

Reading

Reaffirm

Reafford

Reagable

Realism

Really

Reanal

Reapting

Receptive

Twenty Days Forcing

Refine

White Giant Stutgart

Reform

White Strasburg.

Refuple

White Tipped Scarlet Turnip....................... Regara

White Turnip.

Regent

Wood's Early Frame

Regrate

\section{RAPE}

American

Dwarf Essex

English

German

Large Seeded Garden

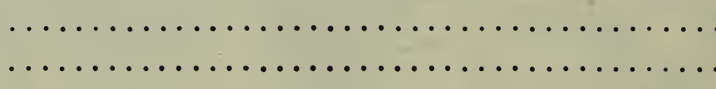




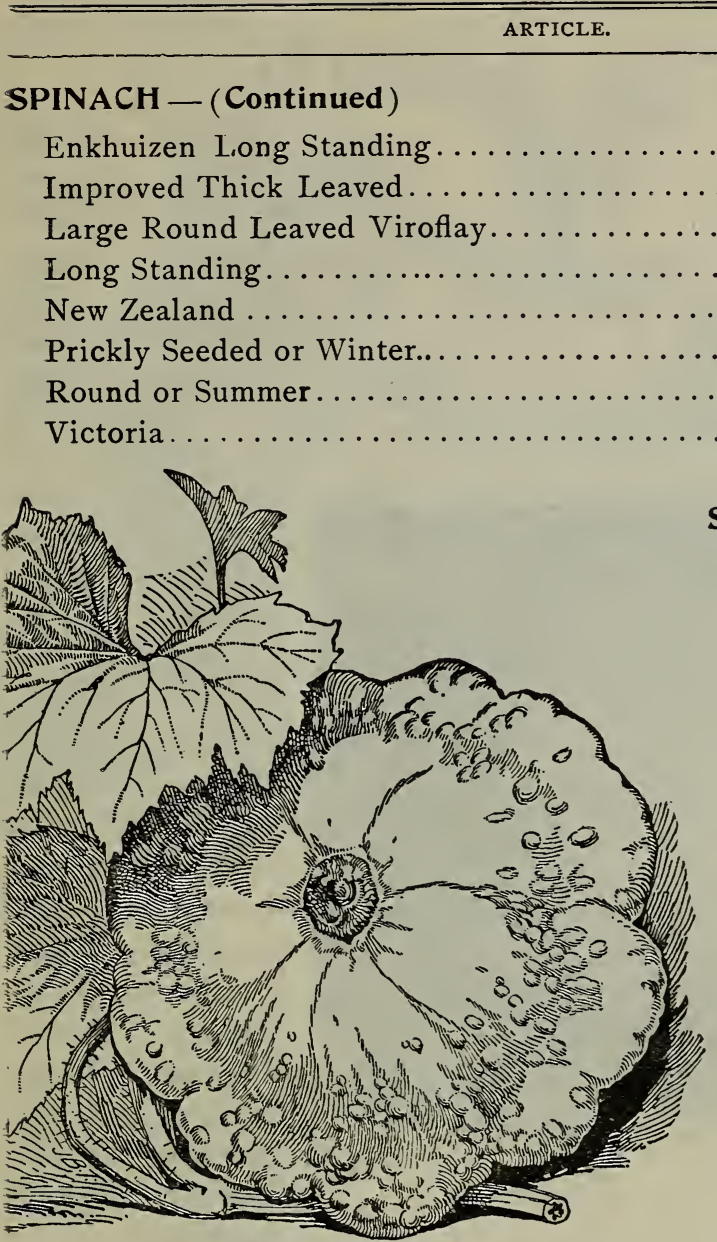

Hubbard

$$
\text { Mammoth White Bush. }
$$

SQUASH

American Turban.......

Bay State........................

Boston Marrow.........

Butman...............

Canada Crookneck......

Chicago Warty Hubbard.

Cocoanut..............

Delicata.............

Dunlap's Prolific Marrow.

Early Bush or Patty Pan.

Early Summer Crookneck

Early Fordhook.........

Early Orange Marrow....

Essex Hybrid or Turban.

Faxon ..............

Golden Bush...........

Golden Crookneck.......

Golden Custard..........

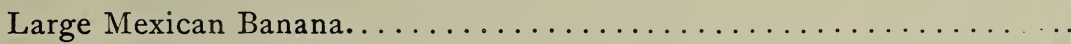

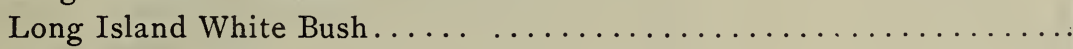

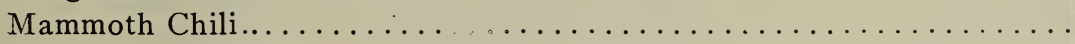

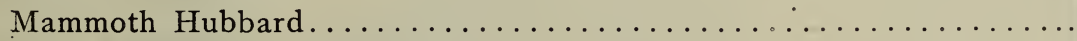

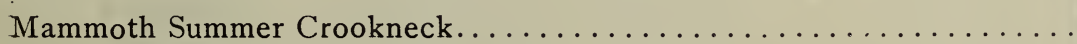

Mammoth White Bushed Scalloped.

Saltation

Samiel

Sanctity

Sandal

Sanguine

Sapphic

Satanic

Satrap

.14

.13

.13

.14

.50

.14

.13

.13

Savack

Savant

Saver

Sapit

Sawyer

Scaffold

Scalable

Scalens

Scamble

Scandal

Scant

Scape

Scarcity

Schist

Schooner

Scoffer

Scallop

Sconce

Scoundrel

.40

.40

.35

.35

.35

.40

.45

.40

.40

.35

.35

.40

.35

.40

.50

.35

.35

.40

Scripture

Scruple

Sculler

Sculptor

Scupper

Scutcheon

Scutiform

Seaborn

Marblehead

Seagage

Perfect Gem

Sealegs

Pike's Peak or Sib
Vegetable Marrow

Sectary

White Pineapple.

Sediment

Winter Crookneck

Sedition

.35

.40

.35

.70

.40

.40

.35

.40

.35

.40

.35

.40

.40

.35

\section{TOBACCO}

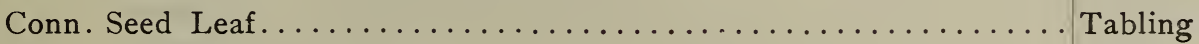

General Grant. . . . . . . . . . . . . . . . . . . . . . . . . Taboret

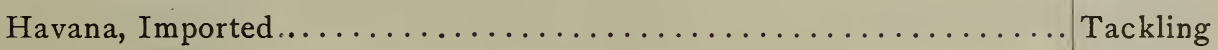

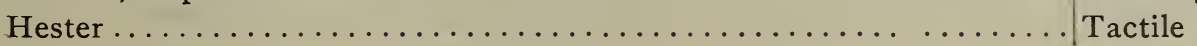




\begin{tabular}{|c|c|c|}
\hline ARTICLE & TEL. CODE & PER I.R \\
\hline \multicolumn{3}{|l|}{ TOBACCO - (Continued) } \\
\hline Lancaster Co., Broadleaf & Tailoress & 1.50 \\
\hline Missouri Broadleaf. . & Talcose & 1.50 \\
\hline Sweet Oronoko...... & Talus & 1.50 \\
\hline Tennessee Red.... & Tambour & 1.50 \\
\hline White Burleigh..... & Tandem & 2.50 \\
\hline Wisconsin Hybrid... & Tangible & 1.50 \\
\hline Zimmer's Spanish... & Tantrum & 1.50 \\
\hline \multicolumn{3}{|l|}{ TOMATO. } \\
\hline Acme...$\ldots \ldots$ & Tapan & 1.50 \\
\hline Atlantic Prize.... NEW & Tapster & 1.50 \\
\hline Beauty.......... & Target & 1.50 \\
\hline Boston Market .... & Tarker & 1.50 \\
\hline Buckeye State.... & Tarnish & 1.75 \\
\hline Canada Victor..... & Tarry & $1 \cdot 50$ \\
\hline Cardinal ......... & Tartan & 1.40 \\
\hline Conqueror......... & Taster & 1.40 \\
\hline Crimson Cushion... & Tattle & 1.50 \\
\hline Dwarf Champion... & Taurus & 1.50 \\
\hline Early Essex Hybrid & Tawny & 1.50 \\
\hline Early Mayflower.... & Taxidermy & 1.25 \\
\hline Early Michigan..... & Teacher & 1.25 \\
\hline Early Optimus...... & Teadly & 1.40 \\
\hline Early Ruby...... & Tearless & 1.50 \\
\hline Favorite ........ & Teeter & 1.40 \\
\hline General Grant.... & Telescope & 1.25 \\
\hline Golden Husk........ & Temper & $1 \cdot 75$ \\
\hline Ground Cherry or Husk. & Temptation & 1.75 \\
\hline Hathaway's Excelsior... & Tempter & 1.40 \\
\hline Ignotum . . . . . . . . & Tender & 1.50 \\
\hline Improved Trophy ...... & Tenent & 1.50 \\
\hline Large Smooth Red.. & Tentative & 1.25 \\
\hline Long Keeper........ & Tepor & 1.60 \\
\hline Lorillard........... & Temporal & 1.50 \\
\hline Mansfield Tree......... & Teraphin & 2.25 \\
\hline Matchless.......... & Termagant & 1.60 \\
\hline Mikado or Turner's Hybrid & Terminus & 1.65 \\
\hline Paragon & Terror & 1.50 \\
\hline Peach $. . . . . . . . \ldots .$. & Tertial & 1.50 \\
\hline Pear-shaped Red... & Testable & 1.75 \\
\hline Pear-shaped Yellow. & Testimony & 2.00 \\
\hline Ponderosa ......... & Testudo & 2.75 \\
\hline Red Cherry ........ & Textual & 1.75 . \\
\hline Red Currant....... & Thane & 2.00 \\
\hline Royal Red........ & Theatric & 1.75 \\
\hline Stone $\ldots \ldots \ldots \ldots \ldots$. & Thesis & 1.50 \\
\hline Strawberry or Ground Cherry & Theurgy & 1.75 \\
\hline Yellow Cherry......... & Thong & 2.25 \\
\hline
\end{tabular}



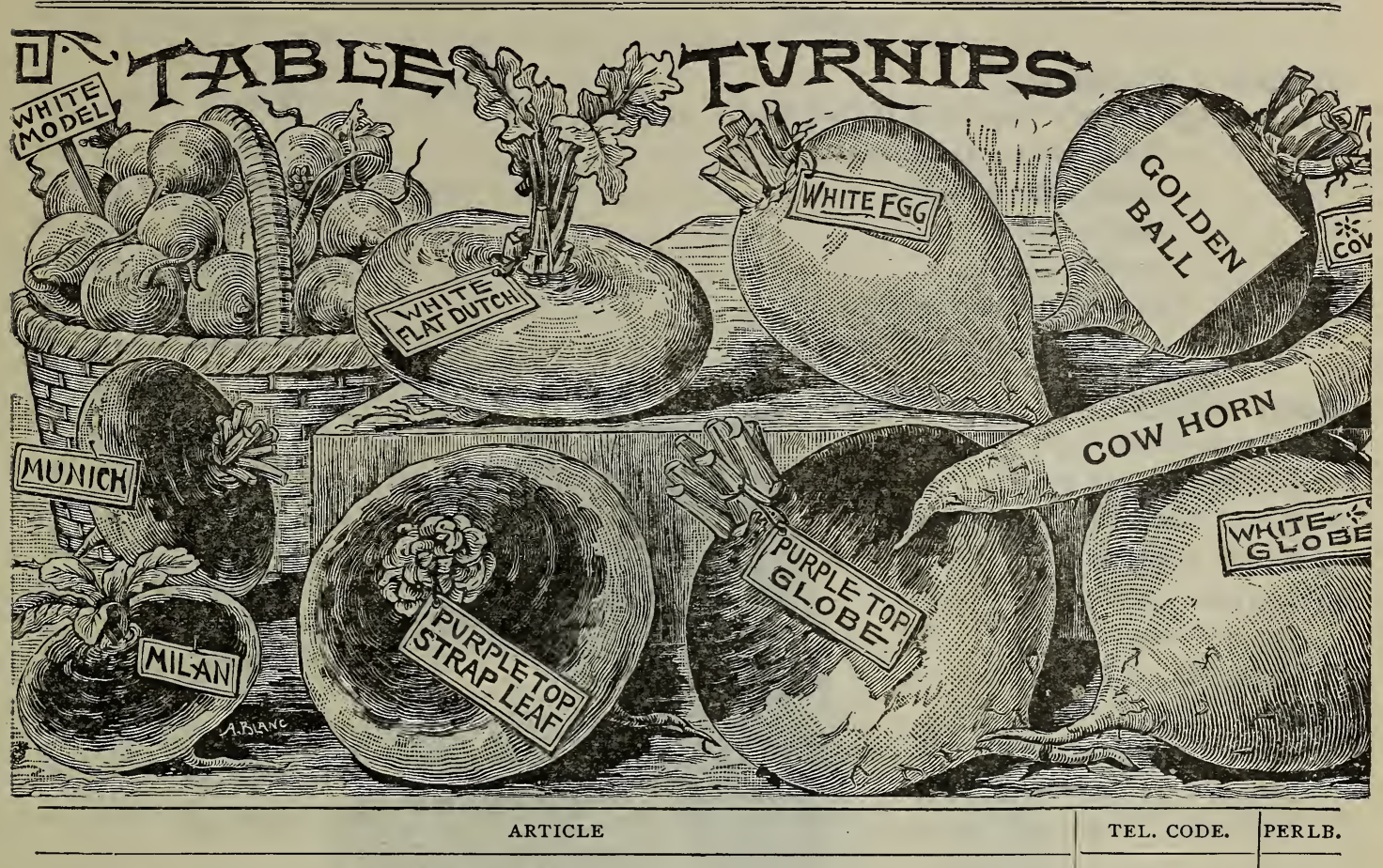

Amber Globe............................... Thrice

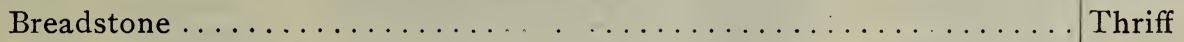

Early Purple Top Milan . . . . . . . . . . . . . . . . . . . . . Thugen

Early Purple Top Munich . . . . . . . . . . . . . . . . . . Thuging

Early Red or Purple Top Strap Leaf................... Thugord

Early Red Top White Globe ...................... Thugul

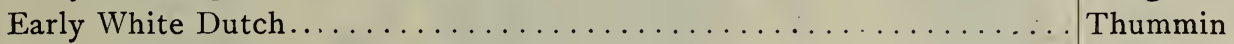

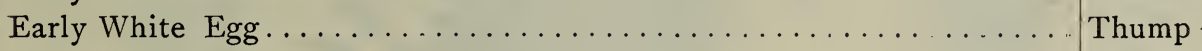

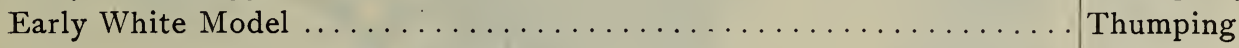

Early White Six Weeks or Snowball ................... Thumpork

Early White Strap Leaved....................... Thwack

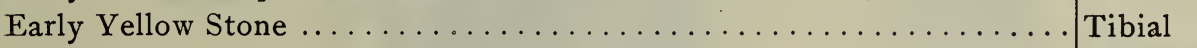

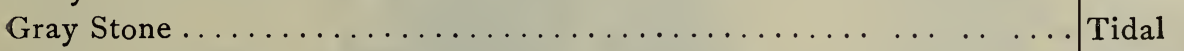

Green Top Yellow Aberdeen $\ldots \ldots \ldots \ldots \ldots \ldots \ldots \ldots \ldots \ldots \ldots \ldots$ Tidily

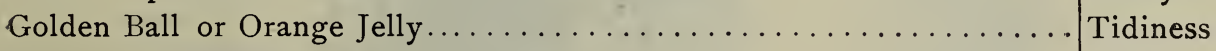

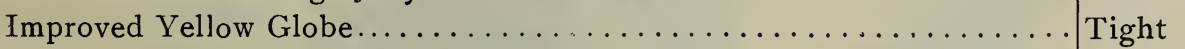

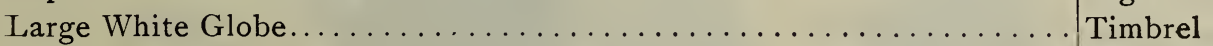

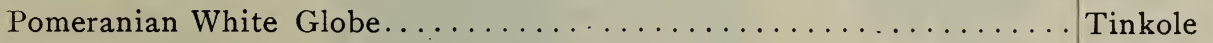

Purple Top Yellow Aberdeen........................ Tiresome

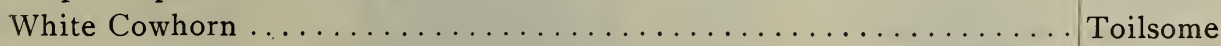

White Flat Norfolk ............................ Tolerant

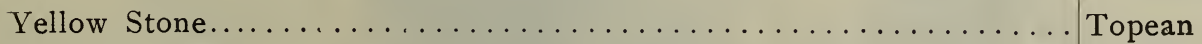

Ruta-Baga Budlong ............................. Topknot

" Carter's Hardy Swede (Imperial)....

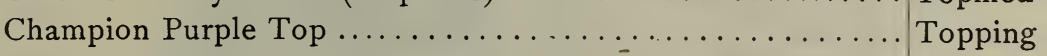

" Hall's Westbury....................... Torel

" Hurst's Monarch........................ Tornado

“ Improved American Purple Top. Tornado
Torpor 


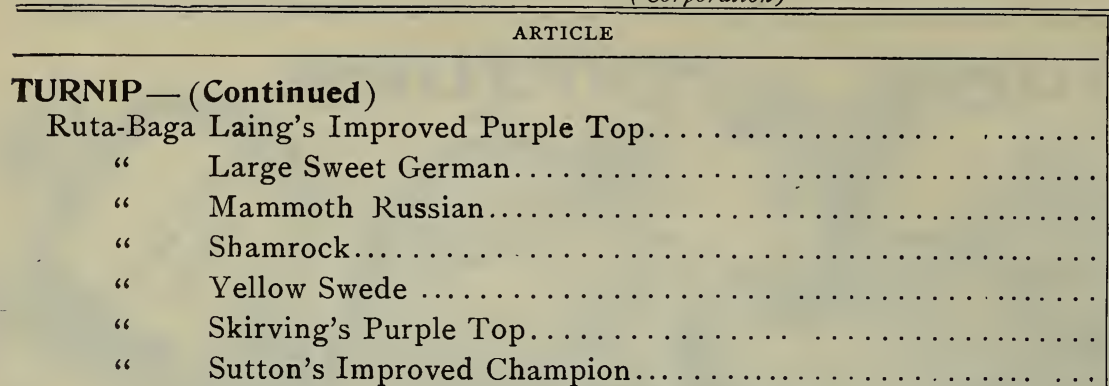

\section{BIRD SEEDS}

Canary, Sicily Bulk

Canary, Smyrna Bulk

Hemp, American or Domestic.....

Hemp, Russian..............

\begin{tabular}{l} 
TEL. C \\
\hline Torrid \\
Tortive
\end{tabular}

Touching

Tournial

.22

.26

Tousel

.26

Towning

.26

.26

Lettuce.

Tractile

Pigeon Peas
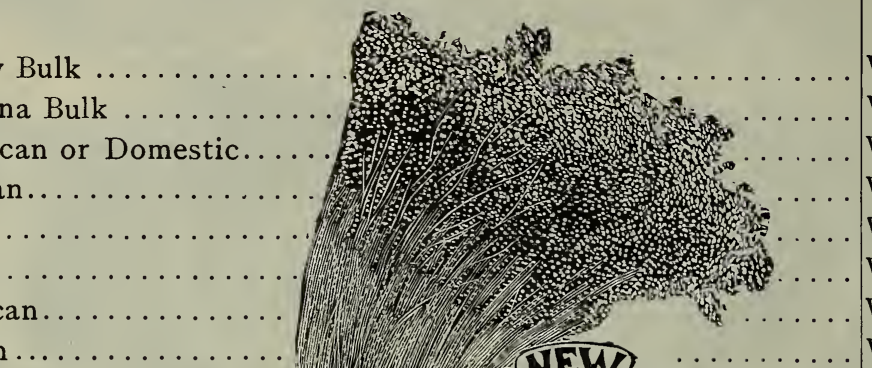

Rape, English

Rape, German .

Sunflower, Common Black.

Sunflower, Mammoth Russian

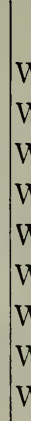

Whecker

Wheek

Whide

Whief

Whieftain

$.3 \frac{1}{4}$

.03

.04

$.3 \frac{1}{4}$

.15

.02

$.3 \frac{1}{2}$

Whirp

Whival

Whock

$.4 \frac{1}{2}$

$.3 \frac{1}{4}$

.04

$.3 \frac{1}{2}$

\section{MISCELLANEOUS SEEDS}

Broom Corn, California Golden

Broom Corn, Dwarf Evergreen ....
Broom Corn, Extra Early Japanese

Broom Corn, Extra Early Japanese
Broom Corn, Improved Evergreen

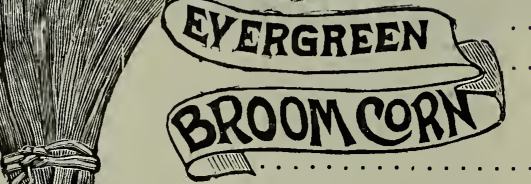

Whosen

Whough

Whowder

.08

.06

.08

.06

Broom Corn, Tall Evergreen ......

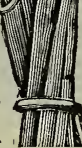

Whrism

Whristen

Whristian

Whromat

.08

Castor Beans

Corn, Sweet for Parching..

Flax, Russia
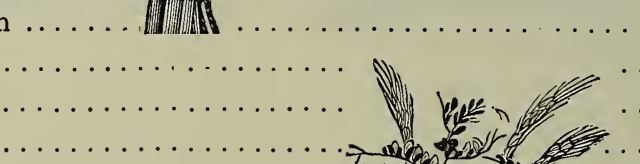

Kidney Vetch or Sand Clover.

Lentils

\section{FORAGE PLANTS}

Dourrha Brown.

Dourrha Guinea Corn

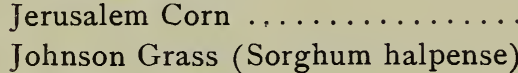

Kaffir Corn, Red

Kaffir Corn, White

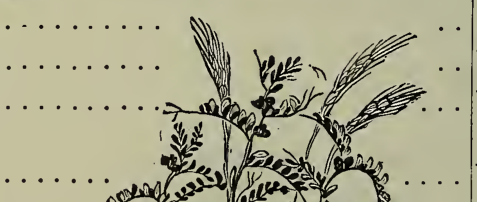

Whug

Whubby

Whunk

Wilia

Wilic

Lathyrus Silvestris (Flat Pea)

Millo Maize

Millo Maize White Branching

Millo Maize Yellow...

Sacaline (Polygonum sachalinense).

Sandvetch (Vicia villosa)

Spurry (Spergula arnensis maxima)......

Teosinte (Reana luxurians) ............

Vetches, Spring

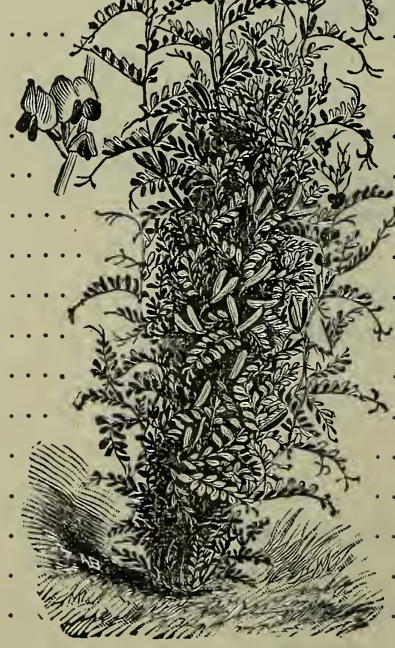

Spring Vetches.

Wivil

Wivisen

Woast

Wobble

Wobweb

Wochal

Wockhat

Woddle

Wodger

Wodic

Wodlin

Woffer

Woffle

Wogent

Wognate

.08

.08

.08

.12

.08

.08

1.00

.08

.08

.08

4.00

.10

.12

.80

$.5 \frac{1}{4}$ 


ARTICLE

FORAGE PLANTS-(Continued)

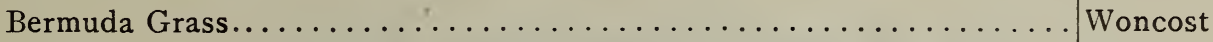

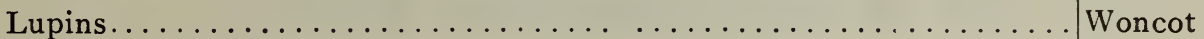

Soya or Coffee Bean

Woncorn

Early Amber Cane.

Wondern

Early Orange Cane

Wondit

.45

.10

.06

.08

.08

\section{BARLEY}

Black

Wohere

Chevalier

Woif

Mansury

Wollab

Spring

Wollate

Two Rowed

Wollege

\section{BUCKWHEAT}

American Silver Hull 2 की

Common

Japanese

\section{e.}

\section{OATS}

Early Archangel .

American Banner.

American White

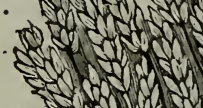

Lincoln

Racehorse

Welcome

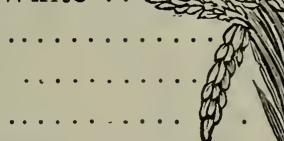

White Bedford

White Russian

\section{RYE \\ Spring \\ Winter \\ WHEAT_-SPRING}

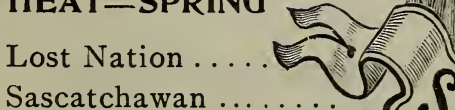

Sascatchawan .......

Velvet Chaff Blue Stem.

White Russian

\section{WHEAT_-WINTER}

Clawson ........ N

Clawson, White ... ते

Fultz...........

Martin Amber
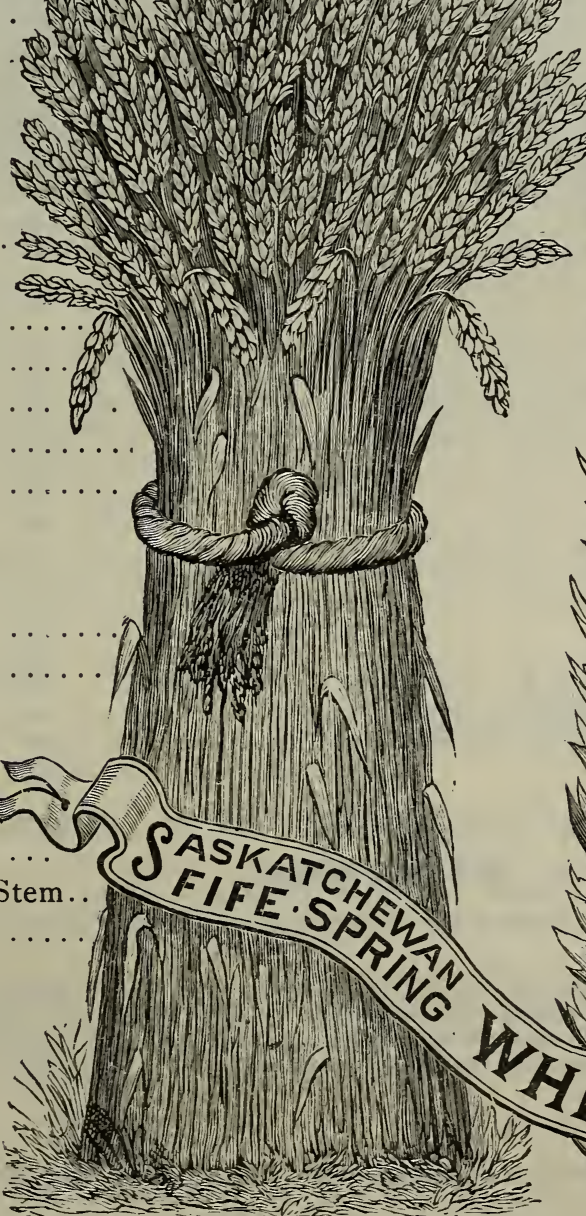

Mediterranean
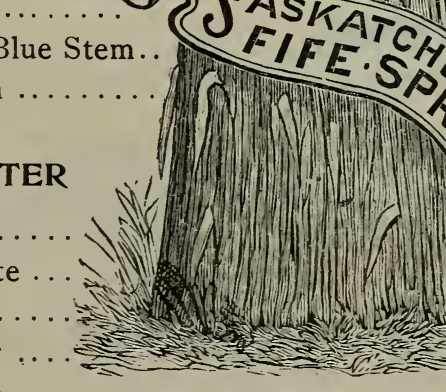


\section{JOSEPH BRECK \& SONS,}

(Corporation.)

47 to 54 North Market Street, Boston.

\section{GRASS ST
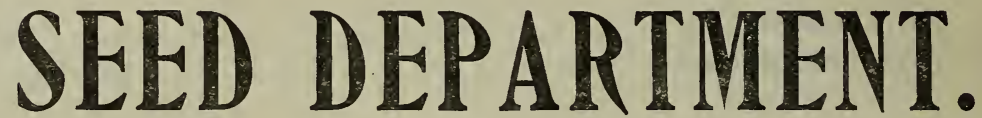

Orders wired or mailed on receipt of this Price-List promptly executed at prices named.

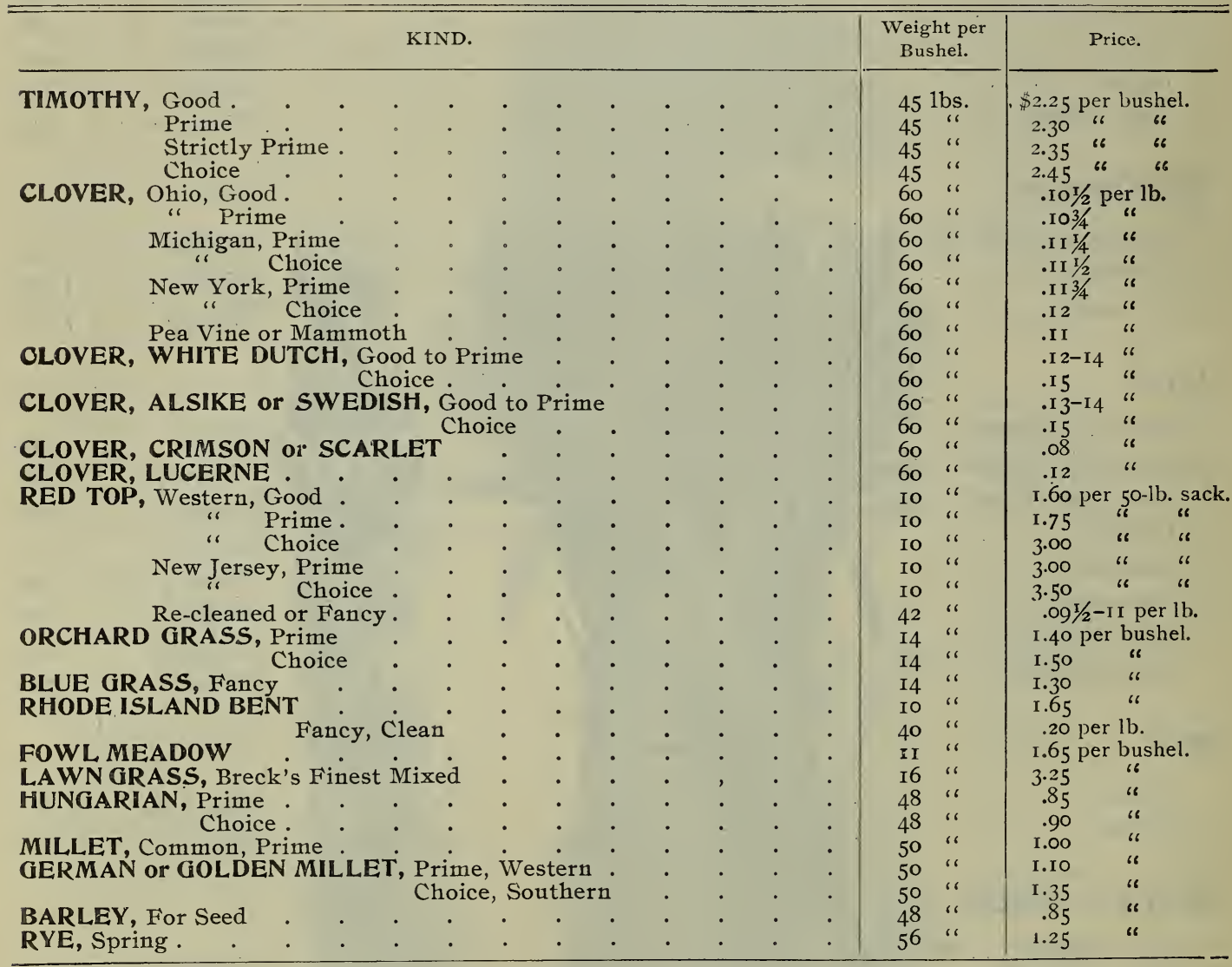

\section{REMARKS.}

TIMOTHY. Brisk demand expected. Stocks are mostly out of growers' hands and higher prices may be looked for.

RED TOP. Stocks are of good quality and apparently small. At present, prices favor buyers.

CLOVER. The I900 crop was of fair quality, but not large. Higher prices are likely.

BLUE AND ORCHARD. These are in shorter supply than a year ago and higher prices prevail.

HUNGARIAN AND MILLETS. There is little demand for these at present. Higher prices may be looked for.

OTHER KINDS. Steady as quoted.

When in want please write or wire for quotations and we will reply promptly, making low prices. 


\section{BRECK'S LAWN GRASS SEED.}

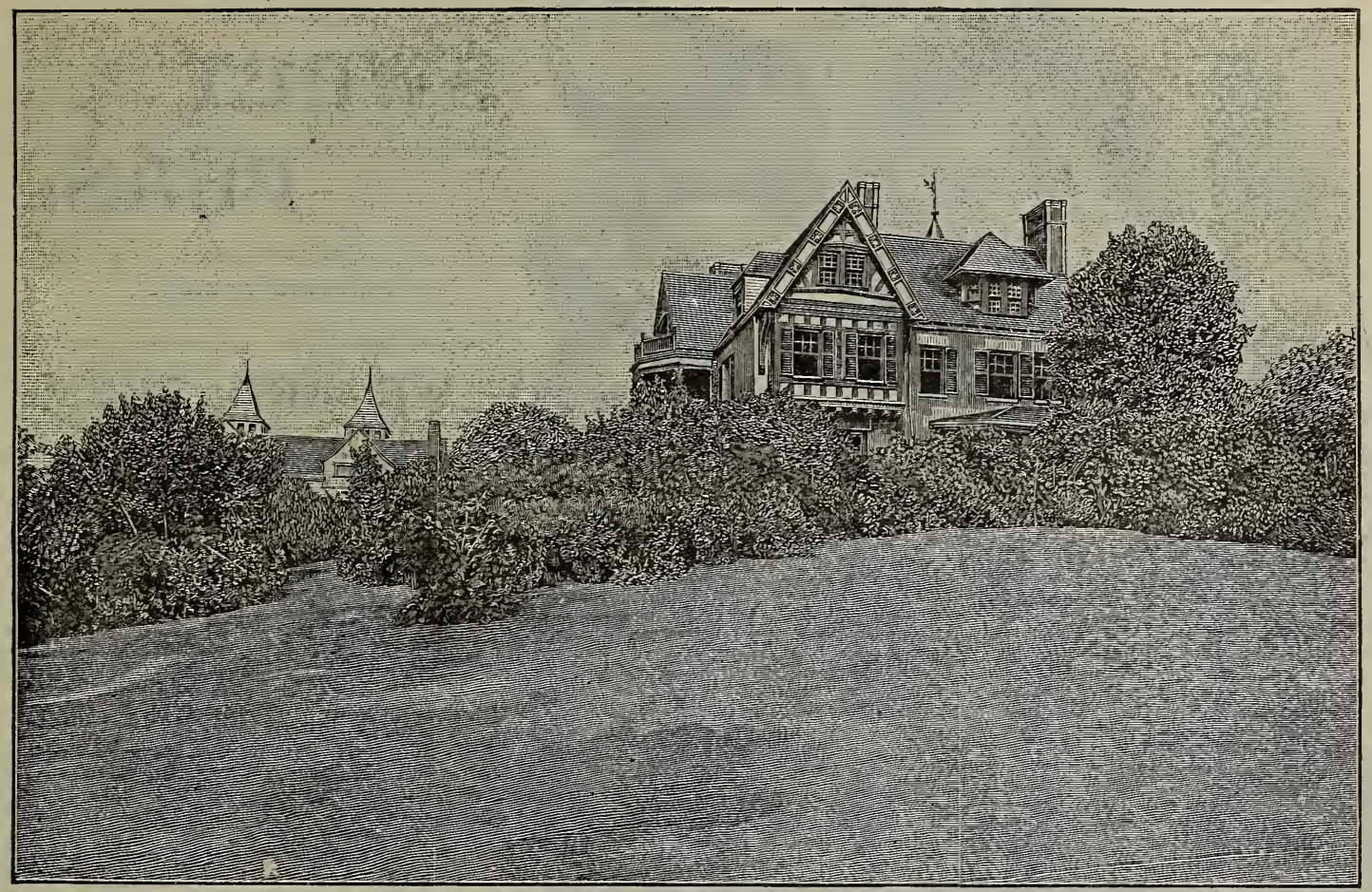

By the courtesy of Luther Adams, Esq., of Newton, Mass., we produce here a partial view of his beautiful grounds. The fine lawns on Mr. Adams' estate are the product of BRECK'S LAWN CRASS SEEDS, and are but a fair representation of thousands of lawns raised from the same UNEQUALLED MIXTURE.

We have given much thought and made many experiments in order to ascertain just what is the best mixture for permanent lawns, and the grasses which are best suited to this climate. By the proper blending of the varieties which constitute Breck's Lawn Grass, we have obtained a mixture that from early spring to late fall will give to lawns made with it that rich, deep green so often admired upon the lawns and parks of England, but so seldom seen in this country.

$$
\begin{aligned}
& \text { Per case of } 25 \text { quarts . . . . . . . . \$3.00 } \\
& \text { Per bushel, in bulk . . . . . . . . } \quad \text {. } 3.25
\end{aligned}
$$

We can supply other mixtures of so-called Lawn Grass Seed at competitive prices, but we recommend our own as being superior in every respect to all others. When desired we make special mixtures of Grasses to suit different soils and conditions.

\section{BRECK'S "SHADY SPOT" LAWN GRASS.}

This is a mixture of grasses that are naturally adapted for growing under trees and in places where the sun does not find ready entrance. If sown on such locations, in place of the ordinary Lawn Seed, bare and unsightly spots will be prevented. Per bushe1, $\$ 3.25$.

\section{RRECK'S BANK AND TERRACE GRASS MIXTURE.}

A mixture of deep-rooting grasses specially prepared for embankments and situations where it is impractical to get a successful catch of the less spreading rooted sorts. It produces a rich velvety turf throughout the season. No coarse growing undesirable varieties are used in this mixture. Per bushel, $\$ 3.25$.

\section{GOLF LINKS GRASS MIXTURE.}

Since the game of Golf became so popular in this country, we have had frequent demands for mixtures of grass seeds, suitable both for the Courses and Putting Greens, and adapted to low, damp, as well as the thinner dry soils on the elevations.

It will be readily understood that no one mixture would be suitable for the different conditions. We have, therefore, prepared three mixtures.

No. I. For Putting Greens. Sow at the rate of eighty pounds to the acre. Per 1 b., I 2 cents.

No. 2. For light and dry soils. Sow at the rate of fifty pounds to the acre. Per 1b., 12 cents.

No. 3. For low and damp grounds. Sow at the rate of fifty pounds to the acre. Per 1 b., Io cents.

We will be glad to make special mixtures for other conditions of soil, etc. 


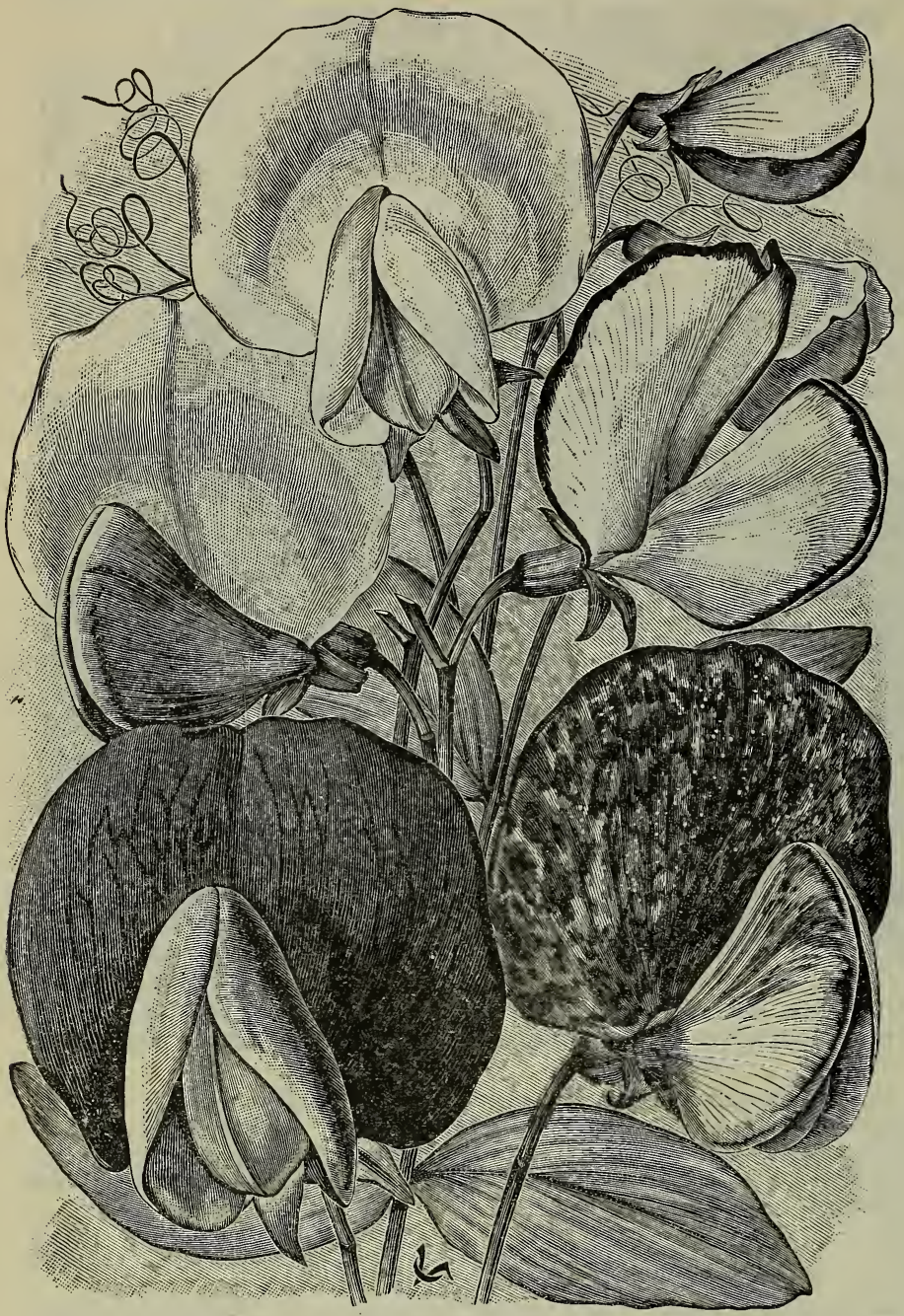

\section{SWEET \\ PEAS.}

\section{WE ARE THE}

\section{GROWERS.}

We beg to call special attention to the list of Sweet Peas offered below. All of the varieties named were grown during the past season under our personal supervision. No seed grower or merchant can offer a purer or choicer quality of Sweet Peas than those we list. They are not the product of unselected volunteer crops, as many of those offered at low prices are:

Breck's Boston Mixture. (This embraces 60 of the best varieties and is the finest mixture ever offered) ......

Choice Mixture. Contains many delicate shades .................

Eckford's Mixture. Very fine.......

Collection, 6o named sorts ..........

Collection, 25

Blanche Burpee. White .........

Emily Henderson.

Fisher's Snowflake. " $\ldots \ldots \ldots \ldots$

Mrs. Sankey.

Golden Gleam. Yellow ...........

Mrs. Eckford.

Prima Donna. Pink.............

Lovely.

Katherine Tracy. " .............

Lady Penzance. Rose ............

Ovid.
Per lb.

.50

.30

.35

.40

.30

.30

.35

.30

.30

.25

.25

.30
Her Majesty. Rose............. .25

Brilliant. Scarlet.............. 30

FirefIy. " $\quad \ldots \ldots \ldots \ldots \ldots \ldots \ldots . .30$

Harvard. Crimson ............ .30

Salopian. " $\quad \ldots \ldots \ldots \ldots \ldots \ldots . . .6 \ldots$

Mars. " $\quad \ldots \ldots \ldots \ldots \ldots \ldots . .35$

Prince Edward of York. Scarlet-crimson .30

Princess Victoria. “ $\quad .25$

Captain of the Blues. Bluish ....... .30

Lady Nina Balfour. Mauve-bluish.... .35

Celestial. "

New Countess. " "

Princess May. " "

Emily Eckford. Mauve-reddish...... .25

Captivation. Magenta ........... .25

Duke of Clarence. Claret .......... .25

Shahzada. Maroon and tints........ .30

Stanley. " " " 


\section{SWEET PEAS - (Continued)}

\section{BLENDINGS.}

Crown Jewel. Delicate creamy white, veined with violet.............

Coquette. Primrose, tinted with apricot

Venus. Salmon-buff .............

.30

$\cdot 30$

\section{STANDARD AND WINGS OF SLIGHTLY} DIFFERENT TINTS.

Lady Beaconsfield. Pink and primrose

Triumph. Orange-pink, wings white, slightly flushed $\ldots \ldots \ldots \ldots \ldots \ldots$.

Emily Lynch. Creamy rose, primrose tinted wings $\ldots \ldots \ldots \ldots \ldots \ldots \ldots$

\section{FLAKED AND CLOUDED.}

Royal Rose. Pink and blush white.... Apple Blossom. Pink and blush......

\section{RICH METALLIC.}

Countess of Powis..............

Meteor. Orange-salmon, veined with pink and purple................ Rising Sun. Orange-rose, shaded pink Etna. Crimson, marbled edges........

\section{SUFFUSED AND NARROWLY EDGED WITH LAVENDER.}

Lottie Eckford. Rose-white, blue edge $\cdot 30$

Maid of Honor. Pale blue and white shaded

Golden Gate. Edged with lavender...

\section{SUFFUSED AND NARROWLY EDGED WITH ROSE.}

Countess of Aberdeen. White, edged with rose

\section{PENCILLED EFFECT.}

Per lb.

Capt. Clark. Tricolor............ .25

Columbia. Tricolor ........... .35

CONTRASTING, STANDARD AND WINGS IN DIFFERENT TINTS.

Little Dorrit. Carmine, tinted pink, white wings $\ldots \ldots \ldots \ldots \ldots \ldots \ldots . \quad .25$

Blushing Bride. Rose and white .... .30

Earliest of All. Similar to Extra Early Blanche Ferry but ten days earlier....

Extra Early Blanche Ferry. Pink and white ........................

Duke of York, Rose, primrose wings.. .30

VARIEGATED.

Aurora. Orange-salmon flakes on white ground ...................

Mrs. Joseph Chamberlain. White, flaked with rose............. $\quad .25$

Mikado. Orange cerise, striped white.. .30

Ramona. Creamy white, splashed with pink .................... $\quad .25$

Striped Celestial. White, mauve striped .35

Gray Friar. Watered purple or white. .25

Senator. Striped chocolate or white... .25

America. Striped white and scarlet... $\quad .25$

Pink Cupid. Height, six inches ...... . .50

White Cupid. Height, six inches..... .50

Beauty Cupid. Pale rose, striped with carmine..................... $\quad .75$

Primrose Cupid. Pale primrose......

Red Ridinghood. Rosy pink and white, curiously hooded .............. .30

Bride of Niagara. Double flowered,

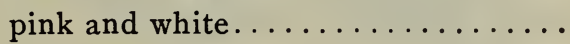

.40

\section{Boston, Mass., February, igor.}

SPECIAL.

We are row booking contract growing orders for named sorts of Sweet Peas for delivery after harvest of igor, as follows:

Pink Cupid, $30 \mathrm{cts}$. per lb.; White Cupid, $30 \mathrm{cts}$. per lb. Other varieties above listed, except the new Cupids, 17 cts. per lb., on orders of not less than ro lbs. of any variety.

Terms, net cash, 6o days from date of invoice.

It is only on contract orders that we can afford to name such low prices. Dealers who buy in round lots will surely save money by placing orders immediately. 


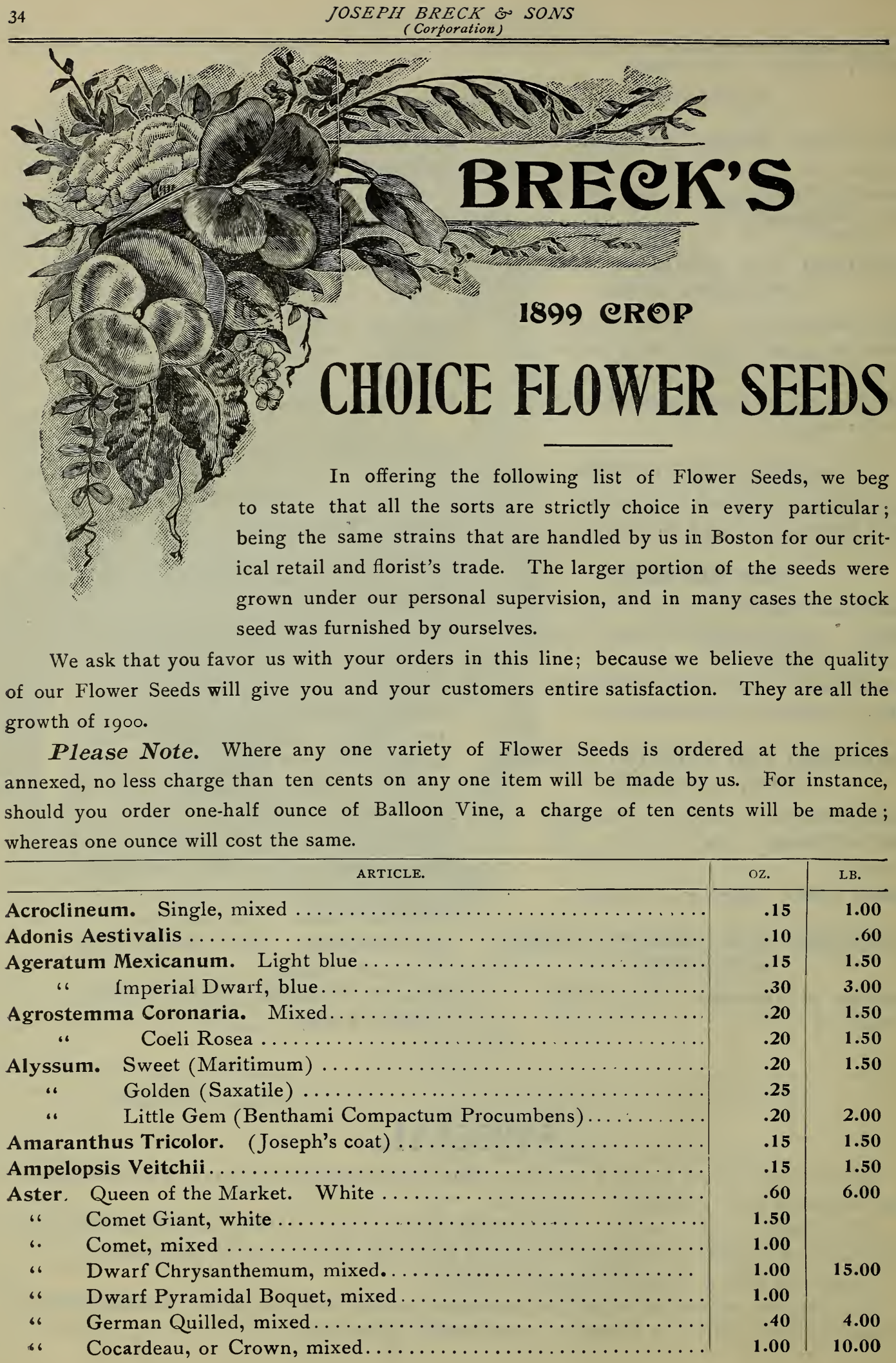




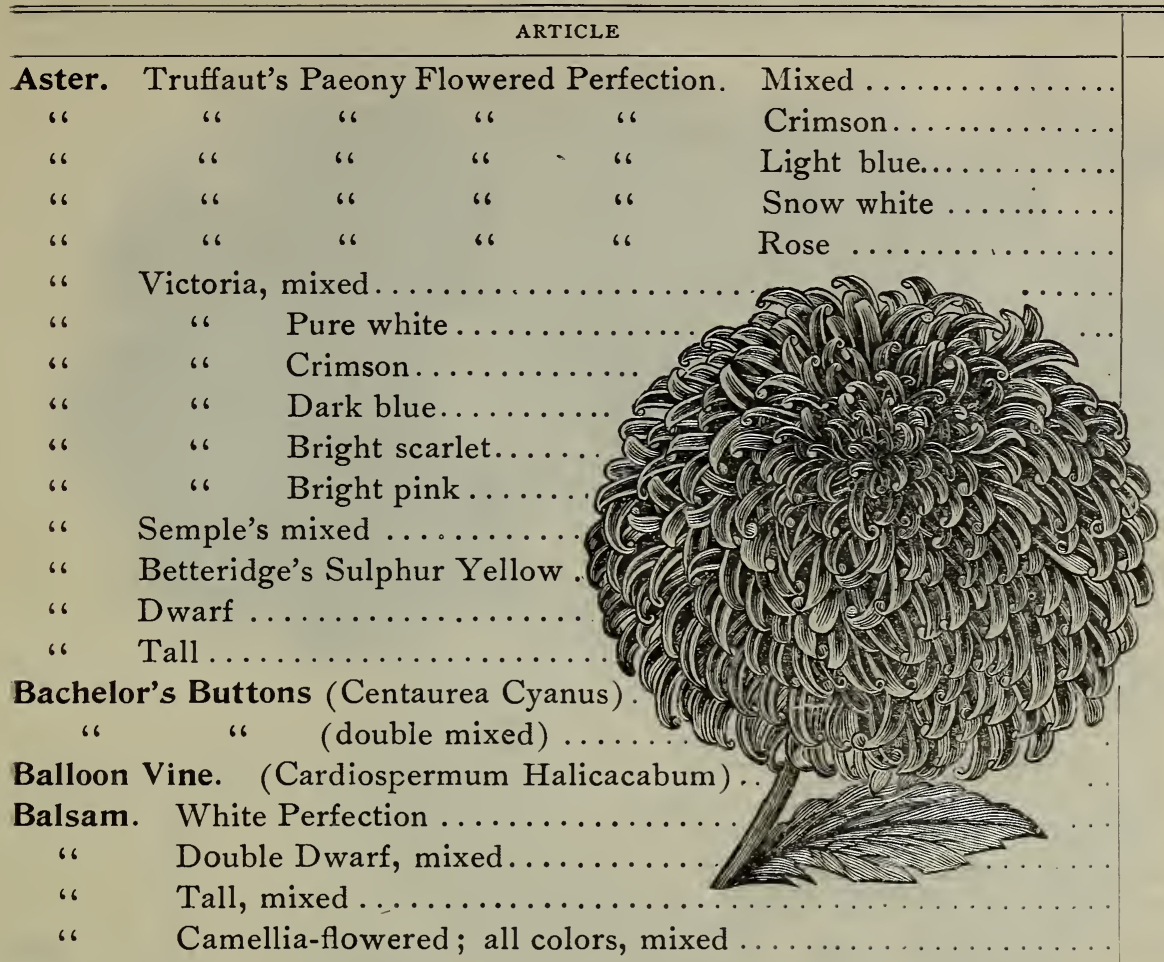

OZ.

1.00

1.25

1.25

1.25

1.25

1.25

2.00

2.00

2.00

2.00

2.00

1.00

.60

.50

.50

.10

.20

.10

.50

.35

.35

.40

.15

Bartonia Aurea

(Momordica)

.10

Begonia. Tuberous rooted, finest single, mixed........ $\frac{1}{16}$ oz., $\$ 3.50$

Vernon, per oz., $\$ 5.00 ; \frac{1}{16}$ oz., .50.

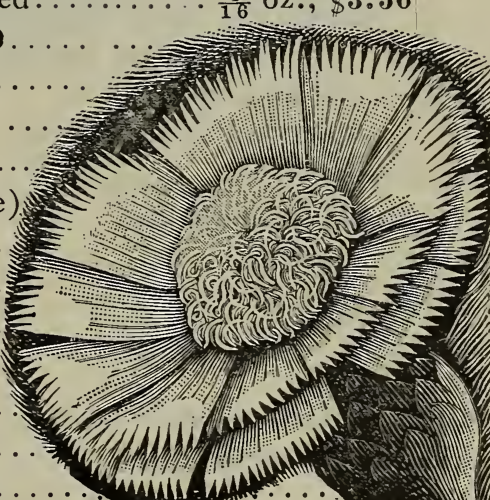

Canna. Crozy's finest hybrids, mixed... .........

Canterbury Bell. (Campanula Medium). Single, mixed ..........

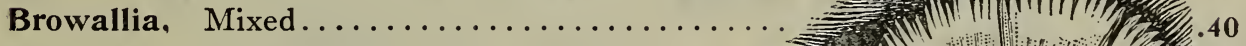

Calliopsis Fine mixed.................

Calendula. Mixed ................... Canary Bird Flower. (Tropaeolum Canariense) $\div$ Candytuft. White......................

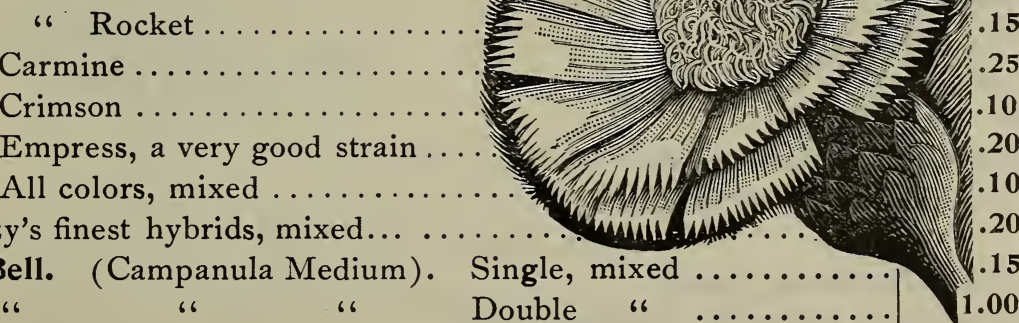

، 6 6 6 6 Double "6 ......... 1.00

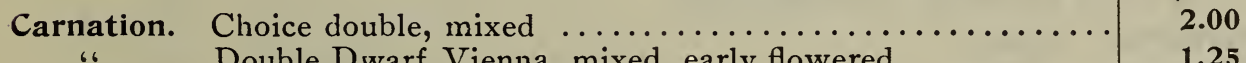

، Double Dwarf Vienna, mixed, early flowered........... 1.25

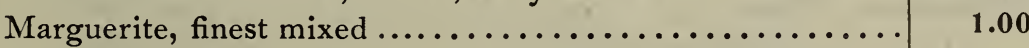

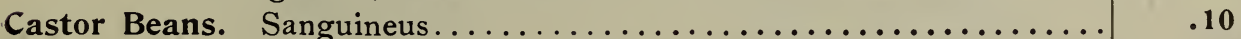

.10

Borboniensis............................... $\quad \begin{array}{r}10 \\ \quad 10\end{array}$

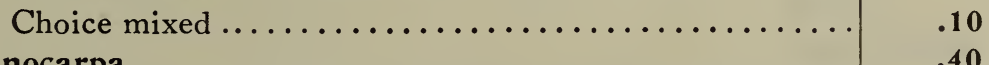

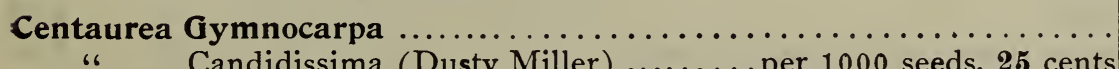

1.00

.15

1.00

66

Cyanus, Emperor William, blue.

New Margaret
12.00

16.00

5.00

5.00

1.00

2.00

1.00

6.00

3.50

3.50

5.00

1.75

.90

5.00

.80

.60

3.00

.50

1.00

2.50

1.00

1.50

.70

2.00

1.50

10.00

14.00

.40

.60

.60

.40

$4 \cdot 00$

1.00 


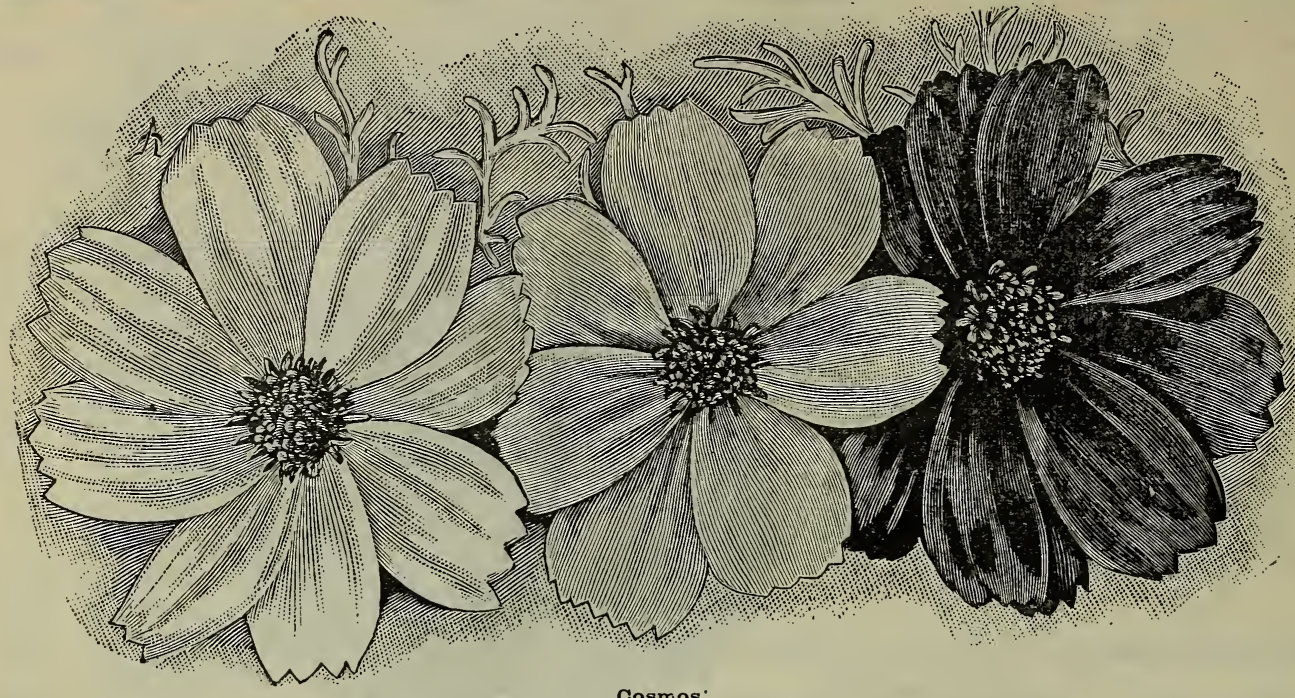

Cosmos:

ARTICLE.

Cockscomb. Cristata, tall sorts mixed..................

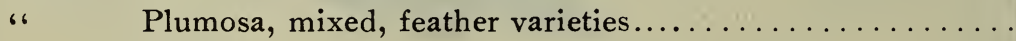

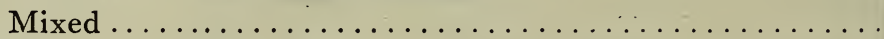

Chrysanthemum. Carinatum, mixed.

annual varieties

" yellow

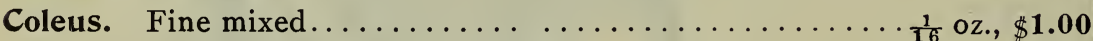

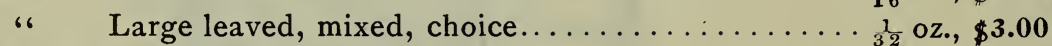

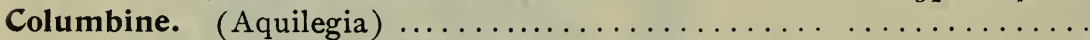

Convolvulus Minor. Mixed (Dwarf Morning Glory) $\ldots \ldots \ldots \ldots \ldots \ldots$

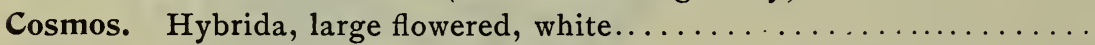

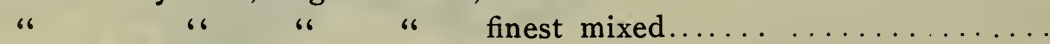

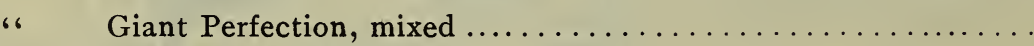

، New Early Flowering Hybrids, mixed ................

Cuphea Purpurea. Mixed............................

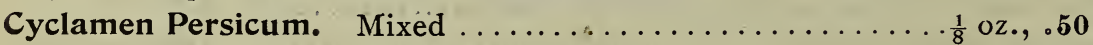

" " Giganteum, mixed ............ $\$ \frac{1}{8}$ oz., $\$ \mathbf{2 . 0 0}$

Cypress Vine. Scarlet (Ipomea Quamoclit) $\ldots \ldots \ldots \ldots \ldots \ldots \ldots \ldots$

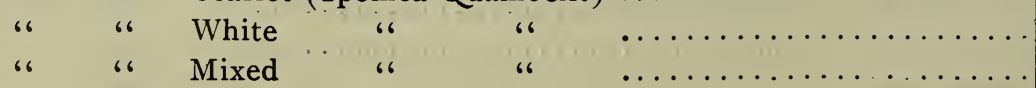

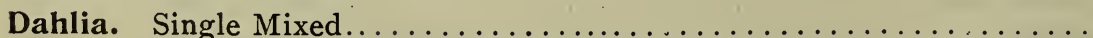

6 Finest double mixed, from selected flowers $\ldots \ldots \ldots \ldots \ldots \ldots$

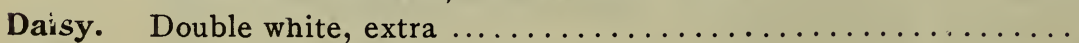

" " red, (Longfellow) finest strain $\ldots \ldots \ldots \ldots \ldots \ldots \ldots$

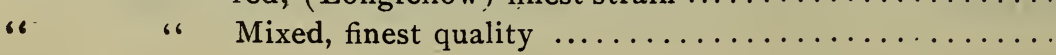

$\mathrm{oz}$.

.60

.50

.50

.15

.15

.15

.15

.10

.20

.10

.10

.50

7.00

LB.

3.00

.10

1.00

1.00

1.00

1.00

.75

1.50

.75

.75

5.00

.30

.30

.20

3.00

.60

2.00

.50

.40

3.00

.15

1.25

.20

1.50

.15

1.25

.25

3.00

.75

7.50 


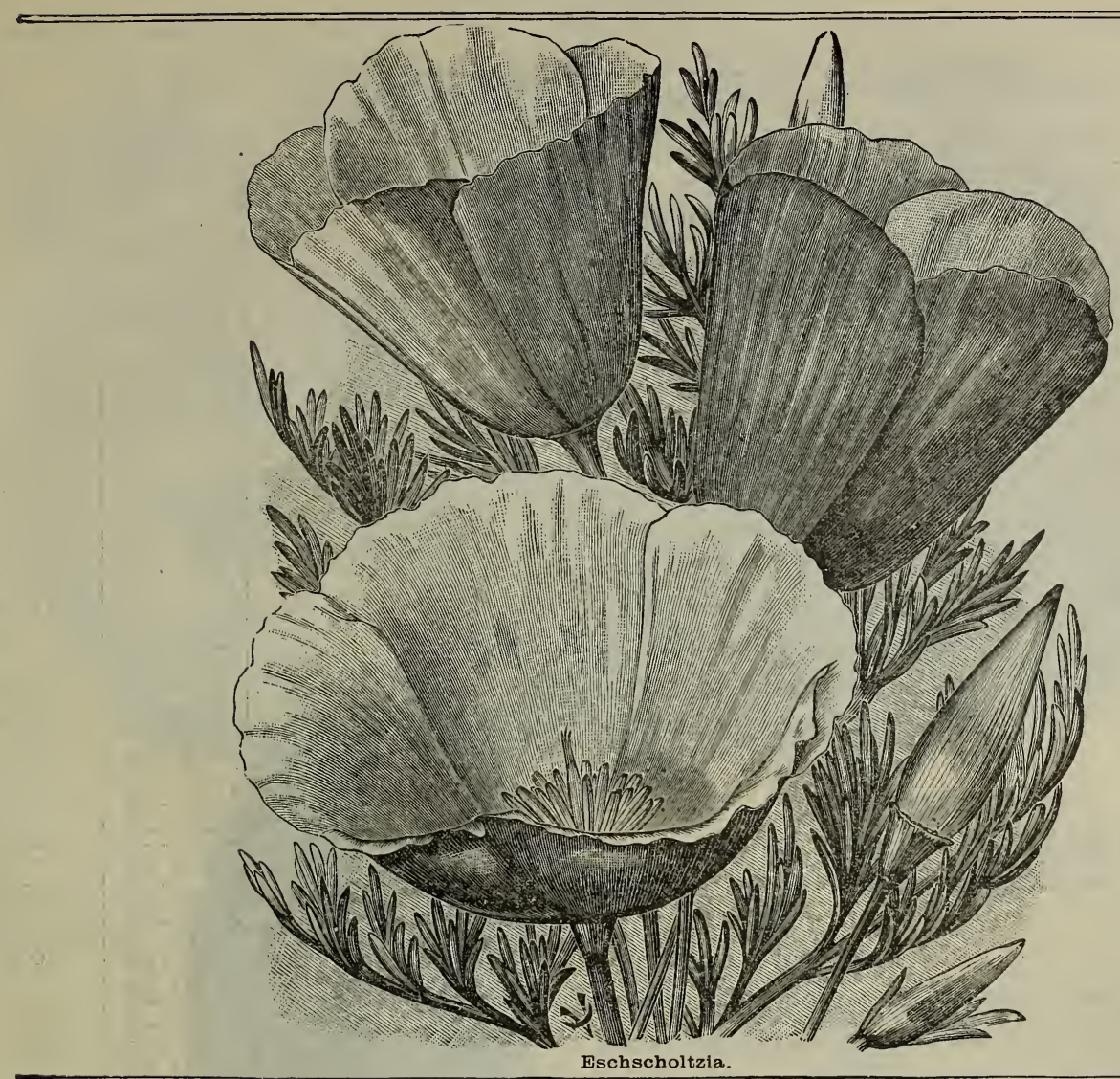

\begin{tabular}{c|c}
\hline ARTICLE. & Oz.
\end{tabular}

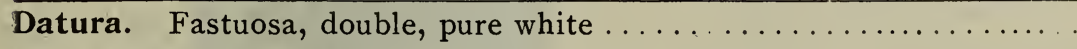

Eschscholtzia. (California Poppy), mixed, fine colors..............

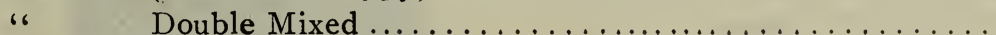

Freezia. Refracta Alba............................

oz.

.20

Forget $=$ Me $=$ Not.

66

66

6

(Myosotis Alpestris), Blue

Alpestris, white

Victoria.

Mixed.
Four O'clock. (Marvel of Peru), mixed

Fox Glove. (Digitalis), splendid mixed

Globe Amaranth. (Gomphrena), mixed .

Godetia. Many sorts mixed ........................

Gourds. Dish Cloth ................................

، Japanese Nest Egg, (white, egg shaped) . . . . . . . . . . .

" Siphon or Dipper..........................

"6 Hercules Club

" Sugar Trough

66 Ornamental Pomegranate

66
.25

.60

.40

1.00

.50

.10

.15

.10

.10

.15

.10

.15

.15

.10

.15

.10

.25

.30

.75

3.00
LB. 


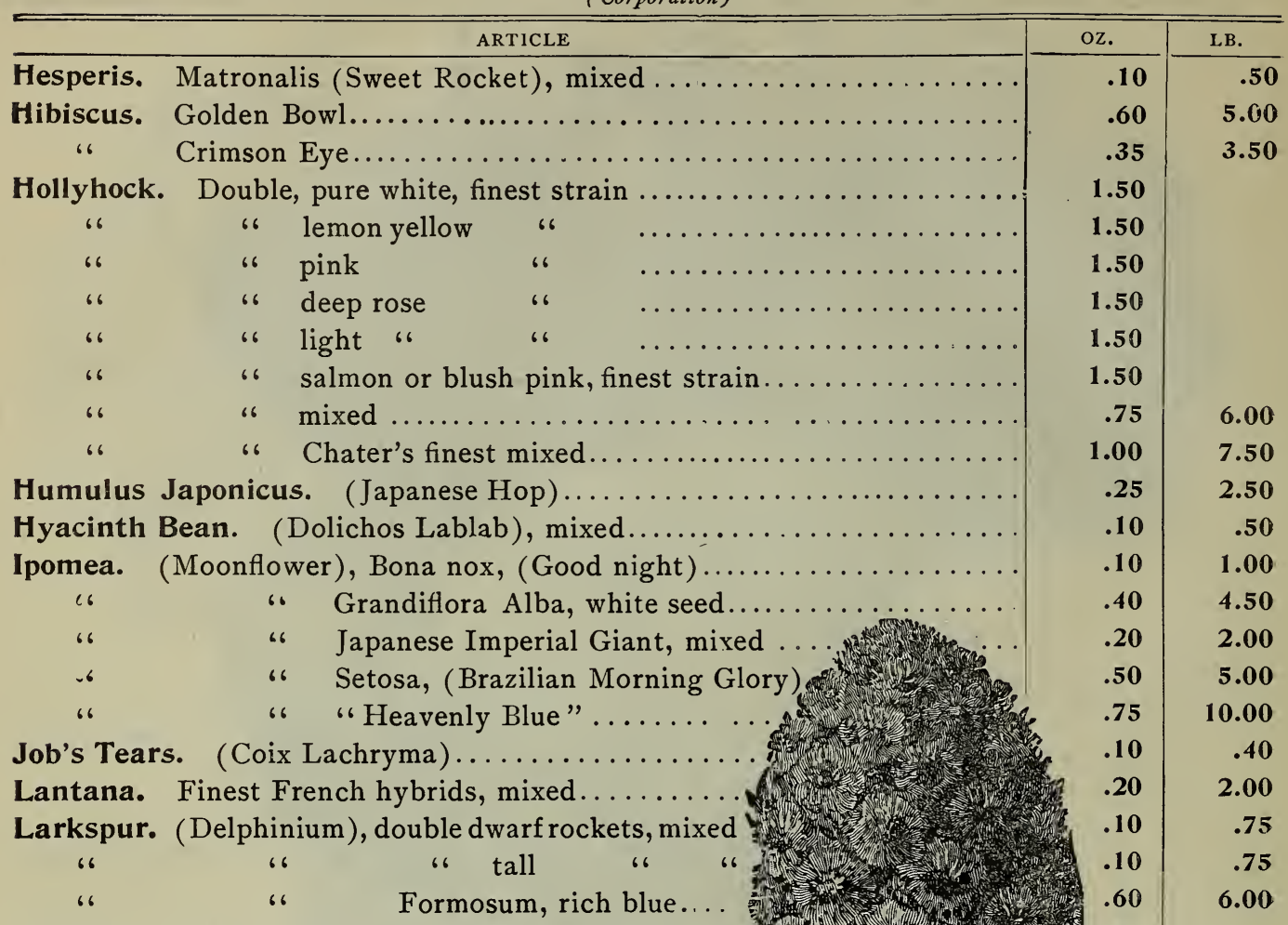

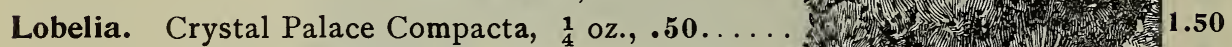

"6 Speciosa, dark blue flowers ...........

6 Erinus, Emperor William, ${ }_{4}^{1}$ oz., .45 ....

، "6 (Gracillis), blue.............

Linum. Grandiflorum Rubrum ..............

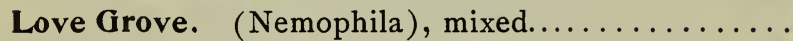

Lupinus. Annual sorts, mixed, tall varieties.......

Marigold. Meteor, (Calendula Officinalis) .......

، Prince of Orange ، $\ldots \ldots \ldots \ldots$.

"6 African, double mixed................

6 6 6 Eldorado..................

"6 " 6 French Legion of Honor .........

6 6 " Tagates Signata Pumila, (yellow)..

6 ، ، Double Dwarf French, mixed .....

Matricaria. Capensis, fl. pl., double white Feverfew.

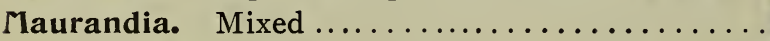
Mesembryanthemum. Crystallinum, (Ice Plant).

Mignonette. Sweet, (Reseda Odorata Grandiflora).

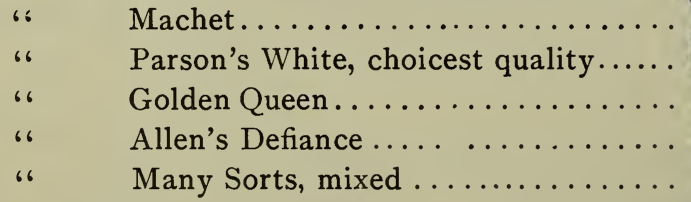

Mimosa. Pudica. Sensitive Plant............

Mimulus. Moschatus (Musk Plant) ...........

" Tigrinus, mixed.............. Mignonette.

4.25

4.25

.65

.60

.50

.60

.60

1.75

2.50

2.50

3.00

1.25

2.25

1.50

.50

5.00

2.00

5.00

2.00 


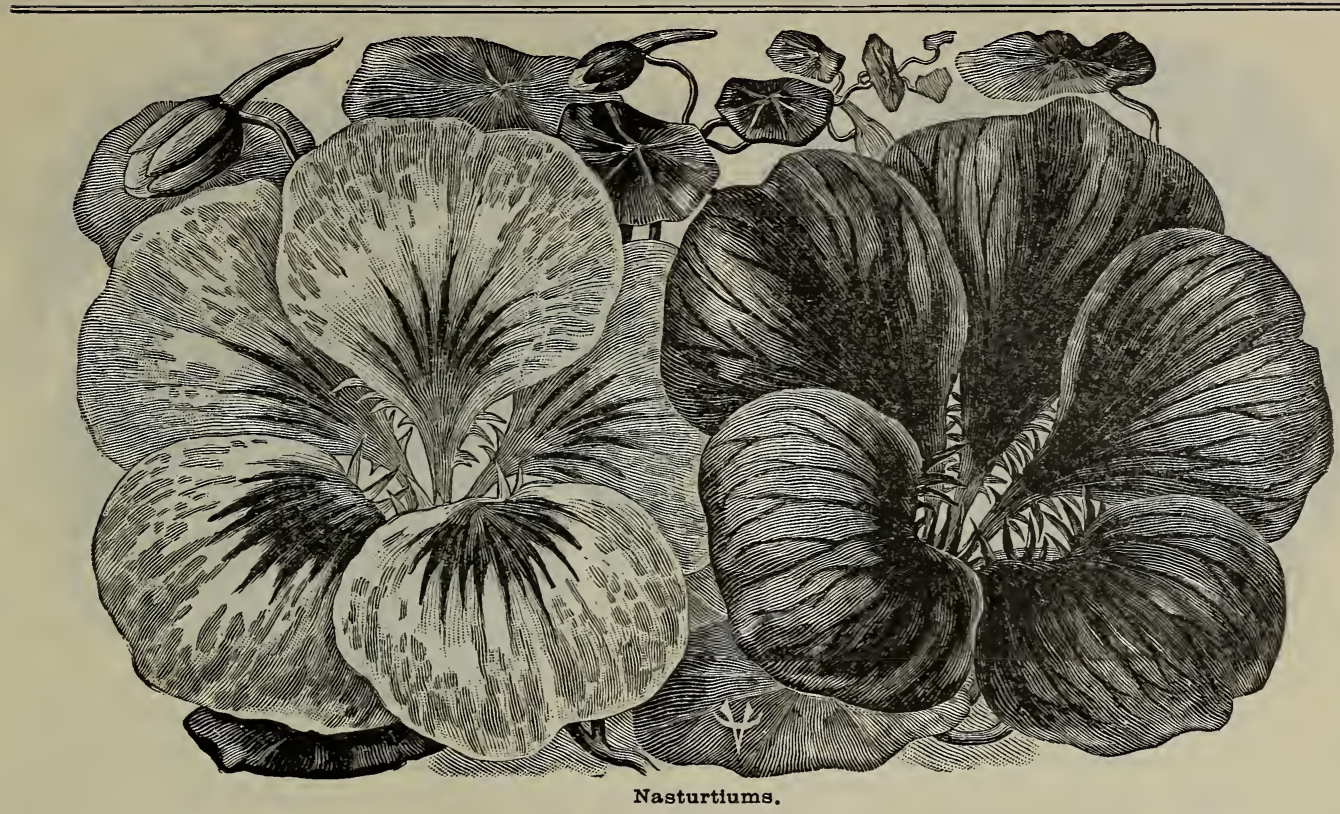

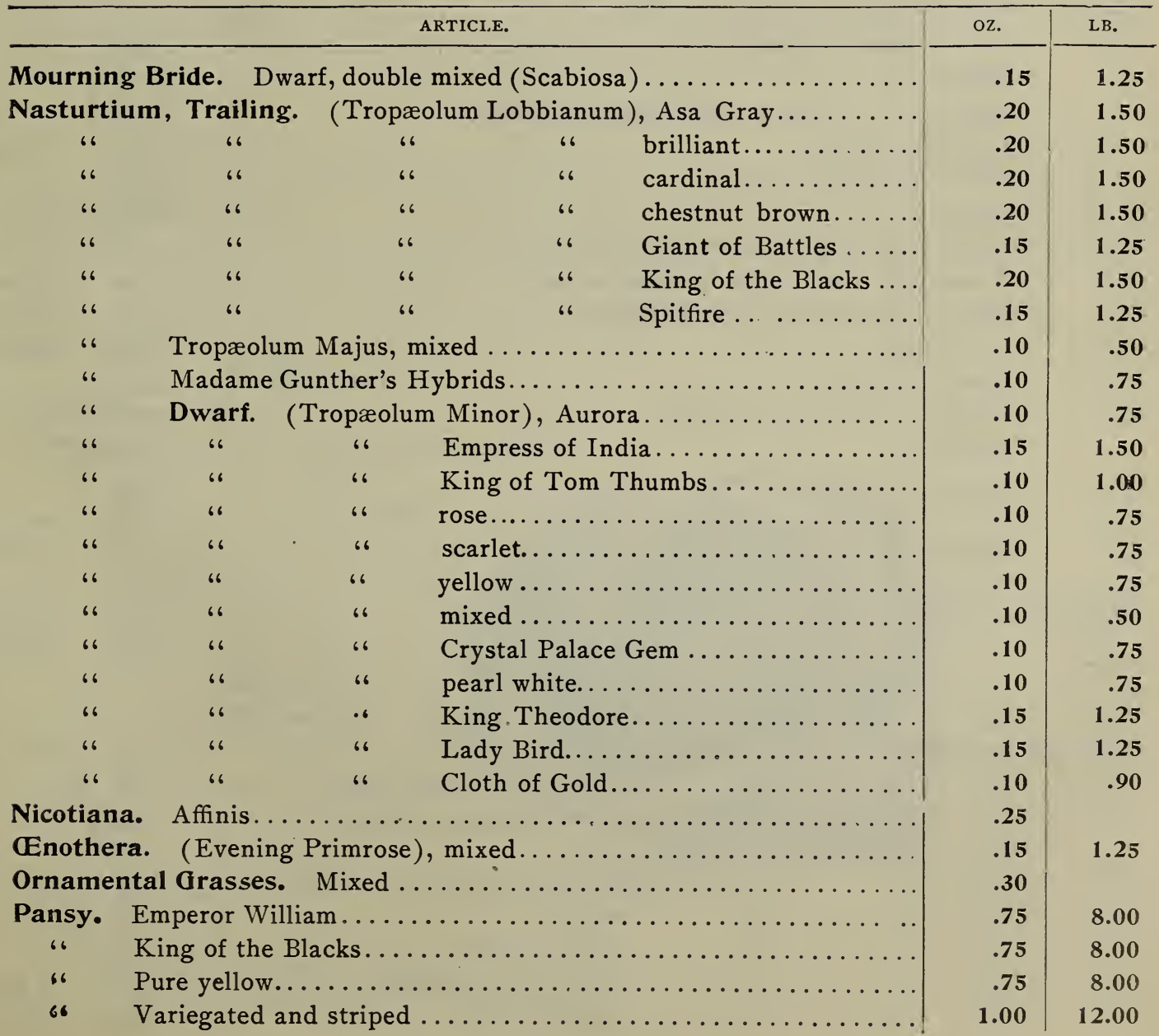




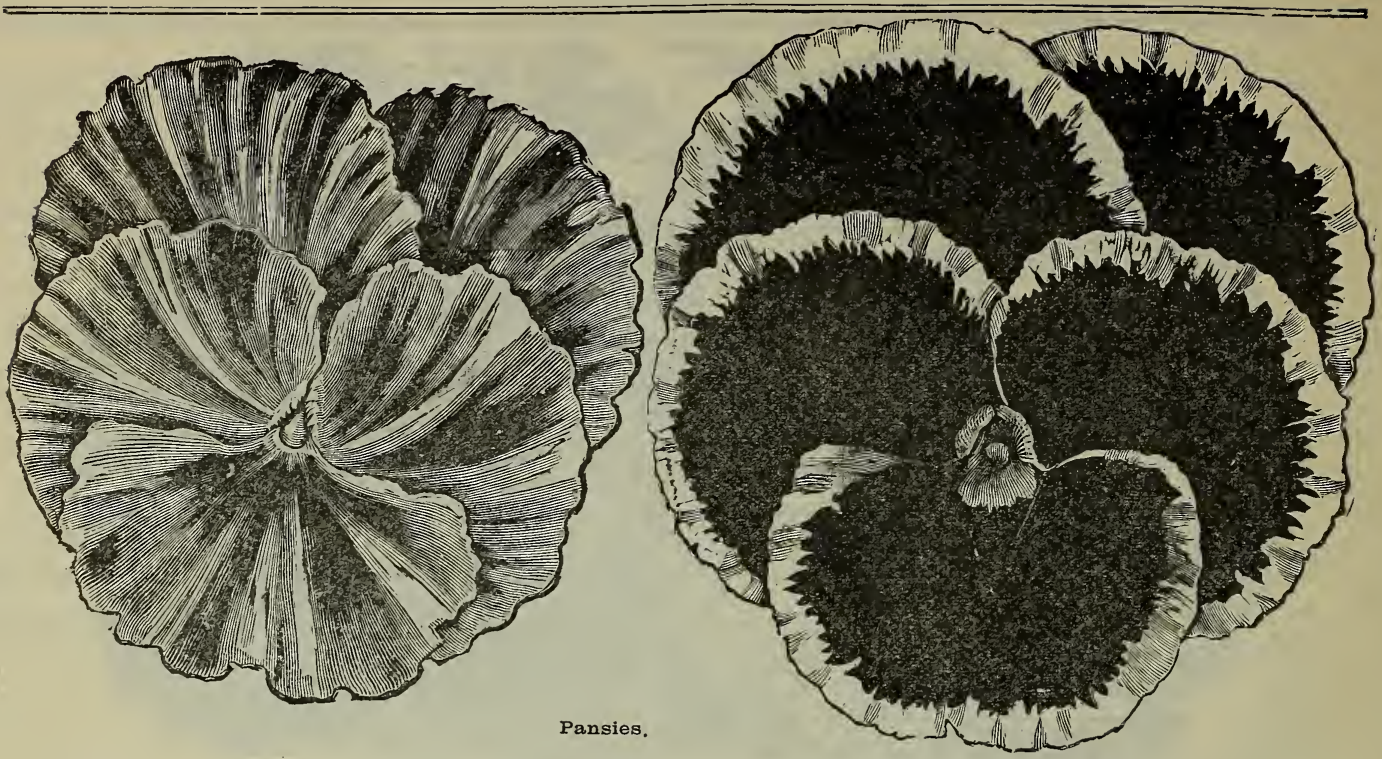

\begin{tabular}{|c|c|}
\hline & ARTICLE. \\
\hline ns.y. & Odier or large stained, extra fine.................. \\
\hline 66 & 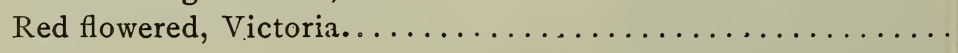 \\
\hline 66 & 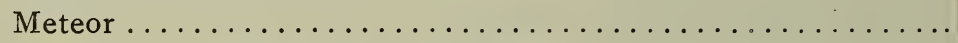 \\
\hline 66 & Bugnot's very large stained, mixed. . . . . . . . . . . . . \\
\hline 66 & 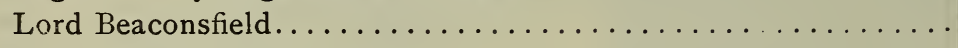 \\
\hline 66 & 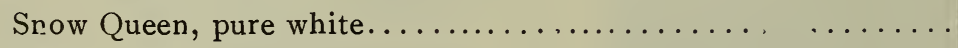 \\
\hline 66 & 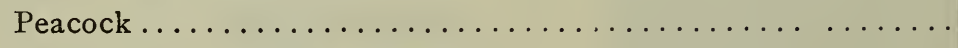 \\
\hline 66 & Large Flowering Parisian, large stained . . . . . . . . . . . \\
\hline 66 & Trimardeau, very large flowered, mixed $\ldots \ldots \ldots \ldots \ldots \ldots$ \\
\hline 66 & Extra choice mixed, large flowering $\ldots \ldots \ldots \ldots \ldots \ldots \ldots$ \\
\hline 66 & 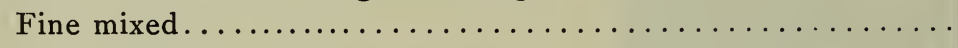 \\
\hline 66 & Giant $\operatorname{mixture} \ldots \ldots \ldots \ldots \ldots \ldots \ldots \ldots$ Per $_{4}^{1}$ oz., $.9 \ldots$ \\
\hline 66 & Breck's International, mixed $\ldots \ldots \ldots \ldots \ldots \ldots \ldots \ldots \ldots \ldots \ldots \ldots \ldots \ldots$ \\
\hline "6 & 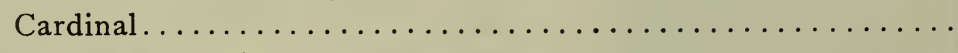 \\
\hline 66 & 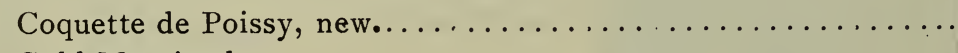 \\
\hline 66 & Gold Margined $\ldots \ldots \ldots \ldots \ldots \ldots \ldots \ldots \ldots \ldots \ldots \ldots$ \\
\hline 66 & Quadricolor, (Pheasant's Eye) $\ldots \ldots \ldots \ldots \ldots \ldots \ldots$ \\
\hline 66 & $\ldots \ldots \ldots \ldots \ldots \ldots \ldots \ldots$ \\
\hline
\end{tabular}

oz

3.50

1.50

1.00

6.00

1.00

12.00

1.25

3.00

1.75

1.25

16.00

1.00

.50

3.00

6.00

1.50

1.50

.75

.75

.75

1.00

.50

9.00

9.00

8.00

Petunia. Hybrida, fine striped and blotched

12.00

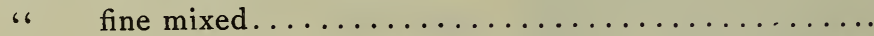

${ }_{16}^{1}$ oz. 1.50

، large flowered, choicest mixed.

large flowered, extra double, fringed..... $\frac{1}{32}$ oz. 5.00

Phlox Drummondi. (Large flowered), Alba, pure white.............

\begin{tabular}{|c|c|c|}
\hline ، & ، & Isabellina, cream yellow . \\
\hline ، & ، & Kermesina Alba, aculata \\
\hline 6 & ، & 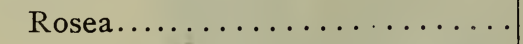 \\
\hline & 66 & Splendens, crimson (pure white ey \\
\hline 66 & ، 6 & scarlet $\ldots \ldots \ldots \ldots \ldots \ldots$ \\
\hline & 66 & Black Warrior........ . . \\
\hline 8 & ، 6 & extra, choicest mixed...... \\
\hline & 66 & splendid mixed \\
\hline
\end{tabular}




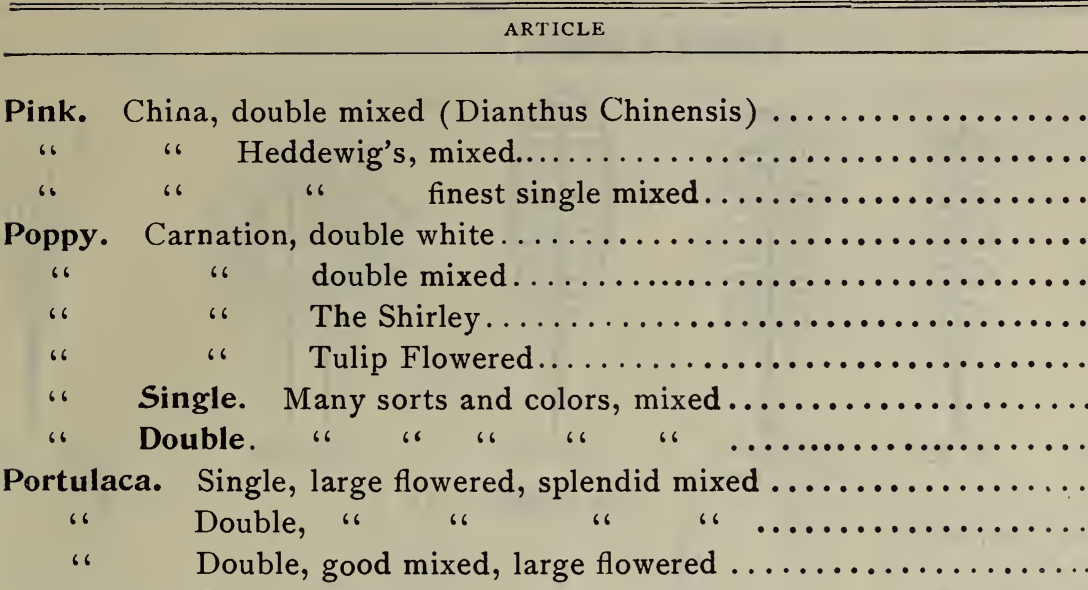

Primula. Sinensis Fimbriata, choicest mixed ..........

Pyrethrum. Parthenifolium, Aureum (Golden Feather)............. 66 Roseum .

Rhodanthe. Mixed

Salpiglossis. Extra fine mixed hybrid varieties.................

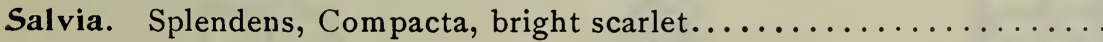

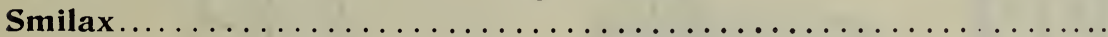

Snapdragon. (Antirrhinum), finest mixed

Stock. Double German Ten Weeks, mixed.

.60

.40

.15

.10

.30

.60

.15

.15

.20

4.00

2.00

20.00

.40

.75

.60

.40

2.50

.35

.25

2.00

$2.5($ 。

1.25

1.75

.15

.15

.15

.10

.15

.30

.50

1.00

.60

.65

.70

.65

1.00

1.00

.50

1.00

.60

.85

1.50

.15

.20

.20

.40

.25
2.00

.600

4.00

1.00

.60

3.00

6.00

1.25

1.25

2.25

4.00

4.00

3.50

2.50

1.50

1.00

1.25

.75

1.00

3.00

4.00

6.50

6.50

7.00

6.50

12.00

10.00

5.00

14.00

7.00

10.00

1.50

1.50

2.00

4.00

2.50 


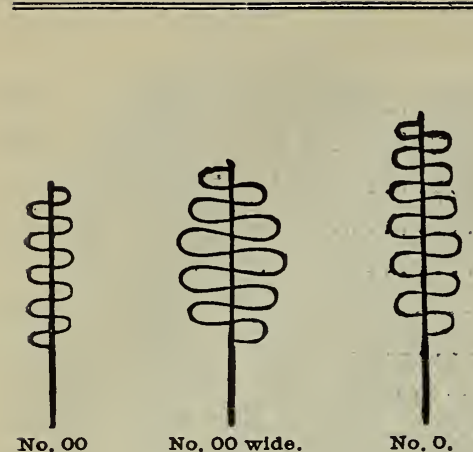

No. $\infty$, I8-in. stick, $3^{1 / 2}$ in. wide

" oo wide, 20 -in. stick, 8 in. wide " o, 24-in. stick, 5 in. wide

" o wide, 24-in. stick, 8 in. wide " $\mathrm{o}^{1 / 2}, 24$-in. stick, $7^{1 / 2}$ in. wide

Ivy Trellis. - 16 in. diameter 20 "."

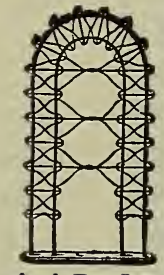

Arch Top Ivy.

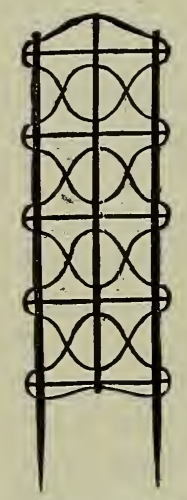

Veranda Trellis ARCH TOP IVY.
$28 \times$ I4 in., black walnut base .

BOW TRELLIS.

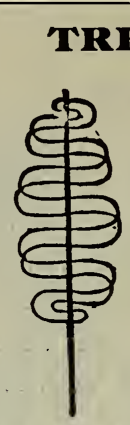

No. 0 wide. Each. Per doz.

\$0.05\$0.50

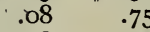

$.08 \quad .75$

. IO 1.00

$\begin{array}{ll}. \mathrm{I} 5 & \mathrm{I} .5 \mathrm{O}\end{array}$

$.50 \quad 5.00$

$.60 \quad 7.00$ $.75 \quad 9.00$

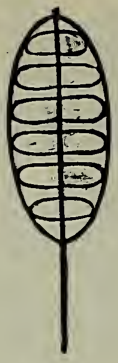

No. $01 / 2$.

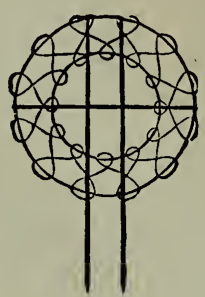

Ivy Trellis.

Discount $25 \%$.

Fan Trellis. - $\mathrm{I} / 2 \mathrm{ft}$.

\begin{tabular}{|c|c|c|c|}
\hline " & $2^{1 / 2}$ & "1 & \\
\hline 6 & $21 / 2$ & 6 & \\
\hline 66 & $3 \frac{1}{2}$ & " & $x$ \\
\hline 6 & 4 & "6 & $\mathbf{x}$ \\
\hline "6 & 5 & 6 & $\mathbf{x}$ \\
\hline 66 & 6 & "6 & $\mathbf{x}$ \\
\hline 6 & 7 & "6 & $\mathbf{x}$ \\
\hline "6 & 8 & 6 & $x$ \\
\hline
\end{tabular}

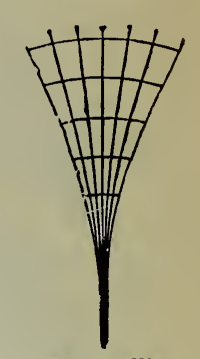

Fan Trellis. Each. Per doz. $\$ 0.10 \$$ I. $\$$

.I5 I.5O

$.20 \quad 2.00$

$.50 \quad 5.00$

$.50 \quad 6.00$

$.75 \quad 8.00$

.759 .00

I. 00 II 00 I.00 I2.00

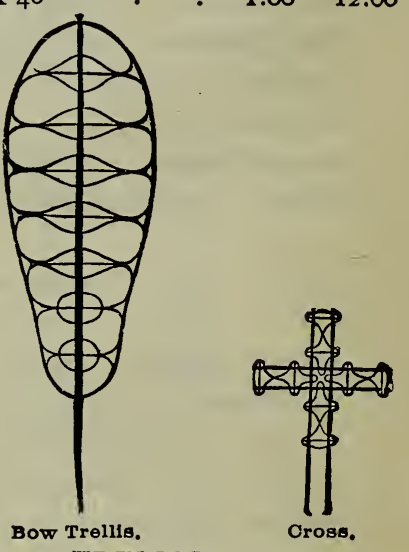

VERANDA TRELLIS.

\begin{tabular}{|c|c|c|c|c|c|c|c|c|c|c|}
\hline \multirow{3}{*}{$\begin{array}{l}\text { Each. } \\
\$ 1.00\end{array}$} & \multirow{3}{*}{$\begin{array}{r}\text { Per doz. } \\
\$ 10.00\end{array}$} & \multirow{2}{*}{\multicolumn{8}{|c|}{ VERANDA TRELLIS. ${ }_{\text {Each. }}$}} & \multirow{2}{*}{$\begin{array}{r}\text { doz. } \\
\$ 3.00\end{array}$} \\
\hline & & & & & & & & & & \\
\hline & & 3 & “" & & & & . & . & .40 & \\
\hline$\$ 0.20$ & $\$ 2.00$ & 4 & “" & & & & . & . & .50 & 5.00 \\
\hline .30 & $3 \cdot 50$ & $5 \times 11 / 2$ & " . & & & & . & 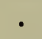 & .70 & 8.00 \\
\hline .40 & 4.00 & $6 \times 11 / 2$ & “. & & & & 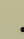 & . & .80 & 9.5 \\
\hline .45 & 4.50 & $7 \times 1 \frac{1}{2}$ & “" & & & & & $\cdot$ & .95 & 1.50 \\
\hline $\begin{array}{l}.50 \\
.60\end{array}$ & $\begin{array}{l}5.00 \\
6.50\end{array}$ & $8 \times 11 / 2$ & " : & & & & & . & I.10 & 3.00 \\
\hline .75 & 8.00 & $9 \times 12 / 3$ & “. & & & & & - & I. 25 & 5.0 \\
\hline & & IO $\times \mathrm{I}^{2} / 3$ & " . & & & & & . & I. 50 & 18.0 \\
\hline$\$ 0.30$ & $\$ 3.00$ & I I $\times 2$ & “ & & & & & . & I. 75 & 21.00 \\
\hline .40 & 4.50 & $12 \times 2$ & “ & & & & & 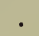 & 2.00 & 24.0 \\
\hline
\end{tabular}

PLANT STICKS, ROUND or SQUARE, Painted Green.

\begin{tabular}{|c|c|}
\hline $4 \mathrm{ft} . \times 0 . / 16 \mathrm{in}$ & 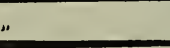 \\
\hline $3 / 2 \mathrm{ft} . \times 2 / 15$ in. & . \\
\hline $3 \mathrm{ft} . \times 1 / 2 \mathrm{in}$ & . \\
\hline $2 \%$ ft. $x$ / $/ 16$ in. & \\
\hline $2 f t . \times 3 / 8$ in. & $=$ \\
\hline $11 / 2 \mathrm{ft} .+5 / 16 \mathrm{in}$ & 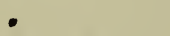 \\
\hline
\end{tabular}

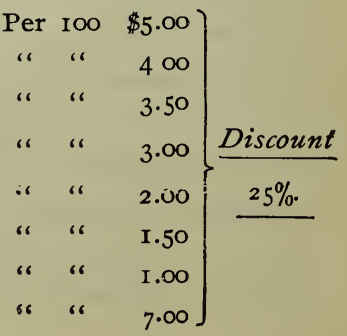




\section{GARDEN REQUISITES, ETC.}

\section{FLO WER-POTS, SAUCERS, and SEED-PANS.}

Inside Measurement, width and depth equal. Packing charged extra.

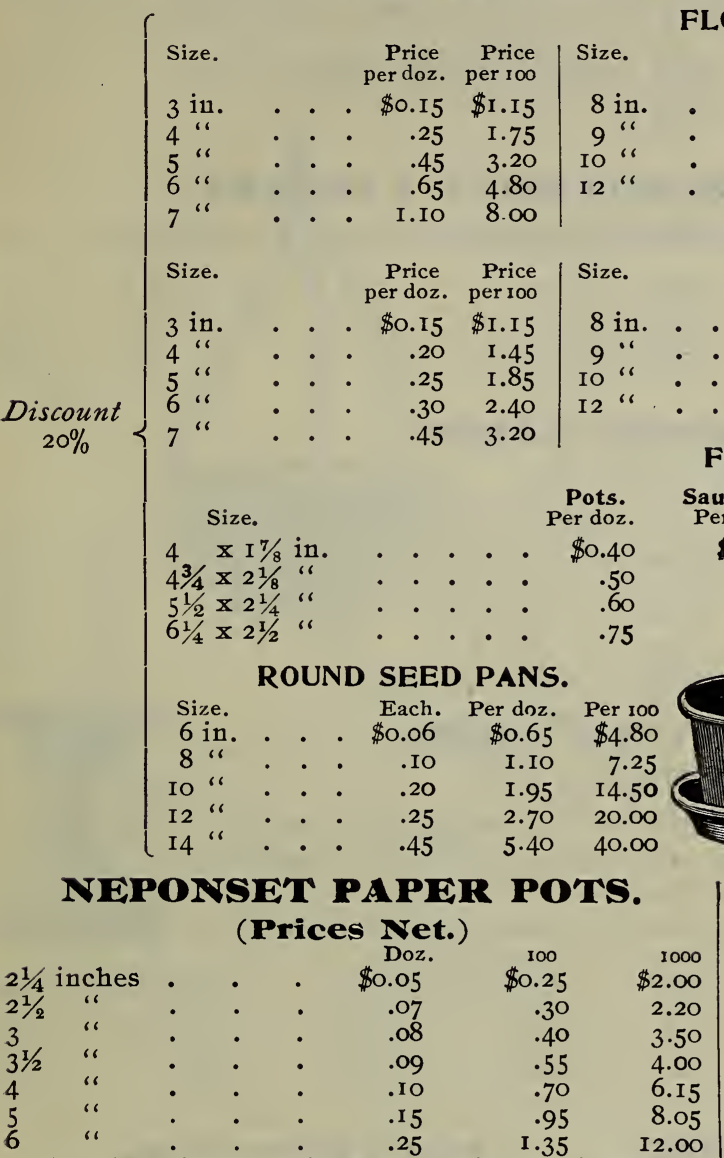

Not less than one thousand at thousand rate.

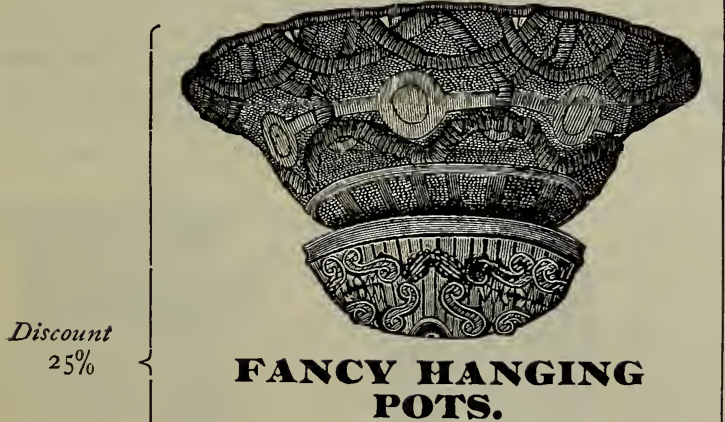

We carry a full line of these goods and can furnish them in sizes varying from 6 to 12 inches in diameter and at prices ranging from 20 cents to $\$ 2.00$ each, according to size and pattern.

\section{BRASS JACK CHAINS.}

For Hanging Pots. No. I I 8,3 strand, doz., $\$ 2.25$; No. II9, 4 strand, doz., $\$ 2.25$.

FLOWER POTS.

\section{FERN PANS.}

\begin{tabular}{rr|lrr}
$\begin{array}{c}\text { Price } \\
\text { per doz. price }\end{array}$ & Size. & $\begin{array}{c}\text { Price } \\
\text { per doz. }\end{array}$ & Price \\
per 100
\end{tabular}

$\mathrm{I}_{3}$ in. . . . $\$ 8.00 \$ 60.00$

$\$ 1.50 \$ 11.25$

$2.15 \quad 16.00$

$2.90 \quad 21.50$

Each.

14 " . . . \$0.90

I5 " $\cdots \cdot$ - 1.20

SAUCERS. \begin{tabular}{cc|lcr}
$\begin{array}{c}\text { Price } \\
\text { perdoz. }\end{array}$ & $\begin{array}{c}\text { Price } \\
\text { per } 100\end{array}$ & Size. & $\begin{array}{c}\text { Price } \\
\text { per doz. }\end{array}$ & $\begin{array}{c}\text { Price } \\
\text { per } 100\end{array}$
\end{tabular}

$\$ 2.15 \$ 16.00$

Each.

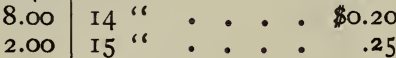

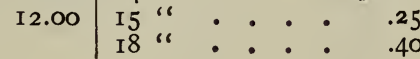

\begin{tabular}{c|cc} 
Pacers. & $\begin{array}{c}\text { Pots. } \\
\text { Perdoz. }\end{array}$ & Saucers. \\
Per doz.
\end{tabular}

$\$ 0.257 \times 2 \frac{5}{8}$ in. . . . . \$o.85 \$0.50

$.30 \quad 8 \times 31 / 8$ " . . . . $1.25 \quad .75$

$.40 \quad 9 \times 3 \frac{1}{4} "$. . . . . $1.50 \quad 1.00$

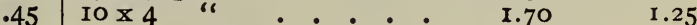

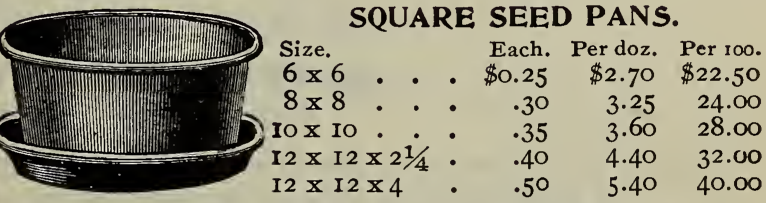

PATENT EXTENSION

HANGER.

For Pots and Bird Cages. Brass, dozen, $\$ 2.25$.

\section{CEDAR PLANT TUBS.}

For growing Agaves, Century and other large plants. Made of the best red cedar; painted green; iron handles.

No. I. Dia. $28 \times 22$ deep, each, $\$ 5.00$

"2. " 26 × 20 " 24 " 4.25

"3. " $324 \times 181 / 2$ " $24 \quad$ " 3.50

"4. " $422 \times 17$ " " 2000

" 5 " " 20 X 16 " 6 " 2.50

"6. " " $6181 / 2 \times 15$ " " 1500

" 7 " " $161 / 2 \times 14$ " 140 "

"8. " $161 / 2 \times 13$ " 1300

\section{OAK PLANT TUBS.}

Natural Wood finish. These are strong, light, and thoroughly serviceable.

I2 in. diameter, II in. high . . \$I.I5 I4" " $"$ I2" " I6" " 6 I3" $"$ " . . I.65 I8 " " 6 I5 " 20 " " 17 " 17 " . . . 2.25 24 " " 20 " " . . . 2.65

\section{CYPRESS PLANT TUBS.}

Made of Cypress, strongly hooped, iron handles, iron or wooden feet, durable and ornamental.

No. I. Dia. I3 x I I deep, each, \$o.75

"2. " $141 / 2 \times 13$ " 3 " 1.25

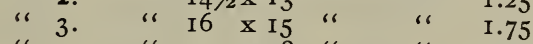

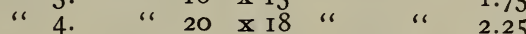

“ 5 .
Discount $5 \%$ 


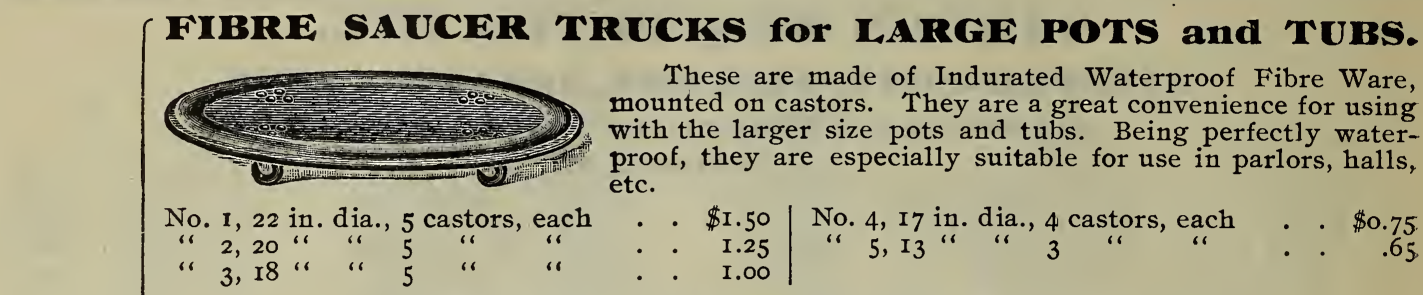

Discount $25 \%$

Discount $20 \%$

\section{FIBRE SAUCERS WITHOUT CASTORS.}

Made from Indurated Fibre Ware, prevents moisture passing through to injure table, floor or carpet.

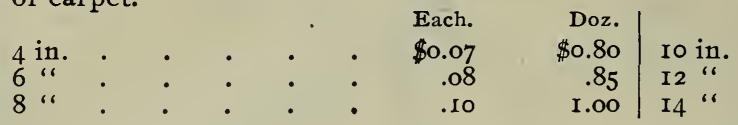

\section{FLORISTS' VASES.}

For Cut Flowers.

Made of Indurated Fibre Ware, light, neat and clean.

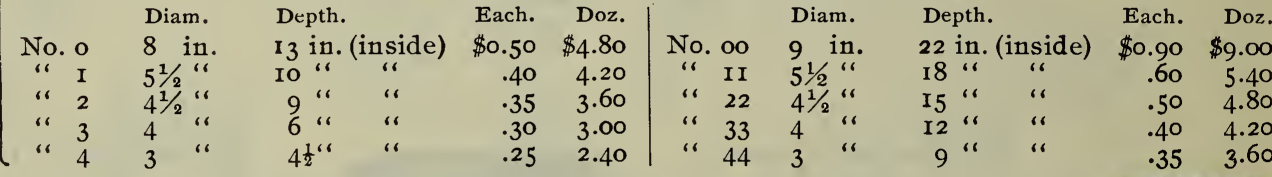

\section{BERLIN RESERVOIR VASES.} FOR LAWNS, CEMETERIES, ETC.

These iron vases are so constructed that one watering will keep the earth moist for a long time without souring the soil or injuring the roots. The reservoir is below the soil and water is taken up as wanted by capillary attraction.

Illustrations showing other designs and prices furnished upon application.

No. of $\begin{gathered}\text { Height, } \\ \text { Vase. Without Base. of Vase. Vase and Base. }\end{gathered}$ Beight of
Base.

$\begin{array}{lllll}\text { I } & \text { I9 } & 17 & 25 & \text { I } \\ 2 & 20^{1 / 2} & 201 / 2 & 28 & 1 \mathrm{I} / 2 \\ 3 & 29 & 27 & 36 & 2 \\ 4 & 33 & 35 & 45 & 4 \\ \text { BOUQUET TMOLIDERS } & \text { FOR }\end{array}$

These are indispensable for cemetery use, they are made of iron and nicely finished in green and bronze.

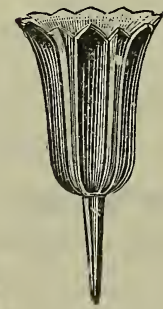

Tulip. Each. No. $4 \$ 0.20$ $\begin{array}{lll}\text { "1 } & 5 & 30 \\ \text { il } & 6\end{array}$

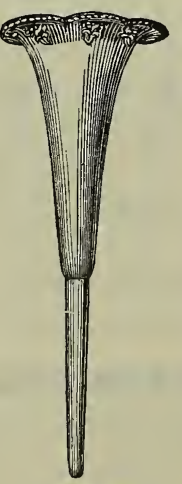

BluebelI.

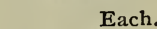

No. $2 \$ 0.30$

Cross $\quad .40$

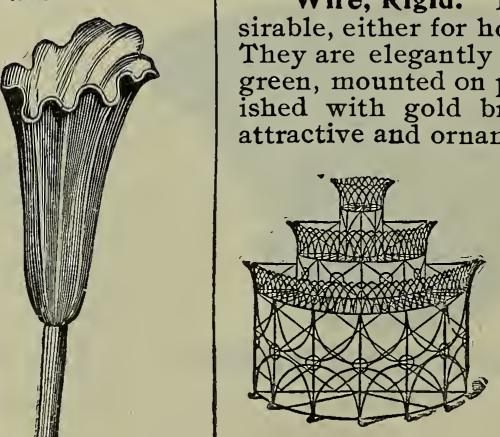

No. 4.

\begin{tabular}{|c|c|}
\hline \multicolumn{2}{|c|}{ 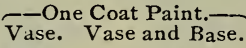 } \\
\hline $\begin{array}{r}\$ 4.00 \\
6.00\end{array}$ & \\
\hline 13.00 & I 5.00 \\
\hline 30.00 & 37.00 \\
\hline
\end{tabular}

PI,ANT STANDS. $\begin{array}{rr}\text { Each. } & \text { Doz. } \\ \text { \$O.I3 } & \$ 1.25 \\ .18 & 1.50 \\ .22 & 2.00\end{array}$ 

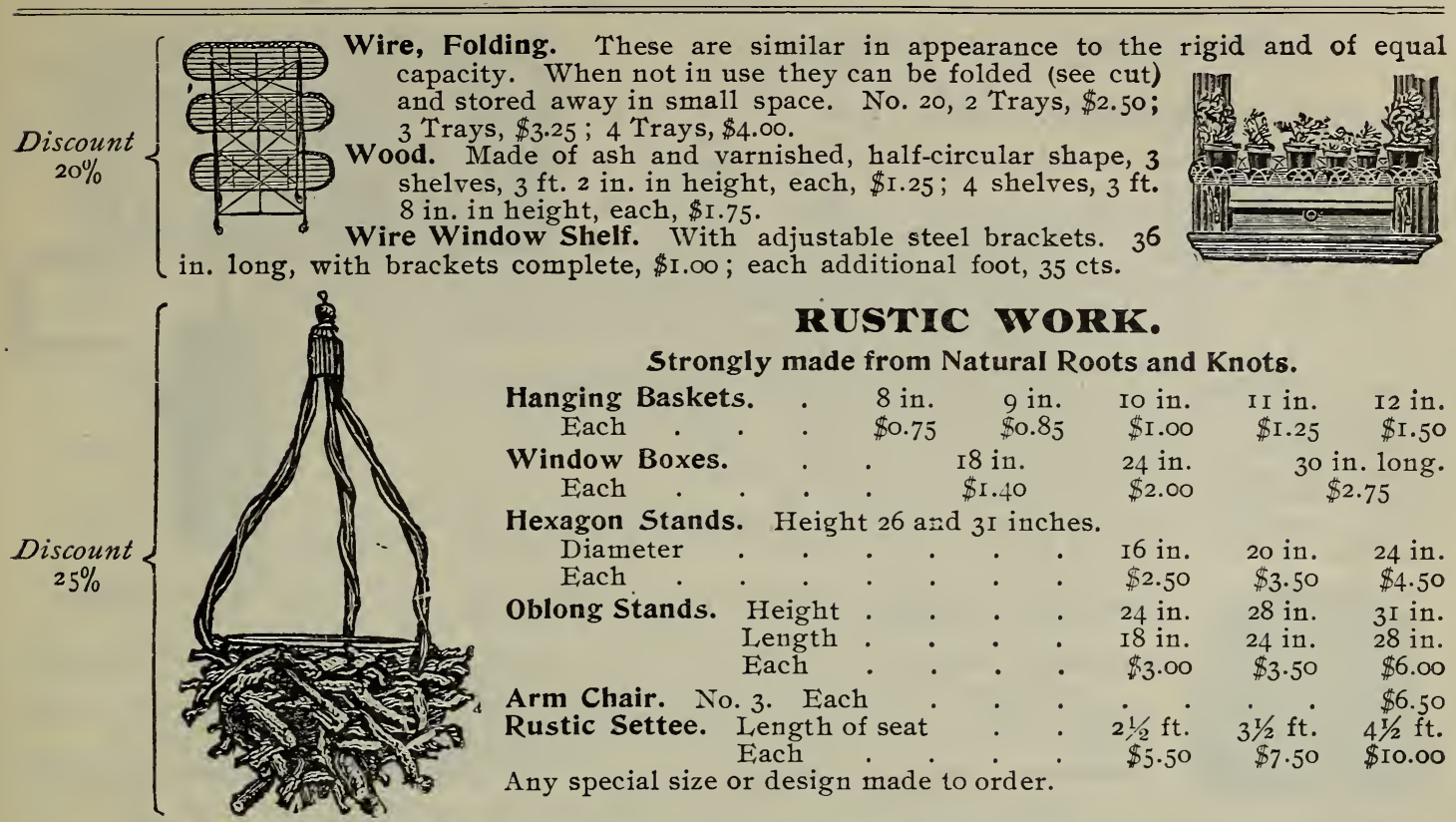

RUSTIC WORK.

Strongly made from Natural Roots and Knots.

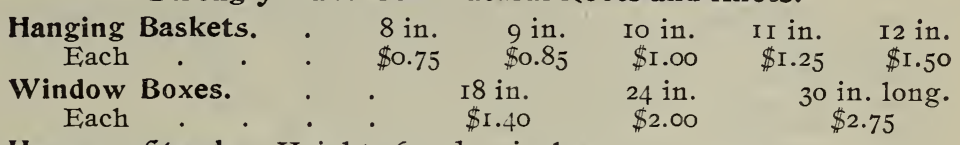

Hexagon Stands. Height 26 and $3 \mathrm{I}$ inches. Diameter Each

Oblong Stands. Height Each

Arm Chair. No. 3. Each

Rustic Settee. Length of seat Each

Any special size or design made to order.

I6 in. $20 \mathrm{in.} 24 \mathrm{in.}$ $\$ 2.50 \quad \$ 3.50 \quad \$ 4.50$ 24 in. 28 in. $3 \mathrm{I}$ in. I8 in. $24 \mathrm{in.} \quad 28 \mathrm{in}$. $\$ 3.00 \$ 3.50 \quad \$ 6.00$ ..$\quad \$ 6.50$ $2 \frac{1}{2} \mathrm{ft} . \quad 3 \frac{1}{2} \mathrm{ft} . \quad 4 \frac{1}{2} \mathrm{ft}$ $\$ 5.50 \quad \$ 7.50 \quad \$ 10.00$

\section{MISCELLANEOUS REQUISTTES.}

Baskets, Pansy or Verbena, Marston's Pattern. Wood or wire handles, standard size, ro in. 1ong, 5 in. wide, 3 in. deep, per I00, $\$ 1.80$; per I,000, $\$ 15.50$. Large size, I 2 in. long, 7 in. wide, 3 in. deep, per Ioo, $\$ 2.50$; per I,000, $\$ 22.00$.

Brushes, Flat Paint For applying tree ink, and general use. Thoroughly well made and of good material. $3 \frac{1}{2}$ in. wide, $\$ 2.00$ doz.

Caps, Hay, Breck's Improved. Brown, medicated, complete with ropes and pins, $45 \mathrm{cts}$. each; $\$ 40.00$ per roo.

Color, Improved Butter. Wells, Richardson \& Co.'s imparts the "golden tint of June" to butter at all seasons of the year. Bottles sufficient for 500 lbs., $\$ 2.25$ doz.; I, 2501 bs., $\$ 4.25$ doz.; 3,000 1bs., $\$ 8.50 \mathrm{doz}$.

Color, Hanson's Butter. A universal favorite. Bottles, same as above.

Cotton Batting. For packing flowers, etc., per bale, 6 doz. sheets, $\$ 2.75$.

Food, Breck's Flower. This preparation is perfectly colorless and clean to handle. It is the most concentrated, safest and effective plant food on the market. Soluble in water, and no trouble whatever to prepare for use. It promotes a healthy, vigorous growth of wood, leaf and flower. Put up in two sizes - beautiful lithographed boxes.

No. I, sufficient for 30 plants for one year, $\$ 2.00 \mathrm{doz}$.

No. 2, sufficient for 30 plants for 3 months, $\$ 1.00$ doz.

Flower Food, Bradley's Lily Brand. No. I, same as above.

Food, Imperial Liquid Plant. This preparation is said to contain all the food necessary for a healthy growth. It is in a liquid form and will be appreciated by those who grow plants iu the house. Per doz. pt., $\$ 2.25$; doz. qt., \$3.80.

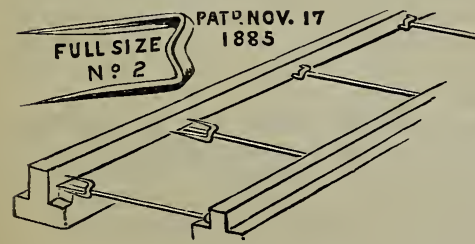

Glazing Points, Van Reyper, Perfect. The only durable and reliable points. Made of galvanized steel wire; no rights and lefts. Three sizes, Nos. $2,2 \frac{1}{2}$ and 3 . The Nos. 2 and $2 \frac{1}{2}$ are for general use ; the No. 3 for extra heavy work. Price, per box of $1,000,60$ cts. ; 5,000 and over, 55 cts. per I,000.

Krop Kollar, Faye's Patent. This is one of the simplest, yet most useful articles that has been offered the gardener and farmer for the protection of early crops from cut worms, cold winds and frosts. It consists of a flexible fibre-board collar that can be quickly adjusted around plants, a glass roof easily fastened on, and a shutter for use in extra

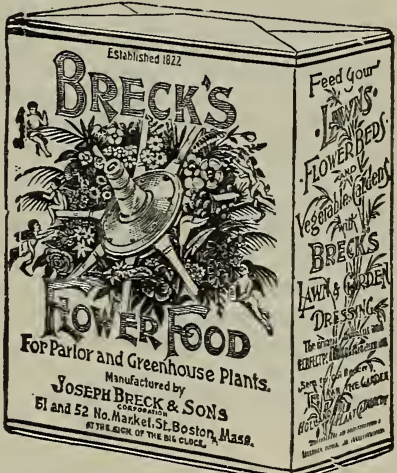
cold weather. We believe it will prove of great value for protecting corn, melons and other early or tender plants. $\$ 4.00$ per roo.

Discount
$20 \%$$\left\{\begin{array}{l}\text { Labels. Zinc and Indelible Ink. When indelible ink is used on zinc } 1 \text { abels, a practically } \\ \text { indestructible and always readable label is assured. } \\ \text { Nos. }\end{array}\right.$

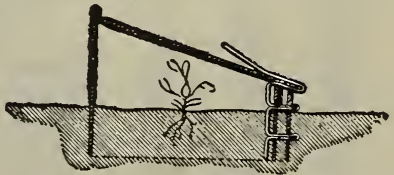



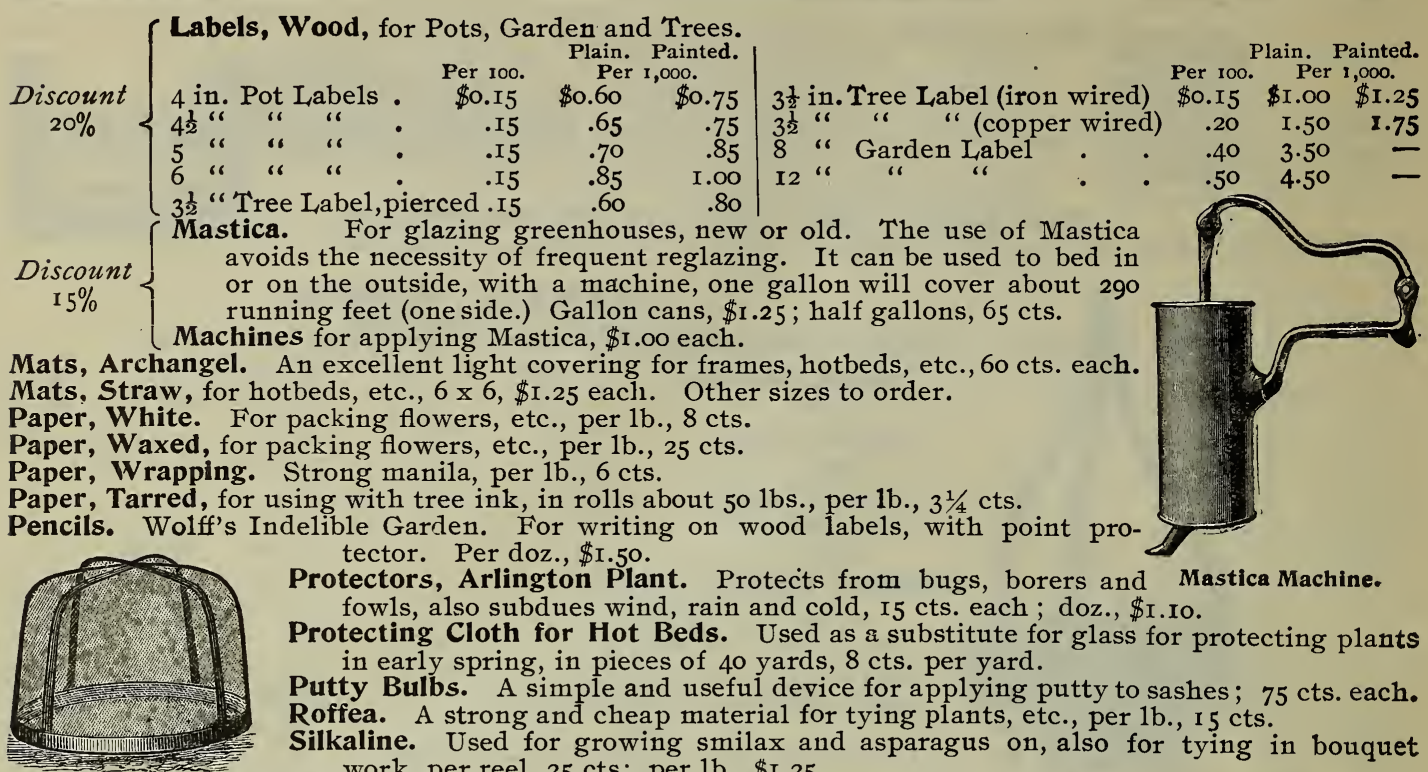
tector. Per doz., \$r.5o.

Protectors, Arlington Plant. Protects from bugs, borers and Mastica Machine. fowls, also subdues wind, rain and cold, I5 cts. each; doz., \$r.Io.

Protecting Cloth for Hot Beds. Used as a substitute for glass for protecting plants in early spring, in pieces of 40 yards, $8 \mathrm{cts}$. per yard.

Putty Bulbs. A simple and useful device for applying putty to sashes; $75 \mathrm{cts}$. each.

Roffea. A strong and cheap material for tying plants, etc., per 1b., 15 cts.

Silkaline. Used for growing smilax and asparagus on, also for tying in bouquet work, per reel, $25 \mathrm{cts}$; per 1b., \$I.25.

Sprinkler, " Ladies' Favorite." This is the most practical sprinkler yet offered to use on house plants, cut flowers or for the laundry. Doz., $\$ 7,50$.

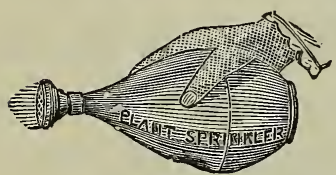

Sprinklers, Scollay's Rubber. An indispensable article for showering plants, sprinkling bouquets, dampening clothes, etc.; three sizes, $\$ 4.50, \$ 6.75$ and $\$ 9.00 \mathrm{doz}$.

Sashes for Hot Beds. Extra well made from thoroughly seasoned lumber, frame, $1 \frac{1}{2}$ in., $3 \times 6 \mathrm{ft}$., $\$ 2.25 ; 13 \%$ in., $3 \times 6 \mathrm{ft}$., $\$ 2.40$ each. Unpainted and unglazer sashes, from $\$$ I. 40 to $\$ 1.60$ each.

Sieves, Wire. For preparing potting soil, sifting loam, gravel, etc., from 16 to 20 inches diameter. $\$ 9.00$ to $\$ 14.00$ per doz.

Stakes, Cane. These are light, strong and durable. They vary in length from 6 to Io $\mathrm{ft}$. Florists use them much, and cut them to any desired length. \$6.50 per Iooo.

Stakes, Unpainted, Hardwood. These are smooth, clean, light, strong and durable, much used for staking carnations, chrysanthemums, etc. $2 \mathrm{ft}$., per 100, $25 \mathrm{cts}$., per 1000, $\$ 1.75 ; 3 \mathrm{ft}$., per 100, $30 \mathrm{cts}$., per I000, $\$ 2.25^{\circ} ; 4 \mathrm{ft}$., per I00, 45 cts., per I000, $\$ 3.25$.

Stakes, Hollyhock. Green painted, extra heavy, $6 \mathrm{ft} . ;$ \$1.00 per doz., $\$ 8.00$ per roo.

Stakes, Galvanized lron Wire. Useful for pot plants and for carnations, etc., in benches. $2 \mathrm{ft} .$, per $1000, \$ 8.50 ; 3 \mathrm{ft}$., per 1000, $\$ 10.00$.

Soil, Prepared Potting, Rotted Fibrous Peat, Leaf Mould. $50 \mathrm{cts}$. per pk., \$1.00 per bu., \$2.00 per bbl. Sphagnum Moss. \$1.00 per bu., \$2.00 per bb1.

Support, Excelsior Wire Carnation. Made in two pieces, no loose rings to annoy, adjustable from 12 to 20 in. in height, per Ioo, $\$ 2.25$; per 1000, $\$ 20.00$.

Supports, Wire Tomato. Very durable, per doz., \$I.25.

Support, "Two Hoop" Wood Tomato. Strong, light, practical, quickly set in position and can be

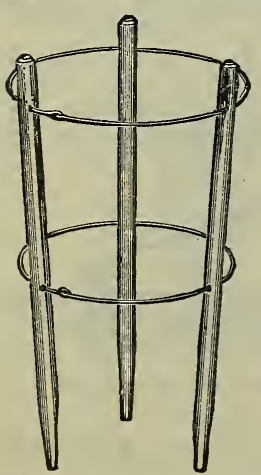
stored from season to season in a small space. Per doz., \$1.co.

Support, "Tie Joint" Tomato. These supports are made of four upright and eight cross pieces of wood that are easily set and retained in position. They are durable, and can be stored away like so many canes when not in use. Per doz, \$r.5o.

Supports, Wood and Wire, Tomato. Uprights of hard wood. Two wire rings. When not in use they fold compactly for storage. Doz., \$I.5O.

Tape, Electric. Exceedingly useful for mending Rubber Hose. It is a strong, adhesive ribbon, three-quarters of an inch wide, can be wound tightly around a leaking part and thus stop it. Per coil, 25 and $50 \mathrm{cts}$.

Tinfoil. For bouquets. Per 1b., I2 cts.

Twine. Soft, for tying cut flowers, vines, etc. Per 1b., $20 \mathrm{cts}$.

Twine, Parceling. All sizes. Per $1 \mathrm{~b}$., Io to $25 \mathrm{cts}$.

Wax, Grafting. (Trowbridge's.) The best in the market. This brand should not be confounded with the many cheap makes now offered, that are not possessed of an even consistency, and give unsatisfactory results. Put up in I $1 \mathrm{~b} ., 1 / 2 \mathrm{lb}$., and $1 / 4$ 1b., pkgs., price, per $1 \mathrm{~b}$., I5, I6 and $17 \mathrm{cts}$.

Wire, Bouquet. Bright, cut in lengths of 9 and 12 inches, in boxes of $12 \mathrm{lbs}$. (one stone) each.

Per box, either length, Nos. 20, 21 and 22 . . \$1.ro| Per box, either length, No. 26 . . . . . \$1.60 " " $"$ " 


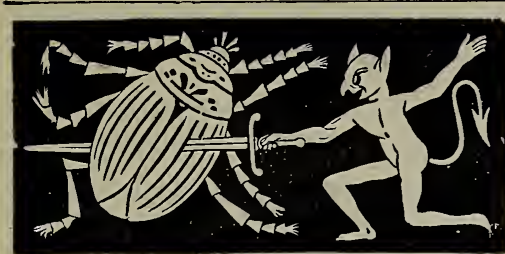

\section{INSECTICIDES ANT FUNGICIDES.}

For Spraying Pumps and Appliances See Hardware Catalogue.

Arsenate of Lead. For spraying trees, etc. Does not burn the foliage. 5 lb. pails, per $1 \mathrm{~b} ., 2 \mathrm{r}$ cents.; Jo $1 \mathrm{~b}$. pails, per $1 \mathrm{~b}$., 20 cts. ; $201 \mathrm{~b}$. pails, per $1 \mathrm{~b}$., I 8 cts. ; $501 \mathrm{~b}$. kegs, per 1b., I 7 cts.

Aphis Punk, Nikoteen. A special preparation of nicotine for the exterminating of green and black fly, thrip, etc., contains no ammonia and does not injure delicate plants. Per box of one dozen rolls (720 grains nicotine) 55 cents.

Bichloride of Mercury. (Corrosive Sublimate.) This article has been found very useful for preventing scab and other fungi disease of potatoes if used on the seed at planting time. We furnish Dr. O. G. Cilley's formula for using, with every package. Per box, sufficient for one barrel potatoes, 25 cts.

Bisulphide. For use in destroying lice on melons, cucumbers and other plants. A light, tight cover of cloth or paper is put over the hill, and under it a teaspoonful of Bisulphide in a small vessel. In an hour every louse under the cover will be dead. Per 1b., $20 \mathrm{cts}$.

Breck's Liquid Anti=Ants. A preparation that destroys or drives away ants from lawns, trees, etc. 1/2 pt., per doz., $\$ 2.50$; pt., per doz., $\$ 4.25$.

Breck's Anti-Ant Powder. A preparation in powder form that contains the same qualities as the preceding, and can be used indoors as effectively as on small plants, flower beds, etc. Two sizes; per doz., $\$ 2.50$ and $\$ 4.25$.

Breck's Dalmation Insect Powder. ("Bubach.”) Manufactured especially for us from clean, dried flowers. It is put up in air-tight packages and keeps its strength indefinitely. It is invaluable to the housekeeper for the destruction of flies, fleas, roaches, and other noxious insects. Packed in four sizes, .6o, \$I. Io, \$I.75, \$3.80 per doz.

Bordeaux Mixture. Powell's. Ready for use by simply adding water. Bordeaux Mixture is, we believe, the best fungicide known, and is really a specific for the blight and other diseases attacking potatoes, tomatoes, grapes, apples, pears, etc. I-1b box, makes 5 gallons spray, I 5 cts.; $5-1$ b box, makes 25 gallons spray, 60 cts.; Io-1b. box, makes 60 gallons spray, \$I.00; 25-1b. box, makes J25 gallons spray, $\$ 2.50$. Discount $15 \%$.

Bordeaux Mixture. French. In pulp form. One gallon makes 50 gallons spray, per gal., \$r.oo; per 5 gals., $\$ 4.50$, per ro gals, $\$ 8.00$. Discount $15 \%$.

Bordeaux Mixture. Lenox Brand. A liquid form. One gallon makes 50 gallons spray, per gal., 75 cts.

Bug Death. A patented non-poisonous powder, entirely different from anything yet offered for the purpose, and as effectual as Paris Green. It is sure death to the potato, squash and cucumber bugs, currant and tomato worms, also other plant and vine eating pests. If used according to directions injury cannot result to the most tender plants, and it has a tendency to assist growth. For potatoes a $12 \frac{1}{2}-1 \mathrm{~b}$. package is sufficient for an acre. I- $1 \mathrm{~b}$. box, I5 cts. ; $3-1 \mathrm{~b}$. box, 35 cts. ; 5-1b. box, 50 cts. ; 121/2-1b. box, $\$$ I.00. Discount $25 \%$.

Bug Exterminator. "The Puritan." For water bugs, roaches and ants. Per doz., $\$ 4.50$.

$$
\begin{aligned}
& \text { Canker Worm Exterminator. (Morrill's Tree Ink.) } \\
& \text { protection to trees from the ravages of grub and } \\
& \text { worms. Directions with each can. }
\end{aligned}
$$

\begin{tabular}{|c|c|c|c|c|c|c|}
\hline $\begin{array}{c}\text { Discount } \\
30 \%\end{array}$ & $\begin{array}{l}\text { 2-1b. Cans } \\
3-1 b \text {. " } \\
5-1 b . \text { " } \\
\text { 10-1b. " }\end{array}$ & & $\begin{array}{l}\dot{\bullet} \\
\dot{\cdot}\end{array}$ & $\begin{array}{l}\dot{\bullet} \\
\dot{\bullet} \\
\dot{\bullet}\end{array}$ & $\begin{array}{r}\$ 0.30 \\
.45 \\
.60 \\
1.10\end{array}$ & $\begin{array}{l}\text { 2o-1b. Cans . } \\
\text { 28-1b. } \\
\text { I 25-1b. Kegs, per 1b. } \\
460-1 b . \text { Barrels, “ }\end{array}$ \\
\hline
\end{tabular}

Black Death. - A new and already very popular insecticide.

Kegs containing Io0 I lb. pkges

Barrels

"6

"

6

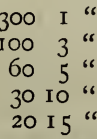

Carbolic Purifying Powder. For removing $\$ 9.00$ per doz.

Carbolic, Crystallized. Per $1 b$. bottle, $\$ 4.00$ per doz.

Carbolic Solution. Per 1b. bottle, $\$ 2.50$ per doz.

Copperdine. Powell's. (A Paste.) A combined fungicide and insecticide, used simply by adding water in proportion of one pound of paste to 16 gallons of water. As a fungicide it prevents black rot, downy mildew and anthracnose of the grape, pear leaf blight, apple scab, mildew, pear cracking. As an insecticide it kills codling moths, curculio, pear insects, peach and apple borers, crown borers, leaf rollers. I-1b. box. makes I6 gallons spray, 25 cts.; 5-1b. box, makes 80 gallons spray, \$I.00; $25-1 \mathrm{~b}$. box, makes 400 gallons spray, $\$ 4.50$. Discount $\mathrm{I} \%$.

Dip, McDougall's Sheep. An effective, non-poisonous preparation for killing vermin on sheep, cattle, and domestic animals. Per 1b., 50 cts.; qt., 75 cts.; 1/2 gal., \$1.50; I gal., \$2.50. Discount $20 \%$.

Dip, Cooper's Sheep. A world-famous preparation, in powder form. It is easily carried, quickly prepared and most effective. Large pkg., sufficient to make 100 gallons, $\$ 1.65 ;$ small pkg., to make 25 gallons, 42 cts. 
Disinfecting Powder, Searle’s. A powerful deodorizer, in powder form. Per doz., \$2.25 For other Disinfectants, see Carbolic, Oil of Tar, Sulpho=Napthol, etc.

Eciffo. A sure cure and prevention of water bugs, cockroaches, etc. Per doz., $\$ 5.00$.

Fir Tree Oil. Soluble. An invaluable preparation for destroying all insects and parasites that infest fruit trees, plants and animals whether on the foliage or at the roots of the plants. Per gal., \$3.25; qt., per doz., $\$ 10.00$; pt., per doz., $\$ 6.50$; $1 / 2$ pt., $\$ 4.50$ per doz.

Fir Tree Uil Soap. This article contains no poison, yet is quite effective in destroying red spider, mealy bug, aphis, etc. It can also be used in the garden against potato bugs, cabbage worms, currant worms, black fly, etc. $1 / 2-1$ b. tin, $\$ 2.25 ; 2-1 b$. tins, $\$ 8.00$ per doz.

Fostite. A light powder that can be easily applied with a bellows; destroys mildew, black rot and rust. Per 5-1b. pkg., 45 cts.; $25-1$ b. box, $\$$ I.70.

Fungiroid or Dry Bordeaux Mixture. In the form of a fine powder applied with bellows or a shaker when the foliage is damp. Said to be as effective as the liquid preparation. I 1b., Io cts.; 5 1bs., 50 cts.

Fungiroid and Paris Green Compound. An insecticide and fungicide combined. Kills potato bugs and prevents blight, easily applied and desirable for fruit trees, etc. I-1b. box, 20 cts.; $3-1 b$ box, 60 cts. Discount $20 \%$.

Grape Dust. Destroys mildew on grape vines. Can also be used in the garden for mould, mildew or rust mites. 5-1b. pkg., $25 \mathrm{cts}$.

Hellebore. Breck's Pure Powdered. We furnish a guaranteed pure article, ground from fresh roots. It is easily applied either in liquid or powder form and destroys currant worms, rose slugs, etc. Each package has special directions for its effective use. Packed in three sizes, air-tight packages, .60, $\$ 1.00$ and $\$ I .80$ per doz.

Kill=M=Right. A soap that is specially prepared to be applied with the "Stott" sprayers. It destroys red spider, mealy bug, aphis, etc. 5-1b. tins, $\$ 1.75$; Io-1b. tins, $\$ 3.25 ; 20-1$ b. tins, $\$ 6$.co. Discount $15 \%$.

Kerosene Emulsion. (A Paste). Ready for use by simply adding water. This is strictly an insecticide for plant lice of all kinds, rose bugs, cabbage bugs, melon insects, fowl, human and animal lice, caterpillars, tobacco flies, peach lice, red spider, green fly and all sucking insects. I-1b. can makes ro gallons spray, I5 cts.; 5-1b. can makes 50 gallons spray, 6o cts.; 25-1b. can makes 250 gallons spray, $\$ 2.50$; $300-1 \mathrm{~b}$. bb1. makes 3.000 gallons spray, $\$ 24.00$. Discount $15 \%$.

Little's Antipe t. A safe and sure preparation for the destruction of all insect pests, equally suitable for greenhouse, garden and orchard. Per pt., $40 \mathrm{cts}$.; qt., 8o cts.; gal, $\$ 2.00$.

London Purple. For spraying fruit trees, potatoes, etc. I 1b., I6 cts.

Lemori Oil. An oil that is readily soluble in water. It destroys insects and parasites of all kinds without injury to plants. Much used for palms and other ornamental foliage plants. Pt., 35 cts.; qt., 65 cts.; gal., \$2.co.

Nicomite. A new fumigating compound in powder form. It is used for destroying green and black fly, thrip, mealy bug, etc., in greenhouses and frames. It may be used in houses containing tlie most tender plants in full bloom without injury resulting, and its use does not affect the natural scent of the flowers. No. I tin, for I6,000 cubic feet of space, $\$ 3.00 ;$ No. 2 , for 7,500 feet, $\$ 1.50 ;$ No. 3 , for 5.000 feet, $\$$ I.00; No. 5 , for $I, 750$ feet, 50 cts. Fumigators with lamp for applying made to contain sufficient for 5,000 feet, $50 \mathrm{cts}$. each. Discount $15 \%$.

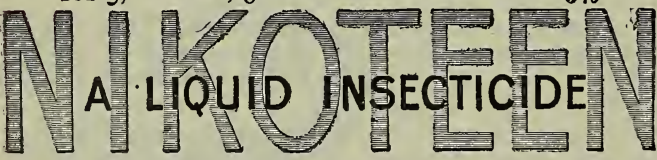

Nikoteen. Packed in pint bottles; each bottle contains all the Nikoteen obtained from two hundred pounds tobacco stems. A cheap, clean and $\cdot$ safe insecticide. Pt. bottle, \$I.30.

Oil of Tar. Prepared from coal tar, and retains the carbolic acid of the tar in a liarmless form. It mixes with water in any proportion. It is a disinfectant perfect in action, agreeable in odor, non-poisonous, cheap and unsurpassed for use in the home, the stable, kennel, or wherever a.disinfectant is required. Circulars detailing its many uses mailed free upon application. Qt., $25 \mathrm{cts}$; 1/2 gal., $50 \mathrm{cts}$.; gal., \$I.00. Discount $25 \%$.

Paris Green and Fungiroid. Per 1b., 20 cts.; 3-1b. box, 60 cts. Discount $20 \%$.

Paris Green, Breck's Guaranteed Pure. 1/4 1b., I9 cts.; 1/2 1b., I 8 cts.; I 1b., I 7 cts. per $1 \mathrm{~b}$.

Quassaine. (A Paste.) Ready for use by adding water. This article is a non-poisonous insecticide, a specific for San Jose scale, red spider, aphis, hop lice, borers and scale insects of all kinds. I-1b. can makes 25 gallons spray, 25 cts.; 5-1b. can makes 125 gallons spray, \$1.25. Discount ${ }_{1} 5 \%$.

Slug Shot. Not only acts as an insect destroyer, but it will be found to be an excellent fertilizer. It contains nothing injurious to domestic animals, and does not deteriorate by age. On low-growing plants it should be dusted on with a very fine sifter. On tall shrubs and trees it should be applied with bellows. Price, 5-1b. pkg., 20 cts.; ro-1b. pkg., 40 cts.; in barrels of about $2251 \mathrm{bs}$., per $1 \mathrm{~b}$., $3 \frac{3}{4} \mathrm{cts}$.

Soa, Pinner's Tobacco. Manufactured from the essential oil of tobacco, for the destruction of all insects, parasites and their eggs. Per 1b., 30 cts.

Soap, Whale Oil. For destroying insects on plants, trees, vines, etc., for washing down the bark of trees, grapevines, etc. In boxes, I-1b., $\$ \mathrm{I} .20 ; 2 \mathrm{I} / 2-1 \mathrm{~b} ., \$ 2.25$ per doz.

Soap, Sulpho=Tobacco. Rose's Perfected. Does perfect work on all insects affecting flowers, trees and animals. In tin boxes, sufficient for Io gals. of water, $\$ 2.25$ per doz.

Soap, Permol=Kerosene. This soap contains $25 \%$ of kerosene oil, and although solid in all ordinary temperatures it is quickly soluble in any desired proportion of water. It combines the properties of a "Resin Wash" and "Kerosene Emulsion." It destroys scale of every kind, aphides or active plant lice and all sucking insects. I 1b. makes from 16 to 40 gallons wash or spray, according to the enemies to be overcome. Full directions for its use accompany each package. I-1b. pkg., 20 cts.; 25-1b. pkg., I4 cts. per 1b.; 50-1b. tubs, 12 cts. per $1 b$. 
Soap, Permol=Copper. This preparation contains copper in a form practically soluble in water, until thoroughly dried. In using it for mildew, rust, smut, and such fungi a strength of $11 \mathrm{~b}$. to 30 gals. of water is recommended. Do not dissolve it in iron or zinc utensils. I-1b. tins, 65 cts; ro-1b. tins, 60 cts. per $1 \mathrm{~b}$.

Spanish Pink. A safe and effective article for the destruction of cabbage and web-worms, caterpillars, and other chewing insects that affect cabbage, squash, cucumbers, melons, etc. Non-poisonous, 25 cts. per $1 b$.

Sulphate of Copper. (Blue Stone). Per 1b., $7 \frac{1}{2} \mathrm{cts}$.

Sulphur, Flowers of. Used to prevent and cure mildew on plants. $\$ 4.25$ per I $001 \mathrm{bs}$.

Tobacco Extract. Black Squali Brand. Pure, highly concentrated; hydrometer test, after Beaume's scale 40 degrees, temperature 60 degrees. Fahrenheit, being of uniform strength, is always effective. It can be applied by vaporizing or spraying. It effectually destroys green fly, red spider and scale. Per pt., $\$ 2.00$ per doz.; per qt., $\$ 3.50$ per doz.; per gal., 80 cts. ; 5 gals., $\$ 3.25$.

Tobacco Extract. Rose Leaf Brand. A perfectly pure, aqueous extract, highly concentrated and always uniform in strength of nicotine poison. Destroys green fly, mealy bug, red spider, and scale. Pts., $\$ 2.25$ per doz; qts., $\$ 4.00$ per doz.; I gal.; $\$$ r.oo; 5 gals., $\$ 4.25$.

Tobacco and Sulphur Powder. Prevents and cures mildew. Kills green and black fly and other insects. $\$ 5.00$ per $1001 \mathrm{bs}$.

Tobacco Dust. Very finely pulverized. A valuable insecticide and fertilizer. \$2.50 per 1001 bs.

Tobacco Stems. For fumigating. If used as a mulch around sweet peas and other plants they prevent the destructive work of the wire and cut worms. $\$ 1.50$ per bale.

$\mathbf{X L}=\mathbf{A l l}$ Liquid Insecticide. For syringing, spraying, dipping, or sponging. Destroys all insects, especially effective against mealy bug and red spider. Can be used without injury in graperies, even when the fruit is in an advanced stage of growth. Pt., 65 cts.; qt., $\$ 1.00$. Discount $20 \%$.

$\mathbf{X L}=\mathbf{A l l}$ Vaporizing Compound. This is an English preparation, that at the first glance has a highpriced appearance. The safety with which it can be used, its never-failing effectiveness and the ease with which it can be applied as an insecticide, places it at the head of the first rank. It is cheaper, results considered, than any other vaporizing compound, and there is no other like it. Its action is positive on Mealy Bug, Thrip, and all kinds of Aphis. Price per bottle No. I, \$8.25; No. 2, \$4.50; No. $3, \$ 2.25$; No. 4 , $\$$ I.I5. The smallest bottle is sufficient to fumigate 5,000 cubic feet. Discount $20 \%$.

Fumigators. With lamp for vaporizing: large, $\$ \mathrm{I} .00$; medium, 85 cents. Discount $20 \%$.

\section{TOUGH ON FLIES.}

This is the original preparation for the purpose of preventing insects attacking horses, cattle, anu other domestic animals. We have sold it for ten years; each year with largely increased sales. We confidently recommend it. as experience has proved it to be as represented, in every particular. Harmless and effective. One application

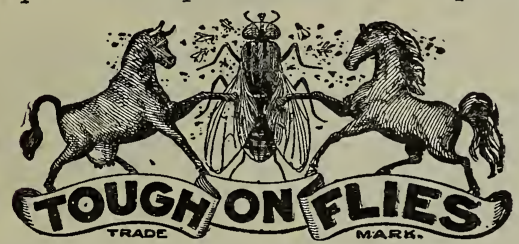
instantly and positively relieves horses and cattle from all annoyance from Green Heads, the dreaded Texas Heel Fly as well as other flies, gnats and insects of every description for at least twenty-four hours. It improves the coat of the animal and abolishes the use of fly nets. It prevents thrush and other hoof diseases. It is soothing and healing if applied to sores, and stands unrivalled as a disinfectant. Applied to cows, its wonderful effect in securing for them perfect rest and a chance to feed in peace quickly manifests itself in their improved appearance and by the increased quantity of milk given. It is the greatest compound of this progressive age. Recominended by thousands using it. One gallon lasts four head of horses or cattle an entire season. Price of "Tough on Flies": Qt. cans, $\$ 4.50$ per doz.; $1 / 2$ gal. cans, $\$ 9.00$ per doz.; I gal. cans, $\$ 15.00$ per doz.; 5 gal. cans, $\$$ I. I 5 per gal.

\section{ANTI-FLY.}

A harmless, but effective preparation of drugs and chemicals to relieve horses and cattle from the annoyance of flies and other insects. It contains no tar, nor does it spoil the appearance of the aninal's coat. Price, per qt., $\$ 4.50$ per doz.; $1 / 2$ gal., $\$ 9.00$ per doz. ; per gal., $\$ 15.00$ per doz. ; 5 gal. cans, per gal., $\$ 1$.I 5

\section{INSECTICIDE DISTRIBUTORS.}

For spraying apparatus, pumps, etc., see our large Implement Catalogue, 360 pages, free to customers.

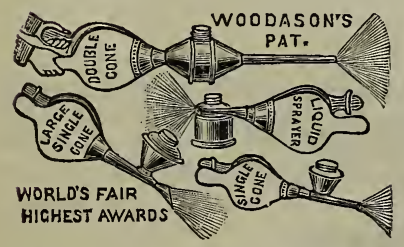

Bellows. Woodason's for liquid and powder. These are really high-grade goods that will, with proper care, last many years and do satisfactory work.

Small Single Cone for applying powder, doz. . . . . . \$ \$ 2.00

Large " " " " " . . . 24.00

Double Cone " " " "

Sulphur Bellows, doz. .

Large, Liquid Spraying, doz.

Medium, Liquid Spraying, doz. .

Small

17.00

22.00

17.00

Bellows, Houchin's Insect Powder. Strongly made of good material. These will be found very serviceable for applying insecticides in powder form.

No. 9, holds 2 oz. powder, $\$ 4.50$

" I I, " 3 " 3 "

Doz

o. 16, holds $6 \mathrm{oz}$. powder, $\$ 7.00$

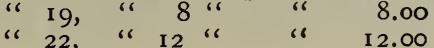

I 2.00 
Dusters, Tin. These have finely perforated bottoms for applying Paris Green, Slug Shot, etc. $\$$ I.5० per doz.

Gun, Excelsior Powder. For applying insect powders, etc. $\$ 1.00$ per doz.

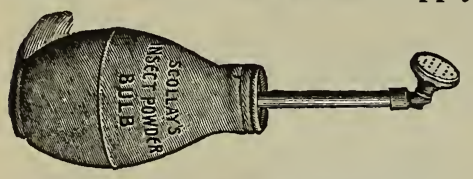

Cyclone Powder. $\$$ r.0o per doz.

Blizzard Powder. \$o.65 per doz.

Powder Puff, Scollay's Insect. This is a large, strong rubber bulb, with long metal neck and distributer set at an angle that enables the operator to apply either powder or liquid over or under the leaves. \$9.00 per doz.

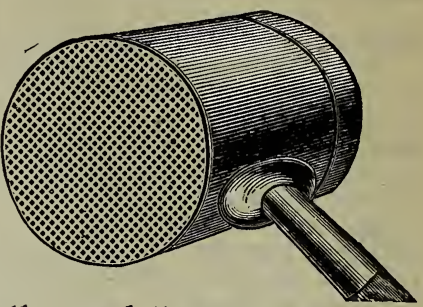

Shaker Perfection Powder. This is well named "Perfection," for it is the most perfect and easiest to use of any shaker yet offered. It was gotten up especially to apply Bug Death, but it will be found useful for applying Plaster Dust, Paris Green and Plaster or any other powder. The bottom is made of copper and finely perforated. The powder is distributed by a swinging, semi-circular motion. Doz., $\$ 7.80$. Discount $25 \%$.

\section{FER'TILIZERS.}

\section{BRECK'S LAWN AND GARDEN DRESSING.}

The verdict of all who have used Breck's Lawn and Garden Dressing is that it is the most complete and satisfactory Lawn Dressing ever placed upon the market. It is manufactured expressly for us and we vouch for its containing all the constituents required to produce a luxuriant growth. We believe beyond a question that ours is the only absolutely odorless Lawn Dressing. It is also so clean that the most fastidious person can apply it without offending the organs of sight and smell. It being quick in action, the effect can be seen immediately after the first rain or the sprinkling of the hose, and going directly to the roots of the grass, stimulates them to greater activity, thereby thickening the sward and producing a firmer growth, which remains rich, green and velvety throughout the season. Its superiority over stable manure can scarcely be estimated, as the latter, in addition to disfiguring the lawn by its unsightly appearance, also gives out an unpleasant odor, and always

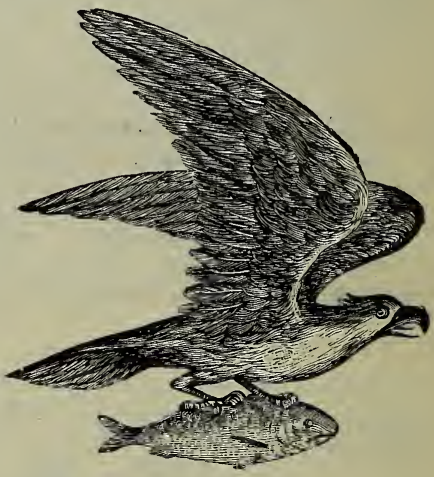
contains weeds and other undesirable seeds. Great care should be taken to distribute the dressing evenly, and in no greater quantities than recommended on the tag which accompanies each bag, as a too liberal application will injure the sward. It can be applied at any time during the year, from A pril to November, as often as the grass seems to need nourishment. In making new lawis apply at the same time the seed is sown and rake in with the seed. Never apply the dressing while the grass is wet with dew or rain. Any time the grass is perfectly dry will answer, but the best time to apply it is just before a shower. It is equally as suitable for flower beds and all vegetable crops as for the lawn.

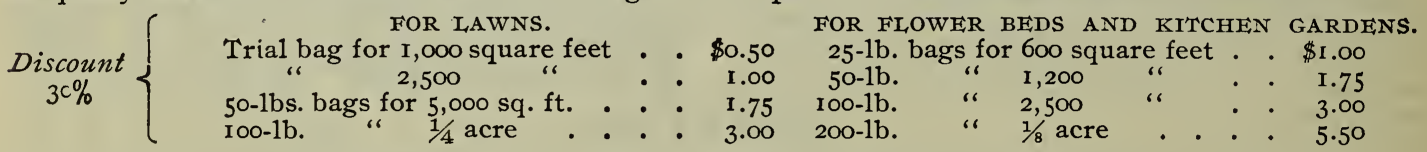

\section{BRECK'S MARKET GARDEN MANURE.}

A high grade, properly balanced, chemical fertilizer that is expressly recommended to market gardeners and farmers for potatoes and all vegetable crops. We sold large quantities of it last year, and already we have had many testmonials, also increased orders from those who had it for use Spring of 1899 . It is beyond a question the best value offered in Chemical Fertilizers. 5o 1bs., per ton, $\$ 32.00$; $1001 \mathrm{bs} .$, per ton, $\$ 30.00$.

\section{PERUVIAN GUANO.}

For a number of years there has been some difficulty in obtaining a desirable grade of this article. When a good quality is to be had there is much satisfaction resulting from its use, especially on early vegetables and quick growing crops. We have made arrangements with the importer of the best brand that sells so freely in Britain and offer our patrons the genuine "Peruvian" in original bags.

Per ton, $\$ 45.00$.

\section{UNLEACHED MARD WOOD ASHES.}

Our trade in this valuable fertilizer has grown to such an extent that we have for a number of years had an experienced agent employed in Canada collecting the purest, driest and choicest grade of Hard Wood Ashes that is obtainable. We guarantee the percentage of potash and to insure against too much water we accept none that contain more than I2 per cent moisture. We quote special prices on carloads in bulk delivered anywhere. Price per ton, in barrels, f. o. b. Boston, $\$ 13.00$; per barrel, f. o. b. Boston, $\$ 1.65$. 


\section{SHEEP MANURE.}

This is a pure natural manure, and nutritious food for plants. Its effect is immediate. It is the best of all manures for mixing with the soil for greenhouse plants - one part manure and six parts soil. Price, in bags of 100 lbs. each, $\$ 1.75$; per ton, $\$ 30.00$.

\section{JADOO FIBRE AND LIQUID.}

A chemically prepared vegetable fibre, rich in plant food, and from its peculiar character is especially suitable to induce root growth and cause healthy, vigorous plants. For sprouting seeds there is nothing equal to it.

Fibre. Per ton, $\$ 30.00 ;$ I 001 bs., $\$ 1.75$.

Liquid. Ten gal. can, $\$ 6.00 ; 5$ gal., $\$ 3.25$.

Jadoo preparations

net f. o. b. Boston.

\section{IMPERIAL LIQUID FERTILIZER.}

This new departure in fertilizers bids fair to become popular as soon as its merits are known. Being in liquid form and immediately soluble, it will be found of great value to those who grow vegetables or flowering plants under glass. Last season it was extensively used on Putting Greens with pleasing results. $\left.\begin{array}{l}\text { Imperial Liquid Vegetable Fertilizer } \\ \text { Grass Fertilizer. }\end{array}\right\}$ Before using dilute with 50 times its bulk of water.

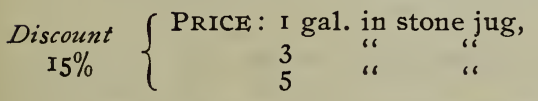
$\$ 0.85$ Containers Included.
2.15
3.30
f. o. b. Boston.
$\left\{\begin{array}{lr}\text { PRICE : Io gal. cask, } & \$ 5.25 \\ 25 & 10.50\end{array}\right.$ $3 \cdot 30$ )

\section{SCOTCY SOO'T.}

This is much used by florists and gardeners, both as a fertilizer and fungicide. Mixed with Flowers of Sulphur (two-thirds soot, one-third sulphur), there is nothing better to prevent and cure mildew. Used as a fertilizer, either in the dry state or in liquid, it quickly promotes growth and heightens the color of both flower and foliage. Per roo-1b. bag, \$2.0o.

\section{CHEMICALS.}

Net f. o. b. Boston. Prices subject to change.

Dried Blood, 12 to 14 per cent ammonia,

"

Fine Ground Tankage, 9 to ro per cent ammonia, 15 to 20 per cent bone phosphate,

Sulphate of Ammonia, 24.75 to 25.25 per cent ammonia,

Nitrate of Soda, 94 to 96 per cent purity, i 8 to 20 per cent nitrogen, estimated as ammonia,

Dry Ground Fish, 9 to I I per cent ammonia, i 5 to 20 per cent bone phosphate

Dissolved Bone, 2 to 3 per cent ammonia, i 2 to 14 fer cent phosphoric acid

Dissolved Bone Black, I5 to 18 per cent phosphoric acid

Plain Superphosphate, I2 to I 5 per cent phosphoric acid

Ground S. C. Phosphate, 57 to 62 per cent bone phosphate

Muriate of Potash, 80 to 85 per cent muriate of potash, 50 to 55 per cent actual potash

High Grade Sulphate of Potash, 90 to 95 per cent sulphate of potash, 50 to 52 per cent actual potash

Double Sulphate of Potash and Magnesia, 48 to 52 per cent sulphate of potash, 35 to 40 per cent sulphate of magnesia, and not over $2.5^{\circ}$ per cent chlorine, 26 to 28 per cent actual potash.

Sylvinit

Kanit, 23 to 25 per cent sulphate and muriate of potash

Sulphate of Magnesia (Keiserite), 50 to 55 per cent sulphate of Magnesia

Sulphuric Acid, 100 to I 30 lbs. in carboy. (Carboys extra, $\$ 1.50$ each)

Land Plaster, Fine Ground, $2001 \mathrm{~b}$. bags,

Piaster Dust, 5 lb. box, I 5 cts.; 25 lb. bag, 50 cts.; barrels of about 150 1bs., $\$ 2.00$ net.
Perton. $\$ 37.50$ 33.00

35.00

28.00

70.00

44.00

32.00

27.00

20.00

I 6.00

I 6.00

43.00

48.00

28.00

I 5.00

I 3.50

14.00

35.00

7.25

8.00

\section{FINE GROUND FERTILIZER BONE.}

We have a high grade of this valuable fertilizer specially prepared for us. It is useful for all crops but especially for grass lands and fruit trees. Per 50-1b. bag, $\$ 30.00$ per ton; Ioo-1bs., $\$ 28.00$ per ton F. U. B. BosToN.

If you have not received a copy of the SECOND EDITION of our CATALOGUE OF AGRICUL= TURAL HARDWARE, IMPLEMENTS, MACHINES AND WOODENWARE, please advise us so that one may be sent you. It contains 360 pages, over 1,500 illustrations and is of great value to every dealer. 


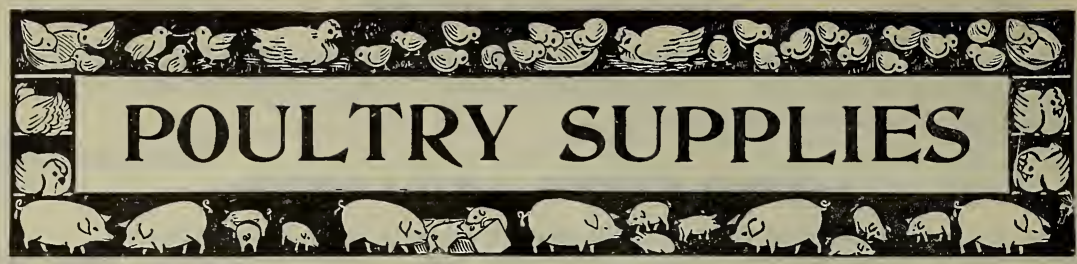

(Prices subject to change.)

\section{BRECK'S POULTRY AND SWINE MEAL.}

This article is the most complete animal food for poultry obtainable. It is concentrated, pure, palatable and effective. It was first offered to the American public in 1892 , but it has since that time found its way into the yard of the most progressive poultry men in the country, because it is superior in every respect to all other meals made from meat or animal matter.

roo $1 \mathrm{~b}$. bag, $\$ 35.00$ per ton; $50 \mathrm{lb}$. bag, $\$ 36.00$ per ton; trial bag (a month's supply for thirty hens $\$ 40.00$ per ton.

Ground Oyster Shells. Ioo 1b. bags, per ton, $\$ 7.00$.

Mica Crystal Grit. Ioo 1b. bags, per ton, $\$ 9.00$.

Cracked Poultry Bone. Per bbl. of about 200 lbs., per ton, $\$ 38.00$.

Bone Meal for Poultry and Cattle. Per ton, $\$ 38.00$.

Flour of Bone. Per ton, $\$ 40.00$.

Ground Beef Scraps. roo 1b. bags, per ton, $\$ 39.00$.

Fish Meal. (Desiccated Fish.) roo 1b. bag, per on, \$35.00.

Wheat. $\$ 28.00$ to 35.00 per ton, according to quality.

Wheat Screenings. $\$ 22.00$ to $\$ 28.00$ per ton, according to quality.

Green's Poultry Food. A mixture of granulated cereals, grand as a scratching food for both chicks and adult fowls. Ioo $1 \mathrm{~b}$. bags, $\$ 35.00$ per ton.

Spratt's Food. The celebrated English preparation, nothing equals it for young chicks. $50 \mathrm{lb}$. bag, $\$ 3.00$.; 1001 bs., $\$ 5.50$. Discount 10\%.

Barley. $6_{5} \mathrm{cts}$. per bu.

Buckwheat. 90 cts. per bu.

Peas. Small, white Canada, $\$$ i.25 per bu.

Sunflower. Large Russian, 4 cts. per $1 b$.

Millet. Golden, for chickens, $\$ \mathrm{I} .20$ per bu. of $501 \mathrm{bs}$.

Hemp, Large Russian. Much fed to pigeons and fowls that are being fattened for the table, $\$ \mathbf{I} .40$ per bu. of $401 \mathrm{bs}$.

Cut Clover. $501 \mathrm{~b}$. bags, $\$ 35.00$ per ton.

Clover Meal. $501 \mathrm{~b}$. bags, $\$ 35.00$ per ton.

Kaffir Corn, or White Sorghum. A favorite food with pigeon fanciers, and since we have offered it at a low price poultry men have fed it with best results. Per I oo 1 b. bag, $\$ \mathbf{1} .25$.

Breck's Eureka Egg Food. This preparation stands without a rival, as hundreds of those who have used it testify. It aids digestion, prevents and cures many diseases common to fowls, and although the best food known to produce eggs, it does not desiroy the fertility of the egg. I $1 \mathrm{~b}$. box, $\$ 2.00$ doz.; $2 \frac{1}{2} 1 \mathrm{~b}$. box, $\$ 4.00 \mathrm{doz}$; 6 1b. box, $\$$ r.00 each; Io 1b. box, $\$ 8.00$ doz. ; $251 \mathrm{lb}$. kegs, $\$ 2.50$ each.
Sheridan's Condition Powders. $\$ 2.25$ and $\$ 9.00$ per doz.

Rust's Egg Producer. I 1b. box, $\$ 3.00$ per doz.; $2 \frac{1}{2} 1 \mathrm{~b}$. box, $\$ 6.00$ per doz. ; $61 \mathrm{~b}$. box, $\$ \mathrm{I} 2.00$ per doz. ; Io 1 b. box, $\$ 18.00$ per doz.; 25 lb. kegs, $\$ 42.00$ per doz. Discount $30 \%$.

Rust's Haven's Roup Pills. These pills act directly on the glands and mucous membrane, allaying all tendencies to fever and carrying off ali morbid matter from the system. Box of 48 pills, $\$ 3.00$ per doz. Discount $30 \%$.

Rust's Haven's Climax Condition Powder. Small package, $\$ 3.00$ per doz. ; $21 \mathrm{~b}$. box, $\$ 6.00$ per doz.; 5 lb. box, $\$ 12.00$ per doz. Discount $30 \%$.

F. P. C. Chick Manna. I 1b. Io cts.; 5 1bs., 40 cts.; 15 lbs., $\$ 1$.1o; 60 lbs., $\$ 4.20$. Discount $15 \%$.

Madoc Gape Cure. Small, 20 cts. ; large, 35 cts. ; Discount $\mathrm{I} 5 \%$.

Multum in Parvo Poultry Powder. Sma11, 20 cts. ; large, 35 cts. Discount $15 \%$.

Charcoal, Granulated. Per 100 lbs., $\$ 5.00$.

Charcoal, Powdered. Per I 001 bs., $\$ 4.00$.

Chalk, Powdered. 2 cts. per $1 \mathrm{~b}$.

Copperas. (Green Stone.) 2 cts. per $1 \mathrm{~b}$.

Carbolic, Crystallized. I 1b. bottles, $\$ 4.00$ per doz.

Carbolic Acid Solution. I 1b. bottles, \$2.50 per doz.

Carbolic Purifying Powder. Small, $\$ 2.25$ per doz. ; large, $\$ 9.00$ per doz.

Dalmation Insect Powder. Per 1b., 30 cts.

Slug Shot. This article is not only a valuable insecticide but also a powerful disinfectant. Per $5 \mathrm{lb}$. pkg., 20 cts.

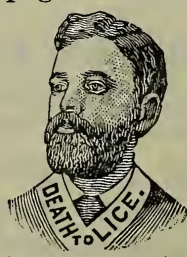

Death to Lice. I 5 oz. box, \$I.50 per doz.; 3 1h. pkg., $\$ 3.60$ per doz.; I 00 oz. pkg., $\$ 7.20$ per doz.

Death to Lice, Special. A preparation containing all the qualities of the famous "Death to Lice Powder," but which is soluble in kerosene oil and can be used to paint or spray roosts and interiors of poultry houses. I lb. box, $\$ 1.5^{\circ}$ per doz.

Death to Lice Ointment. For use on young chicks. $60 \mathrm{cts}$. and $\$ 1.50$ per doz. 
POULTRY SUPPLIES.-Continued.

“ O. E. C." Lice Powder. An excellent article. It not only is death to all insects but acts as a powerful yet pleasant disinfectant. Per doz., $\$ 4.00$.

P. D Q. Powder. A valuable preparation that not only destroys and prevents insects of all kinds on fowls, but drives away quickly all sorts of insects that pester the housewife. It is useful for a hundred and one purposes and not the least among them is that of disinfectant. Per doz., $\$ 2.00$ and $\$ 7.80$.

Rice's Lice Paint. For poultry and all domestic animals. Kills ticks, mites, lice, fleas and bed-bugs. Easy to apply. 2 qt. can, per doz., $\$ 4.50$.; I gal. can, per doz., $\$ 6.75$

Tobacco Dust. An exceedingly fine powder. Valuable as an insecticide. Per Ioo lbs., \$2.50.

Tobacco Stems. Used to mix with nest material, fumigating, etc. Per bale, $\$$ I.5o.

Sulpho=Napthol. This preparation, originated by the late Dr. Cabot, is recommended for a great many purposes. It cleans and heals all sorts of cuts, bruises and sores. It is sure death to all kinds of bacteria and insects and is unsurpassed as a disinfectant. For domestic animals, poultry and poultry houses it is the thing. Per doz., $\$ 4.00$ and $\$ 8.00$.

Oil of Tar. A new coal tar preparation, somewhat similar to Sulpho-Napthol. Per qt., 25 cts. ; $1 / 2$ gal., 50 cts.; I gal., $\$$ 1.00. Discount, $25 \%$.

Veterinary $\mathbf{X}=$ Zalia. A purely vegetable preparation, contains no alcohol or poisonous ingredients. It may be applied to a raw surface or even poured into the eye of an animal without causing pain. It cures Galls, Scratches, and Surface Inflammation of every description. Per doz., \$Io.oo.

Plaster. Land. A valuable absorbent, when mixed with the droppings it deodorizes, also enhances their value as a fertilizer. I00-1b. bags, $\$ 8 . \infty$ per ton.

Nest Eggs, China. Per gross, $\$ 2.75$

Medicol Nest Eggs. These are medicated eggs but entirely different from anything that has hitherto been offered. They can be cleaned without injury, are lasting, effective, and appreciated by chickens and adult fowls. No hen should be set without one of these in the nest as a prerentative of vermin. One placed in a bird cage keeps it sweet and the bird free from insects. They drive away lice from pot plants, prevent moths from attacking furs or other clothes when packed away, and are also valuable as a disinfectant. Per gross, $\$ 9.00$

Bellows, Spraying. Per doz., $\$ 12.00$ to $\$ 22.00$.

Bellows, Houchin's Patent Powder. Per doz., $\$ 4.50$ to $\$ 12.00$.

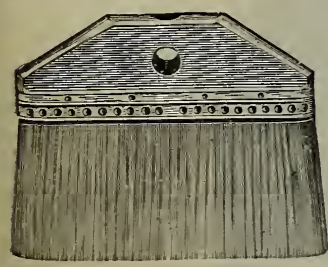

Brushes, Whitewash. Tampico centre, bristles outside. Per doz., $\$ 3.35$, $\$ 3.75$ and $\$ 4.25$.

Nests, Wire. Strong and clean. Per doz., \$3.50.

Leg Bands. The famous Phœnix, secure, neat, light and superior to all others. Numbered to order. Per IOO, \$1.25.

Leg Bands, Aiuminum, for Pigeons. Seamless, per doz., 30 cts. ; per I00, $\$ 2.00$. Open, per doz., 20 cts.; per 100, \$1.00.

Leg Bands, Aluminum, for Poultry. Per doz., 20 cts. ; per I0o, \$1.00.

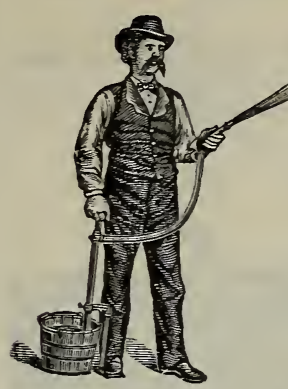

Pumps, Breck's Hand Force is a double-acting portable force pump. It is made of brass, and therefore impervious to the action of acids and liquids, and will not rust or corrode. Pump is placed directly in water or other liquid, insuring a perfect suction. It is furnished complete with malleable foot-rest and suitable nozzles for a solid stream and large spray. Pump may be used for washing windows, wagons, in conservatories, gardens, etc., and for general spraying purposes. Price, $\$ 3.75$ each.

Guns, Insect Powder. 2 oz., \$1.00 doz.

\section{STONE DRINKING FOUNTAIN.}

This fountain is of the best patent stoneware, and is very desirable.

$1 / 2$ gallon each $\quad$ - . . Per doz., $\$ 2.50$

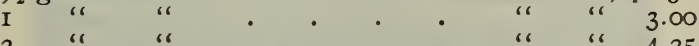

“E. H. M." Stone Fountains. Made of glazed stoneware, in two pieces; a saucer and an inverted cup, finished off on top to prevent roosting. Strong, cheap, easily cleaned and quickly filled. The best stoneware fountain yet offered. The one quart size is especially suitable for pigeons. I qt., per doz., $\$ 2.00 ; 2$ qts., $\$ 2.75$; I gal., per doz., $\$ 3.25$.

Sanitary Fountain. Square in form, made of galvanized iron, in two pieces, easily cleaned and filled. I gal., per doz., $\$ 5 \cdot 50 ; 2$ gal., per doz., $\$ 8.50$.

\section{HUB DRINKING FOUNTAIN.}

This fountain is made of the best galvanized iron, is very strong, easily cleaned, quickly filled, and is equally serviceable for both chickens and adult fowls (see cut). This fountain is vastly superior to all others. I gallon .

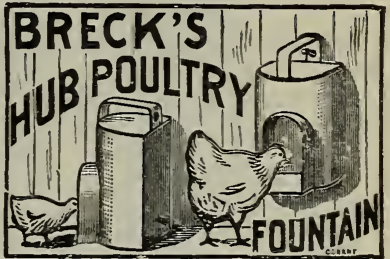

Per doz, $\$ 6.00$

2 " three ply Tarred Roofing, Ruberoid Roofing, Red Rope Roofing Paper, etc. Price upon application.

Caponizing Instruments. We carry a full line of the best makes and approred patterns. Prices, from $\$ 2.50$ to $\$ 7.00$ per set. Discount, $10 \%$.

Knives, French Poultry Killing.

When this knife is properly used there is no complaint as to imperfect bleeding of broilers or roasters. Per doz., $\$ 4.8$ o.

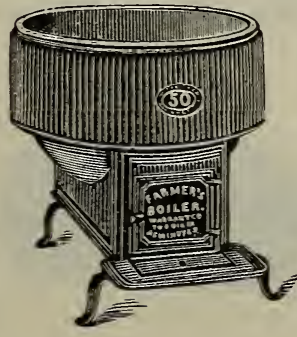

FARMER'S BOILERS.

\begin{tabular}{|c|c|c|c|}
\hline Cap & $\begin{array}{l}\text { city. } \\
\text { a!s. }\end{array}$ & & $\begin{array}{l}\text { Mfrs. } \\
\text { Price. }\end{array}$ \\
\hline I5, & for & wood, & $\$ 15.00$ \\
\hline 20 , & " & 6 & 16.00 \\
\hline 25 , & " & " & 20.00 \\
\hline 30, & " & "6 & 24.00 \\
\hline 40 , & "6 & $" 6$ & 30.00 \\
\hline 50 , & "6 & " & 40.00 \\
\hline 65 & " & " & 49.00 \\
\hline 30 , & “" & coal, & 30.00 \\
\hline & 6 & & 40.00 \\
\hline
\end{tabular}

Disc. $35 \%$. 
POULTRY SUPPLIES. - Continued.

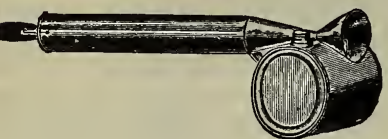

Sprayer, Cyclone. Tin, per doz., \$4.75 Brass, “ 10.50

Baskets for Ship= ping Eggs. These are especially arranged for the safe shipment of eggs for hatching, and are plainly marked, "Eggs for Hatching. Handle with Care." Price, complete with boxes, one setting size, per doz., \$I.50; two set-

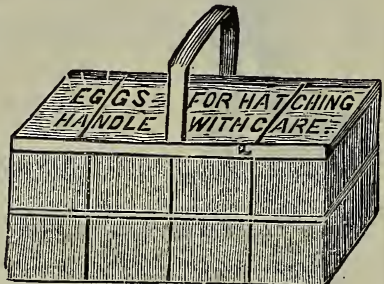
ting size, \$2.00. Sample basket complete, 25 cts. Net.

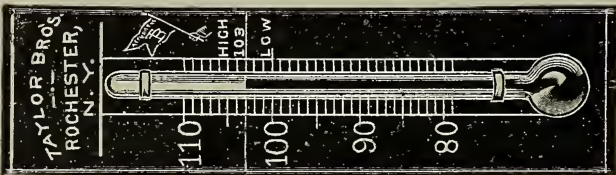

THERMOMETERS. 3.00

For Brooders. . Tested and warranted. Per doz.,

Prairie State Upright. Per doz., $\$ 5.00$

Household Standard. 8 in., per doz., \$6.50; ro in., per doz., \$8.00; I 2 in., per doz., \$9.50.

Dairy Floating. Per doz, \$1.50.

Sixe's Self Registering. Per doz., \$27.00.

Cookers, Granite State Feed. Thoroughly adapted for the purpose.

Cookers, Empire State Feed. 25 gal. size, $\$ \mathrm{r} 0.00$; Io gal. size, \$I 5.00. Discount 10\%.

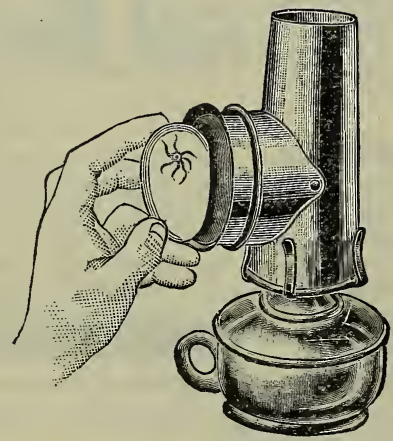

Egg Tester. Will fit any lamp with a No. 2 burner. Per doz., \$3.00.

Rat and Mouse Traps. French, all wire, 90 cts. each. Square, all wire, 40 cts. each. Erie, 20 cts. each. Mouse Traps from 5 cts. to $40 \mathrm{cts}$. each.

Discount $20 \%$.
MANN'S BONE CUTTER.

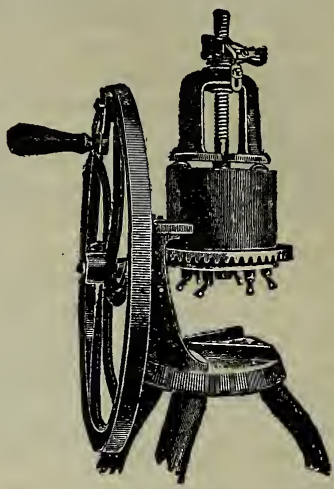

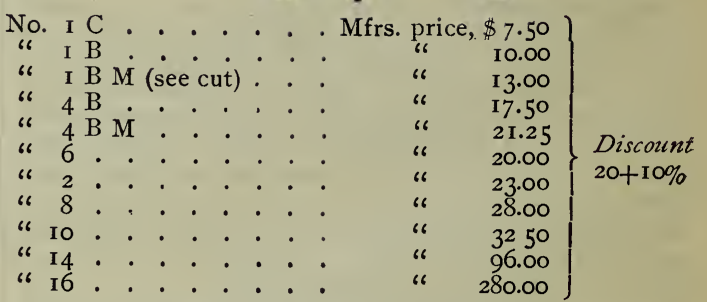

MANN'S FEED TRAYS.

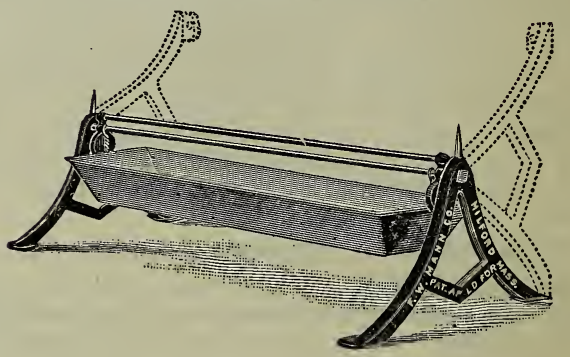

18-inch Tray . . . . . . . . . \$1.00

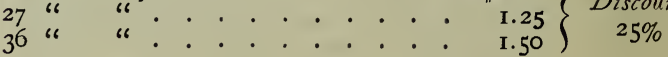

MANN'S CAST IRON MORTAR.

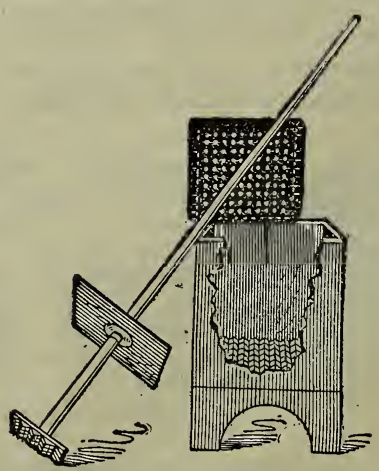

Designed expressly for breaking glass, crockery ware, oyster shells, or other dry substances for poultry. Each, $\$ 3.75$. Discount $20+10 \%$. 
POULTRY SUPPLIES.-Continued.

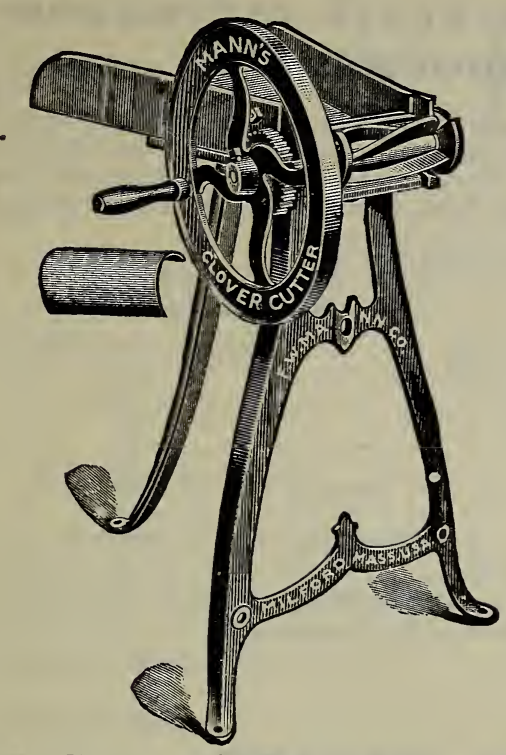

Cutter, Mann's, Clover. Don't buy a clover cutter until you have seen the Mann's. Made either with or without the stand.

Clover Cutter, no stand . . Price, each, $\$ 10.00$

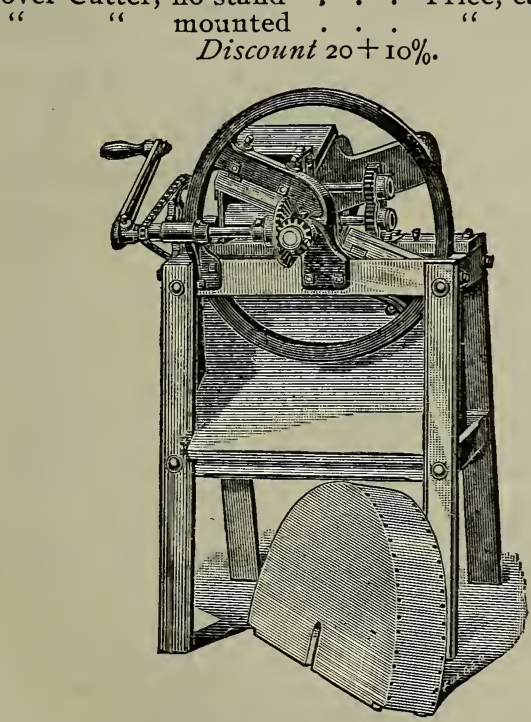

Cutter, Clover, The Ross. The latest and best green clover cutter for the poultryman.

Mfrs. price, each . . \$20.00. Discount $40 \%$.

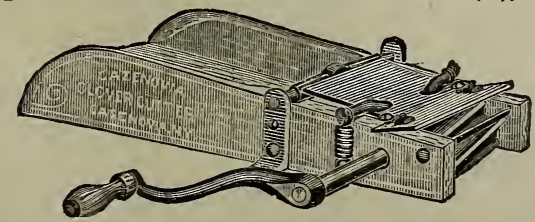

Cutter, Clover, Webster. For small flocks this has no equal. It is well made and is not a toy, but intended for actual service.

Price, each . . . . . . . . . . . . $\$ 2.75$
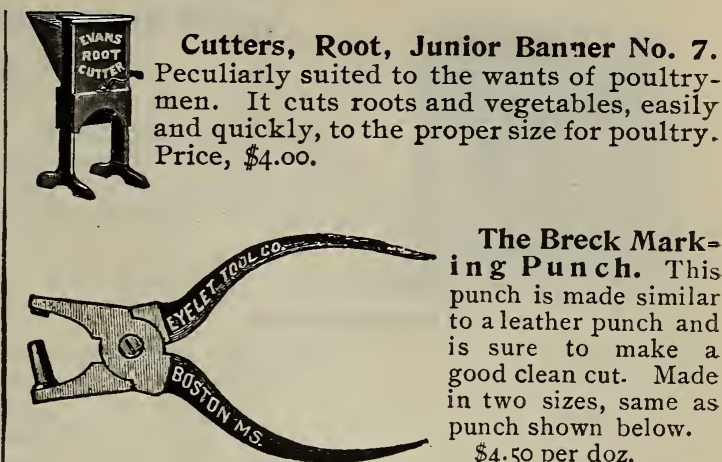

The Breck Mark= in $\mathbf{g} \mathbf{P u} \mathbf{n} \mathbf{c h}$. This punch is made similar to a leather punch and is sure to make a good clean cut. Made in two sizes, same as punch shown below.

$\$ 4.50$ per doz.

This little instrument is for marking young and old chickens, and all kinds of fowls, and is simple, cheap and effective. Made in two sizes, as shown in cut at the right. Price, $\$ 2.25$ per doz.
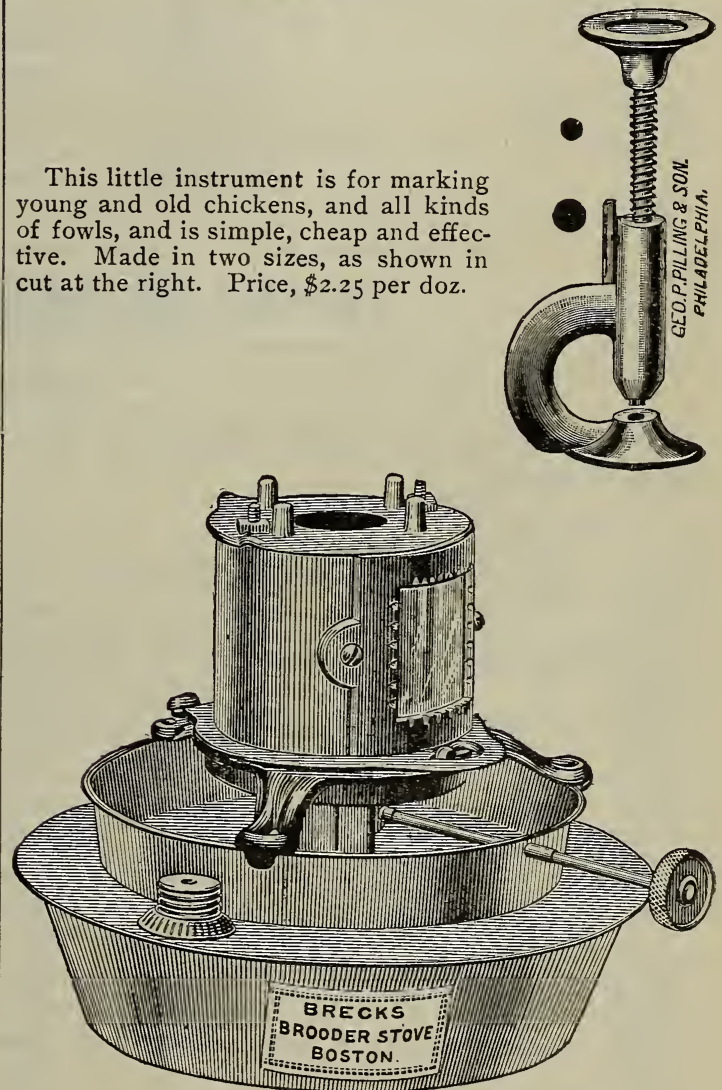

Stoves, Brooder. A really superior, effective and safe stove. The oil fount is pressed out of one piece sheet iron and then heavily galvanized, so that leaking is impossible. \$Ir.oo per doz.

Cookers, Granite State Feed.

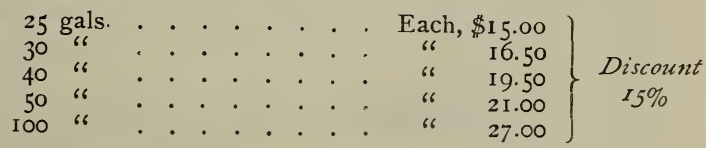

Truck, Water Barrel.

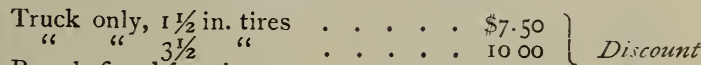

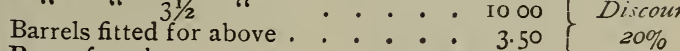

Boxes for above . . . : : $\begin{aligned} & 3.50 \\ & 350\end{aligned}$ 


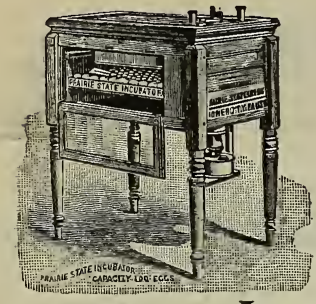

Incubators.

No. I Baby<smiles>C#CC#CC#CC#C</smiles>

Special Duck

No. I Special "6 2 66

Discount ro\%.

\section{GENUINE PRAIRIE STATE INCUBATORS AND BROODERS.}

The least that can be said of these famous: incubators is "they are without equal." The U. S. government uses them exclusively at the Experiment Stations at Washington, D. C. The largest poultry breeders in the country also use them exclusively, and they are the standard from which all incubators are judged.

Price on application.

\section{Brooders.}

No. I Style A . . Capacity roo Chicks, \$I3 50

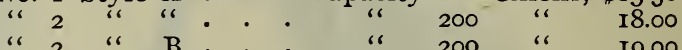

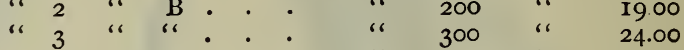

" 4 " " " 4 " . . .

" I " C Outdoor " "

" 2 " " " "

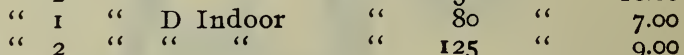

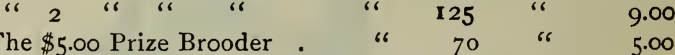

Discount ro $\%$, excepting Style B, which are $5 \%$

PRICES OF EGGS AND POULTRY.

Prices quoted are net and subject to change.

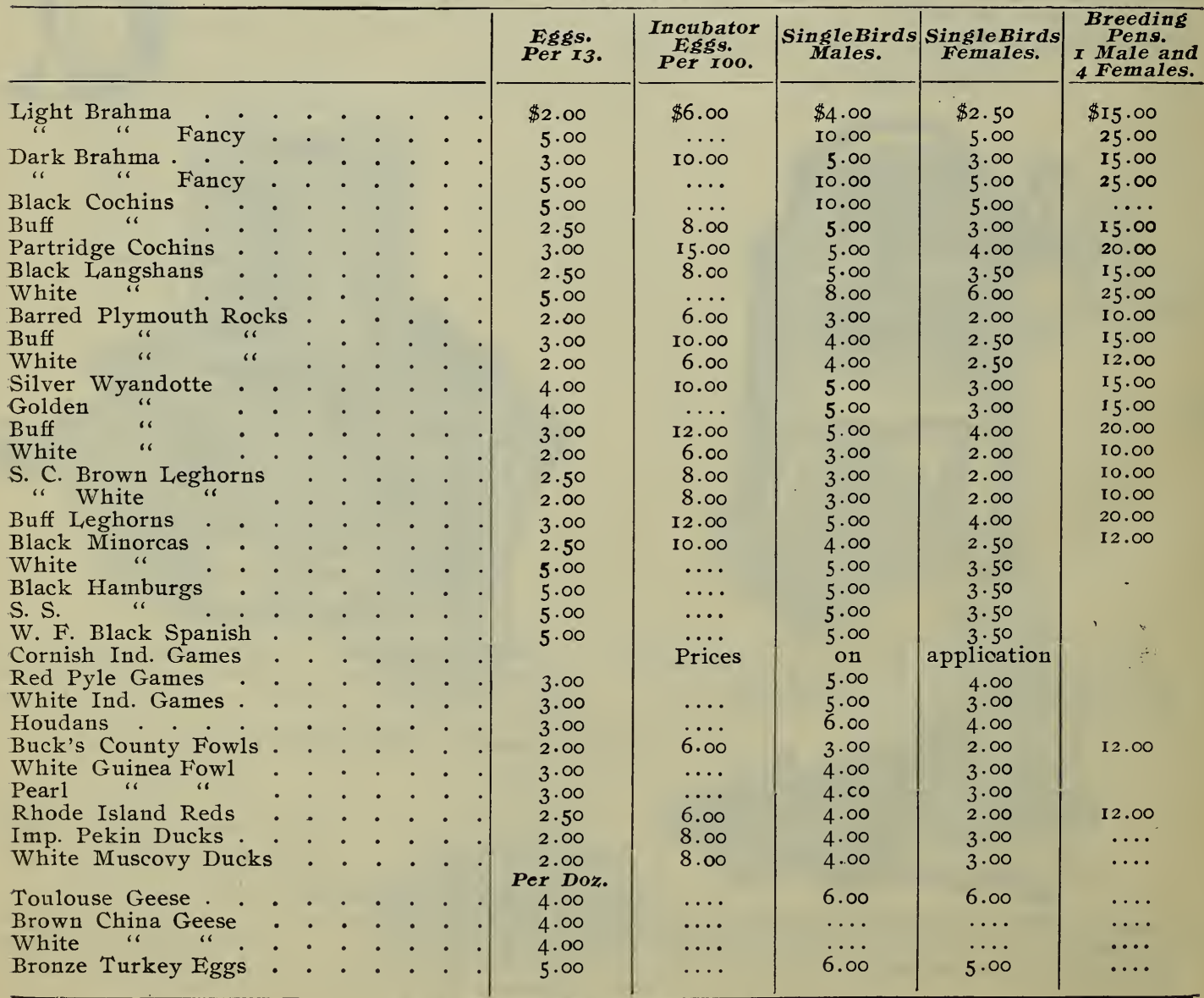




\section{BOOKS ON RURAL TOPICS.}

\section{ALL BOOKS DELIVERED FREE AT PRICES QUOTED.}

Discount $20 \%$

\section{GARDENING.}

Dictionary of Gardening. Edited by GEORGE Nicholsox. 4 vols. 2000 illustrations........\$20.00 Dictionary, Johnson's Gardeners'. By WRIGHT \& Dewar.......................... 4.00 Bulbs and Tuberous Rooted Plants. By C. H. Allen ................................ 1.50 Lilies and Their Culture. By Dr. WALlaCE.. 1.75 Hand Book of Plants. By Peter Hexdersox.. 4.00 Gardening for Pleasure. By "6 "6 . 1.50 Water Garden, The. By WM. Tricker...... 2.00 Garden Making. By Prof. Bailex.......... 1.00 Cut Flowers and How to Grow. By M. A. Huxt 2.00 Practical Floriculture. By Peter Hexdenson 1.50 Success with House Plants. By L. P. HillHouse..............Paper cover, 50 cts.; cloth, 1.00 Residential Sites and Environments. By J. F. Johxsox............................... 2.50

Violet Culture (Commercial). By Prof. GaLLoway ............................ 1.50

Rose, Parsons on the. By S. B. Parsors..... 1.00 Plant Breeding. By Prof. Bailex......... 1.00 Nursery Book. " " " " " " $\quad$ " $\quad \ldots \ldots \ldots \ldots 1.00$ Pruning Book. " " " " " Survival of the Unlike, The. By Prof. Balley 2.00 Forcing Book. Cultivation of Vegetables under Glass. By Prof. Bailey................ 1.00

Gardening for Profit. By Peter Hexdersox.. 1.50 Success in Market Gardening. By W. W. RAwsox ............................. 1.00 Asparagus Culture. By Barses \& Robinsox.. .50 Celery Culture for Profit. By T. Greiner.... .20 Celery, How to Grow Anywhere. By P. Schen . .50 Mushrooms, How to Grow Them. By W. FALCONer,............................... 1.50 Mushroom Culture. By W. RoBissox....... .50

\section{FARMING.}

American Farm Book. By R. L. \& L. F. Allex 2.00 How the Farm Pays. By Hexdersox \& Cro-

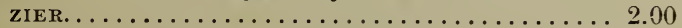

Forage Crops, other than Grasses. By Prof. Thos. Shaw ......................... 1.00

Grasses and Clover, Field Roots. By Prof.

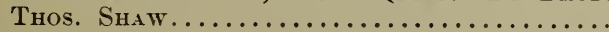

Grasses and Forage Plants. By ChAs. L. Flint $\$ 2.00$ Silos and Ensilage. By Maxu Miles....... .50 Chemistry of the Farm. By R. Warringtox. 1.00 Soil, The. By F. H. Kixg.............. .75 Manures, Talks on. By JosePH Harris, M.S. 1.50 Fertilizers. By Prof. Voorhees.......... 1.00 Drainage, Tile. By W. J. Сhaмberlaix....... .35 Irrigation for Farm, Garden and Orchard. By

Hexry Stewart......................... 1.00 Allen's American Cattle. By L. F. Allex... 2. 2.50 Pig, Harris on the. By Joseph Harris...... 1.00 Feeding Animals. By E. W. Stewart...... 2.00 Dairyman's Manual. By Hexry Stewart.... 1.50 Milk and its Products. By Prof. H. H. WIxg. 1.00

\section{FRUIT.}

Fruit Growing, The Principles of. By Prof.

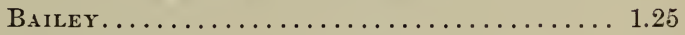

Fruit Garden, The. By P. BarRy........ 1.50

Bush Fruits. By Prof. Card........... 1.50

Berry Book, The Biggle............... .50

Fruits and Fruit Trees of America. By A. J.

Dowsixg..................... 5.00

Fruit Grower, The Practical. By C. T. MAY-

NARD......................... 50 Small Fruit Culturist. By A. S. Fuller..... 1.00 Cranberry Culture. BУ J. J. Whiте........ 1.00 Cape Cod Cranberries. By JAs. Weвв....... .40 Pear Culture for Profit. By P. T. Quiss..... 1.00 Strawberry Culturist. By A. S. Fuller..... .25 Strawber ry Culture, The A B C of. By T. B.

Terry.......................... 40

\section{FORESTRY.}

Practical Forestry. By A. S. Fuller........ 1.50 Outlines of Forestry. By E. J. Houstow, A.M. 1.00 Trees for Street and Shade. By Powell \&

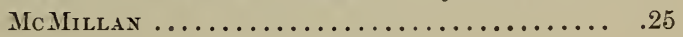

\section{INSECTS AND SPRAYING.}

Injurious Insects of Farm and Garden. By Mrs. M. Treat.......................... 1.50 Spraying of Plants, The. By Prof. Lodemax. 1.00 


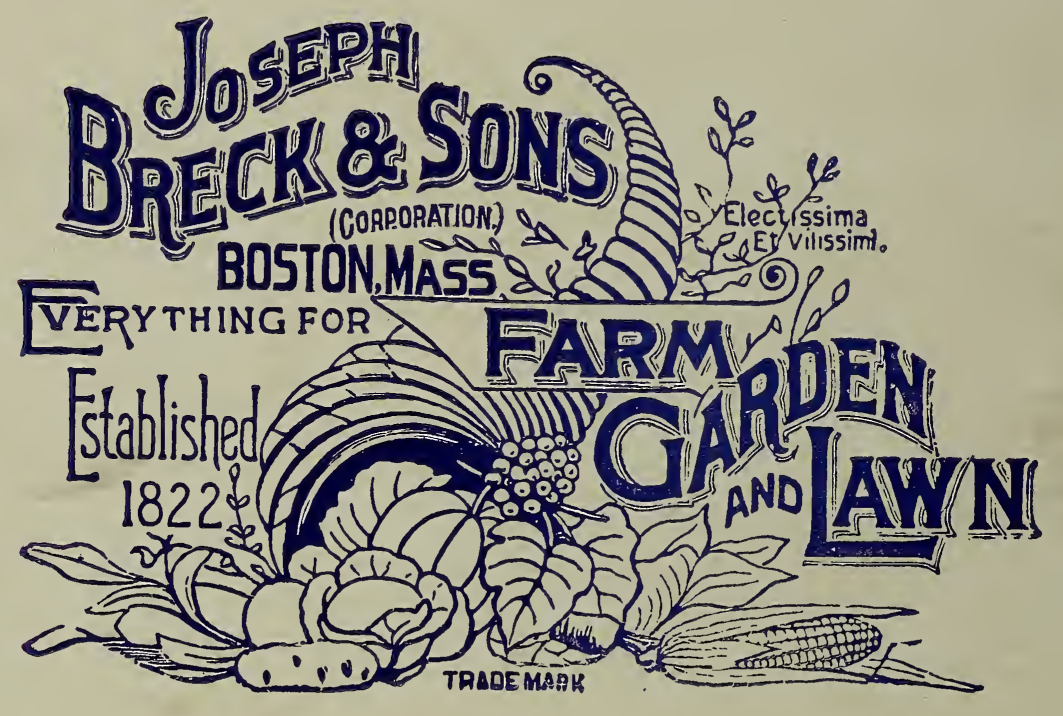

